*ak RMIS View/Frint Document Cover Sheet tow

This document was retrieved from the Documentation and Records Manaqement (DRM) ISEARCH System. It is intended for Information only and may not be the most recent or updated version. Contact a Document Service Center (see Hanford Info for locations) if you need additional retrieval information.

Accession \#: D196069136

Document \#: SD-NR-DB-004

Title/Desc:

END POINT CRITERIA FOR FUEL SUPPLY SHUTDOWN FACILITIES

Pages: 109 
3. From: (Originating Organization) Fuel Supply Shutdown

5. Proj./Prog./Dept./Div.:

Fuel Supply Shutdown/FFFTP/TRP

\section{Originator Renarks: \\ Original issue - Revision 0}

6. Cog. Engr.:

John A. Remaize W/9800 K33A2

\section{EDT 606213}

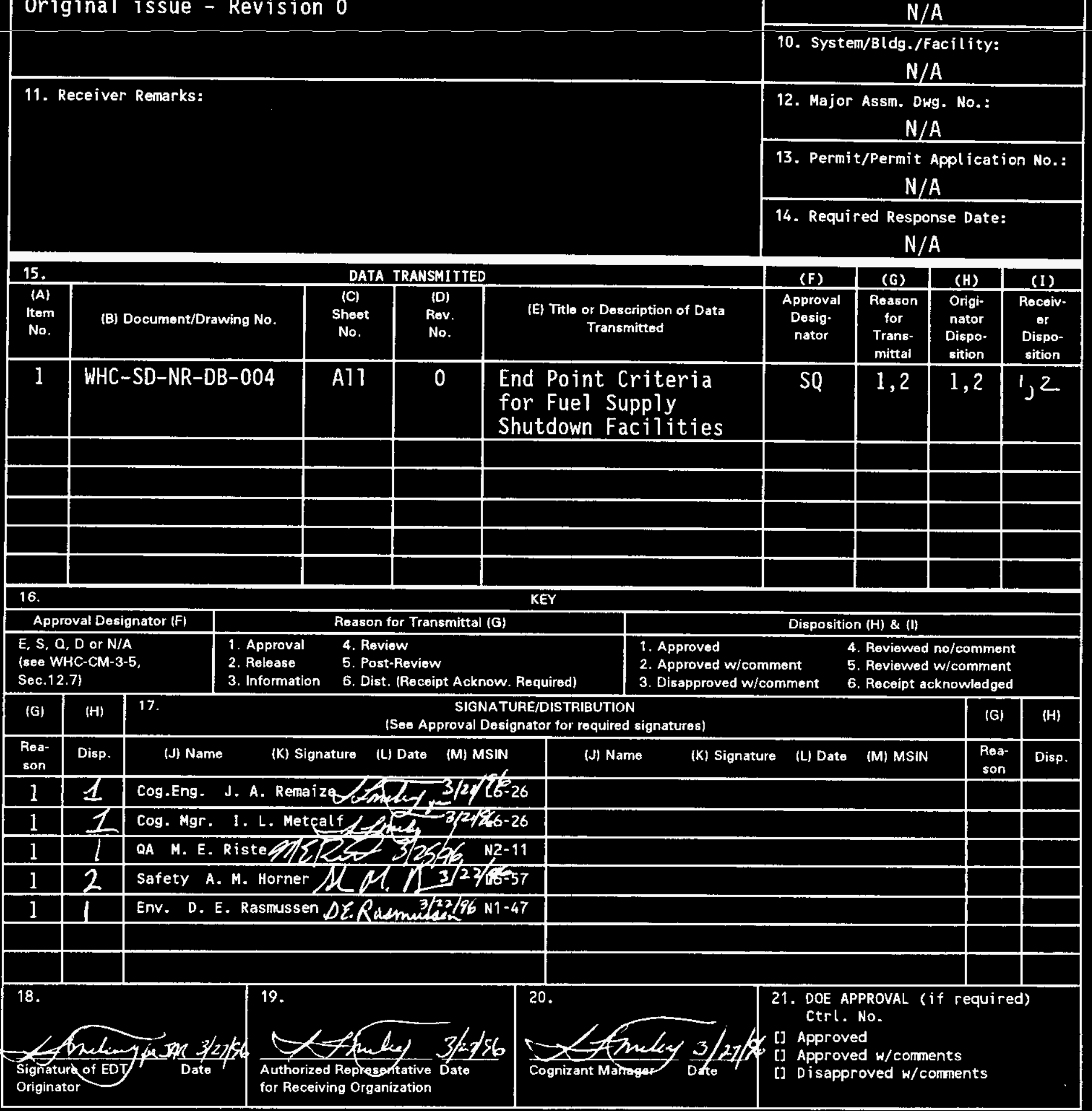




\title{
End Point Criteria for Fuel Supply Shutdown Facilities
}

\author{
J. E. Remaize \\ West inghouse Hanford Company, Richland, WA 99352 \\ U.S. Department of Energy Contract DE-AC06-87RL10930 \\ 606213 \\ EDT/ECN: 600310 \\ Org Code: 19800 \\ UC: UC-2050 \\ B\&R Code: EW7003000 \\ Charge Code: K33A2 \\ Total Pages: 104106
}

Key Words: End Point Criteria, D\&D, ERC, Fuel Supply Shutdown

Abstract: This document covers the End Point Criteria for Fuel Supply Shutdown Facilities that is to be attained for the transfer of these facilities to the EM-40 Program.

TRADEMARK DISCLAIMER. Reference herein to any specific commercial product, process, or service by trade name, trademark, manufacturer, or otherwise, does not necessarily constitute or imply its endorsement, recomendation, or favoring by the United States Government or any agency thereof or its contractors or subcontractors.

Printed in the United States of Anerica. To obtain copies of this document, contact: WHC/BCS Document Control Services, P.O. Box 1970, Mailstop H6-08, Richland WA 99352, Phone (509) 372-2420; Fax (509) 376-4989.
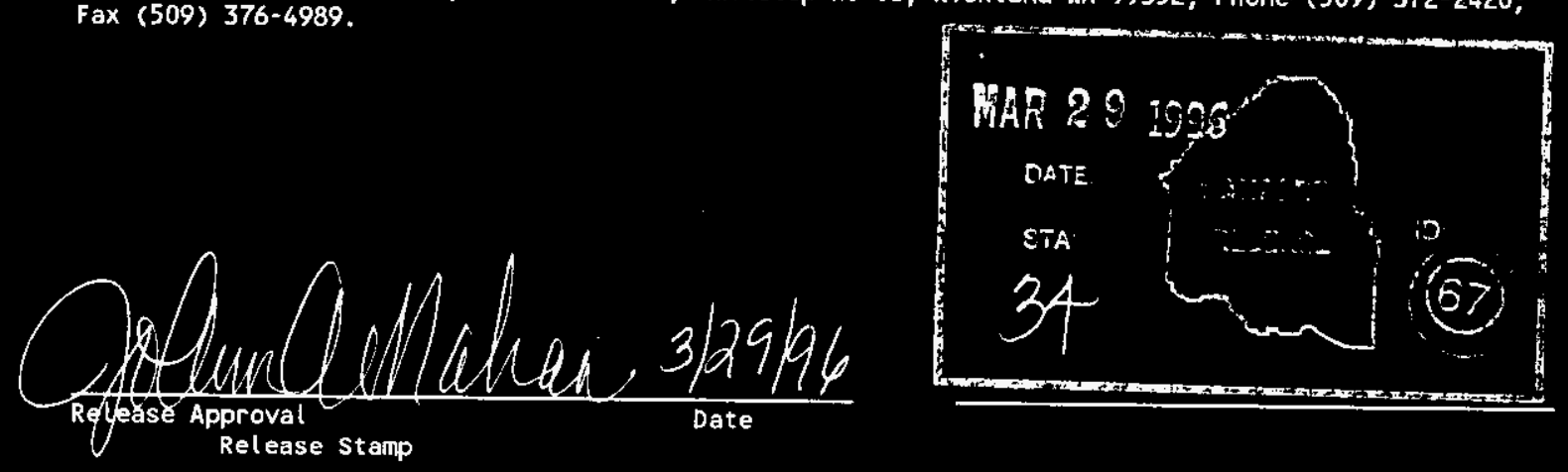

\section{Approved for Public Release}


TABLE OF CONTENTS

Page

$\begin{array}{ll}1.0 & \text { Introduction } \\ 2.0 & \text { Facility Buildings } \ldots \ldots \ldots \ldots \ldots \\ 3\end{array}$

3.0 Development of Criteria . . . . . . . . . . 1

4.0 Rain Water Drainage . . . . . . . . . . $2_{2}^{1}$

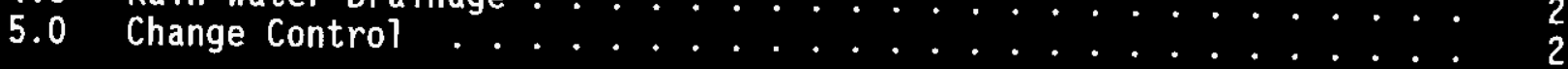

End Point Criteria - 333 .............. 5

End Point Criteria - 303A, B, E, and G . . . . . . 14

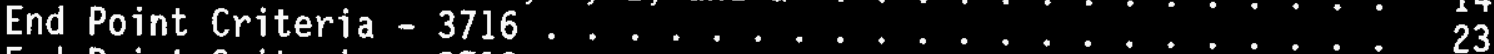

End Point Criteria - 3712 ................... 30

End Point Criteria - 313 South . . . . . . . . 37

End Point Criteria - 313 North . . . . . . . . . . 43

End Point Criteria - 304 .................. 49

End Point Criteria - 334, 334A . . . . . . . 55

End Point Criteria - M0052 Trailer . . . . . . . . . 62

End Point Criteria - 303M . . . . . . . . 68

End Point Criteria - 303K, 3707G . . . . . . . . 75

End Point Criteria - 303F . . . . . . . . . 81

End Point Criteria - 334 Tank Farm . . . . . . . . 87

End Point Criteria - 311 Tank Farm . . . . . . . 93

End Point Criteria - Outside Trench System . . . . . . 99

Administrative End Points ................ 104 
END POINT CRITERIA FOR FUEL SUPPLY SHUTDOWN FACILITIES

\subsection{INTRODUCTION}

This report documents the End Point Criteria for Fuel Supply Shutdown (FSS) facilities that has to be attained for the transfer of these facilities to the EM-40 Program.

\subsection{FACILITY - BUILDINGS}

The FSS includes the following buildings with noncontiguous boundaries: $313,333,303 \mathrm{~A}, 303 \mathrm{~B}, 303 \mathrm{E}, 303 \mathrm{~F}, 303 \mathrm{G}, 303 \mathrm{~K} / 3703 \mathrm{G}, 303 \mathrm{M}, 304,334,334 \mathrm{~A}$, 3712, 3716, and M0052. Also included are the 311 Tank Farms and the 334 Tank Farms. There is a trench system that contains piping interconnecting $334 \mathrm{TF}$, $334 \mathrm{~A}, 333,303 \mathrm{~F}, 313$, and $311 \mathrm{TF}$ that is addressed.

WHC-CM-1-3, Management Requirements and Procedures, Section 6.12, "Building Management," Rev. 2, Section 4.4, item 2, states: "Maintaining the area within 20 feet of the building structure outer 1 imits with respect to grounds cleanup and general housekeeping. This includes debris moved to areas outside the original area of responsibility. In cases where two buildings are less than 40 feet apart, the median point between the two buildings will be the division line." The preceding will establish the footprint of facilities to be transferred except that the $333,3716,334,334 \mathrm{~A}, 334 \mathrm{TF}$, and $303 \mathrm{M}$ footprint will include all areas within the surrounding fence.

\subsection{DEVELOPNENT OF CRITERIA}

Bechtel Hanford, Inc. (BHI) has provided FSS with a 1 ist of 40 requirements that have to be met for the trasnfer of the faiclities to the EM-40 Program.

Fuel Supply Shutdown determined what requirements were applicable to each building and how these requirements will be met. Several building walkdowns were conducted with BHI and FSS personnel to assure understanding of the requiremetns and actions.

The attached End Point Acceptance Criteria Checklist for the FSS buildings documents the process and, when approved, will be the basis for the continuing efforts of transition for FSS.

The Administrative End Points Checklist covers the administrative documentation issues not included in the End Point Acceptance Criteria Checklist. 
As the various transition activities are completed, the implementation/ verification document will be inserted in the appropriate column.

\subsection{RAIN WATER DRAINAGE}

There are no provisions to compensate for rain water disposal. The rain collection drains to the process sewer will not be plugged. However, the rain water collected in the Tank Farms berm will be allowed to accumulate and evaporate.

\subsection{CHANGE CONTROL}

Changes to End Point Acceptance Criteria Checklist can be made when warranted by preparing an engineering change notice and having it approved by the same level of BHI and Westinghouse Hanford Company approval management. 
END POINT CRITERIA TABLES 
This page intentionally left blank 


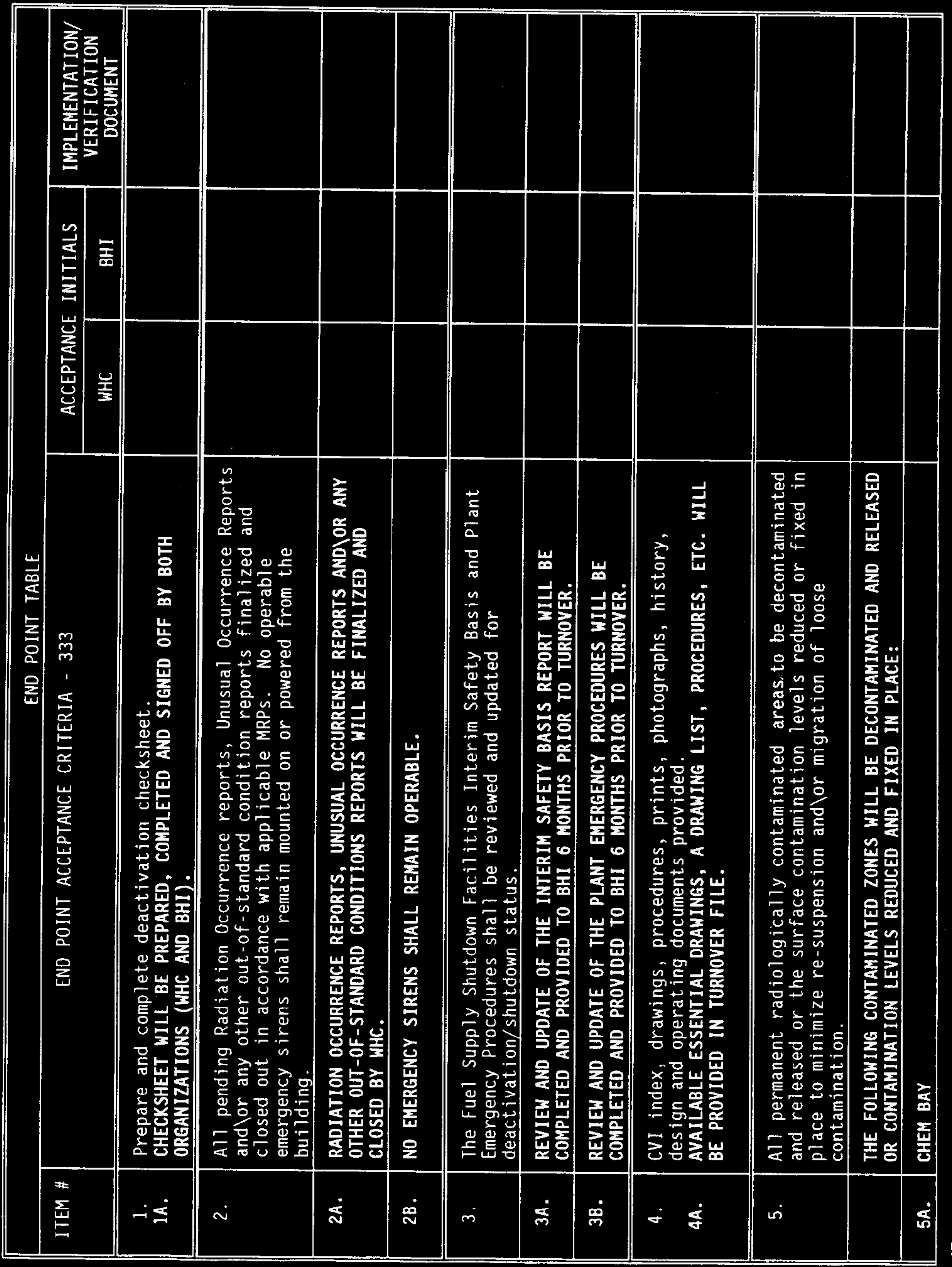




\begin{tabular}{|c|c|c|c|c|}
\hline \multicolumn{5}{|c|}{ END POINT TABLE } \\
\hline \multirow[t]{2}{*}{ ITEM \# } & \multirow[t]{2}{*}{ END POINT ACCEPTANCE CRITERIA - 333} & \multicolumn{2}{|c|}{ ACCEPTANCE INITIALS } & \multirow{2}{*}{$\begin{array}{l}\text { IMPLEMENTATION/ } \\
\text { VERIFICATION } \\
\text { DOCUMENT }\end{array}$} \\
\hline & & WHC & BHI & \\
\hline $5 \mathrm{~B}$. & CHEM BAY MEZZANINE & & & \\
\hline $5 C$. & BILLET CLEANING AREA & & & \\
\hline 50. & $\begin{array}{l}\text { THERE WILL BE NO DECONTAMINATION OR STABILIZATION DONE TO THE CUT UP } \\
\text { AREA }\end{array}$ & & & \\
\hline 6 . & $\begin{array}{l}\text { Full compliance with Hanford Site Radiological Control Manual must } \\
\text { be assured, especially as it pertains to radiological posting. } \\
\text { COMPLIANCE WITH HANFORD SITE RADIOLOGICAL CONTROL MANUAL WILL BE } \\
\text { CONFIRMED BY HAVING 300 AREA RADIOLOGICAL CONTROL PERFORM AN } \\
\text { ASSESSMENT AND WHC WILL CORREC ALL NOTED DISCREPANCIES. }\end{array}$ & & & \\
\hline 7 . & $\begin{array}{l}\text { Temporary radiologically posted areas are cleaned up and } \\
\text { radiologically released. } \\
\text { TENPORARY RADIOLOGICALLY POSTED AREAS WILL BE CLEANED UP AND } \\
\text { RELEASED. }\end{array}$ & & & \\
\hline 8. & $\begin{array}{l}\text { All stored radioactive and mixed waste removed. } \\
\text { RADIOACTIVE AND MIXED WASTE REMOVED. }\end{array}$ & & & \\
\hline 9. & $\begin{array}{l}\text { A11 hazardous materials used for deactivation and cleanup work must } \\
\text { be collected and disposed of in accordance with requirements. } \\
\text { ALL HAZARDOUS MATERIALS USED FOR DEACTIVATION AND CLEANUP WILL BE } \\
\text { REMOVED. }\end{array}$ & & & \\
\hline 10. & $\begin{array}{l}\text { All unattached hazardous materials (i.e., loose friable asbestos, } \\
\text { lead. mercury, etc.) must be removed from the facility and disposed } \\
\text { of in accordance with requirements. } \\
\text { ALL UNATTACHED HAZARDOUS HATERIALS WILL BE REMOVED. }\end{array}$ & & & \\
\hline 11. & $\begin{array}{l}\text { Attached hazardous materials located, identified, quantified, } \\
\text { labelled as necessary, and recorded. }\end{array}$ & & & \\
\hline $11 \mathrm{~A}$. & $\begin{array}{l}\text { ATTACHED HAZARDOUS MATERIALS WILL BE LOCATED, IDENTIFIED, } \\
\text { QUANTIFIED, LABELLED AS NECESSARY AND RECORDED. SOME SPECIFIC } \\
\text { ACTIONS ARE NOTED BELOW. }\end{array}$ & & & \\
\hline
\end{tabular}




\begin{tabular}{|c|c|c|c|c|}
\hline \multicolumn{5}{|c|}{ END POINT TABLE } \\
\hline \multirow[t]{2}{*}{ ITEM \#. } & \multirow{2}{*}{ END POINT ACCEPTANCE CRITERIA - 333} & \multicolumn{2}{|c|}{ ACCEPTANCE INITIALS } & \multirow{2}{*}{$\begin{array}{l}\text { IMPLEMENTATION/ } \\
\text { VERIFICATION } \\
\text { DOCUMENT }\end{array}$} \\
\hline & & WHC & BHI & \\
\hline 118. & $\begin{array}{l}\text { BERYLLIUM SYSTEMS WILL BE DECONTAMINATED AND RELEASED WHERE } \\
\text { POSSIBLE. SAMPLES WILL BE ANALYZED AND AREAS WHERE DECONTANINATION } \\
\text { WAS NOT SUCCESSFUL WILL BE CONTAINED OR STABILIZED AND DOCUNENTED AS } \\
\text { REQUIRED. }\end{array}$ & & & \\
\hline 11C. & VACCU-BLAST DUST COLLECTOR WILL HAVE DUST COLLECTION MEDIA REMOVED. & & & \\
\hline 110. & $\begin{array}{l}\text { CHEM BAY EXHAUST SYSTEM WILL BE DECONTAMINATED AND RELEASED WHERE } \\
\text { POSSIBLE. SAMPLES WILL BE ANALYZED AND AREAS WHERE DECONTAMINATION } \\
\text { WAS NOT SUCCESSFUL WILL BE DOCUMENTED AS REQUIRED. }\end{array}$ & & & \\
\hline $11 \mathrm{E}$. & $\begin{array}{l}\text { WATS SYSTEM COMPONENTS WILL BE CLEAN CLOSED ACCORDING TO RCRA } \\
\text { CLOSURE PLAN. }\end{array}$ & & & \\
\hline $11 \mathrm{~F}$. & CHEM BAY PIPING WILL BE REMOVED FROM THE TRENCHES. & & & \\
\hline $\begin{array}{l}12 . \\
12 \mathrm{~A} .\end{array}$ & $\begin{array}{l}\text { All reactor fuel elements and } \backslash \text { or other source and special materials } \\
\text { must be removed from the building. } \\
\text { ALL SPECIAL NUCLEAR MATERIAL WILL BE REMOVED FROM THE BUILDING. }\end{array}$ & & & \\
\hline $\begin{array}{l}13 . \\
13 A .\end{array}$ & $\begin{array}{l}\text { Final radiological status surveys available. } \\
\text { FINAL RADIOLOGICAL SURVEYS WILL BE INCLUDED WITH THE TURNOVER } \\
\text { PACKAGE. }\end{array}$ & & & \\
\hline 14. & Tanks, vessels, drums, etc. drained and heels removed. & & & \\
\hline & $\begin{array}{l}\text { ALL TANKS, VESSELS, DRUMS, ETC. WILL BE DRAINED AND HEELS REMOVED. } \\
\text { TANKS WILL BE SWIPE SAMPLED AND CHECKED FOR PH VALUE: }\end{array}$ & & & \\
\hline $14 \mathrm{~A}$. & WATS SYSTEM TANKS -2 TANKS & & & \\
\hline 14B. & COMPONENT CLEANING LINE - 6 TANKS. & & & \\
\hline $14 c$. & BILLET CLEANING LINE -6 TANKS. & & & \\
\hline 14D. & FINAL ETCH CLEANING LINE -5 TANKS. & & & \\
\hline 14E. & URANIUM RECOVERY SYSTEM - 3 TANKS. (CHEM BAY AREA) & & & i. \\
\hline 14F. & PRE-NELD ETCH CLEANING LINE -7 TANKS. & & & \\
\hline 14G. & COPPER STRIP CLEANING LINE -4 TANKS. & & & \\
\hline $14 \mathrm{H}$. & PRE-BRAZE ETCH CLEANING LINE -6 TANKS. & & & \\
\hline
\end{tabular}




\begin{tabular}{|c|c|c|c|c|}
\hline \multicolumn{2}{|r|}{ END POINT TABLE } & \multicolumn{3}{|c|}{$\cdot$} \\
\hline \multirow[t]{2}{*}{ ITEM \# } & \multirow[t]{2}{*}{ END POINT ACCEPTANCE CRITERIA - 333} & \multicolumn{2}{|c|}{ ACCEPTANCE INITIALS } & \multirow{2}{*}{$\begin{array}{l}\text { IMPLEMENTATION/ } \\
\text { VERIFICATION } \\
\text { DOCUMENT }\end{array}$} \\
\hline & & WHC & BHI & \\
\hline $14 \mathrm{I}$. & AUTOCLAVE AREA - 16 VESSELS. & & & \\
\hline $14 \mathrm{~J}$ & URANIUM RECOVERY SYSTEM - 3 TANKS. (OUTDOOR) & & & \\
\hline $14 K$. & WATER TREATMENT SYSTEM - 11 TANKS OR VESSELS. & & & \\
\hline $14 \mathrm{~L}$. & CHEM SYSTEM SCRUBBER - 1 TANK. & & & $\cdot$ \\
\hline $14 M$. & URANIUM SCRUBBER SYSTEM -1 TANK. & & & \\
\hline 15. & $\begin{array}{l}\text { All elevator and crane systems are laid up with documentation of the } \\
\text { type, weight and class of fluid required for operation, should that } \\
\text { become desirable in the future. }\end{array}$ & & & \\
\hline $15 A$. & THERE ARE NO ELEVATORS IN THIS BUILDING. & & & \\
\hline $15 B$ & $\begin{array}{l}\text { CRANES AND HOISTS WILL NOT BE DRAINED. AVAILABLE REQUIRED } \\
\text { INFORMATION WILL BE SUPPLIED FOR THESE CRANES AND HOISTS: }\end{array}$ & & & \\
\hline $15 B .1$ & 5-TON AUTOCLAVE CRANE & & & \\
\hline 158.2 & 10-TON PRESS CRANE & & & \\
\hline $15 \mathrm{~B} .3$ & CHEM BAY 8 HOISTS. & & & \\
\hline $15 B .4$ & COMPONENT CLEANING LINE HOIST. & & & \\
\hline 16. & $\begin{array}{l}\text { Documentation is available confirming that zero energy checks were } \\
\text { made on all de-energized electrical circuits and isolated } \\
\text { pressurized systems e.g., water service air, steam, etc. using } \\
\text { existing documents to the extent possible. }\end{array}$ & & & \\
\hline $16 A$. & $\begin{array}{l}\text { WATER, STEAM, AND SERVICE AIR WILL BE BLANKED AT LOCATION OUTSIDE } \\
\text { BUILDING. }\end{array}$ & & & \\
\hline $16 \mathrm{~B}$ & $\begin{array}{l}\text { IF ELECTRICAL SERVICE IS NOT DISCONNECTED BY CUTTING THE FEED } \\
\text { CABLES, ENERGY STATUS WILL BE DOCUMENTED ON ENERGIZED ELECTRICAL } \\
\text { SYSTEMS. }\end{array}$ & & & \\
\hline 17 . & $\begin{array}{l}\text { Electrical systems reduced to that necessary for S\&M and subsequent } \\
\text { D\&D. This includes removal of emergency light fixtures and the } \\
\text { associated batteries. Where appropriate, centralize the remaining } \\
\text { electrical services to a single point. }\end{array}$ & & & \\
\hline
\end{tabular}




\begin{tabular}{|c|c|c|c|c|}
\hline & & $\begin{array}{l}379 y 9 I 7 d d y \\
10 \mathrm{~N}\end{array}$ & 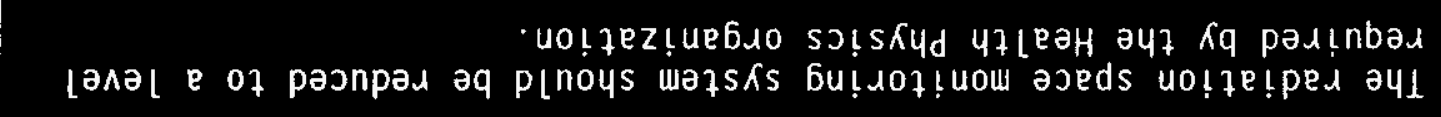 & I \\
\hline & & & 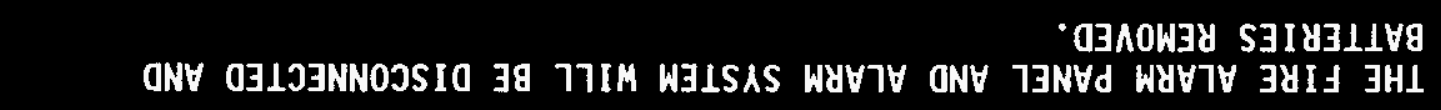 & $\cdot 202$ \\
\hline ' & & & 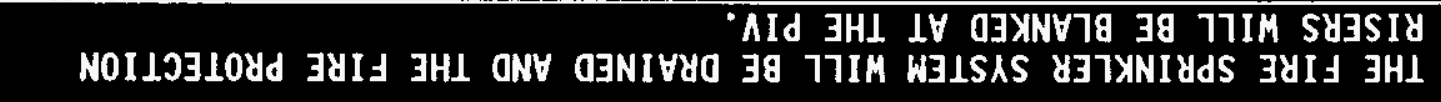 & $\cdot 802$ \\
\hline & & & 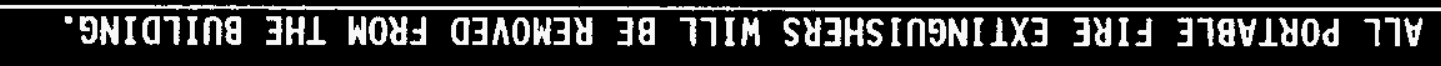 & - \\
\hline & & & рарелбимор süzsks uo!fjäold al!j & 02 \\
\hline & & & 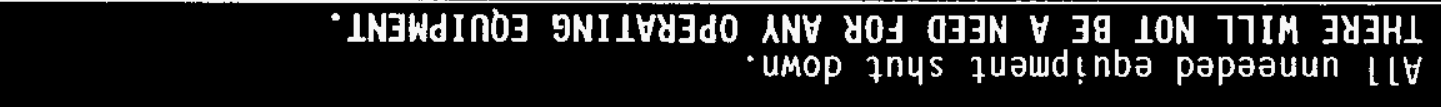 & $\begin{array}{l}\cdot 8 \mathrm{I} \\
\cdot 6 \mathrm{I}\end{array}$ \\
\hline & & & 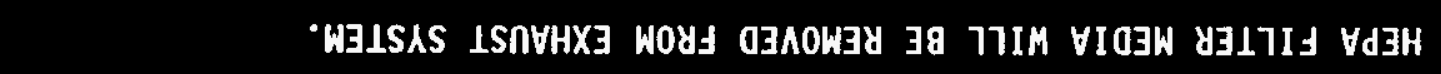 & $\cdot 381$ \\
\hline & & & 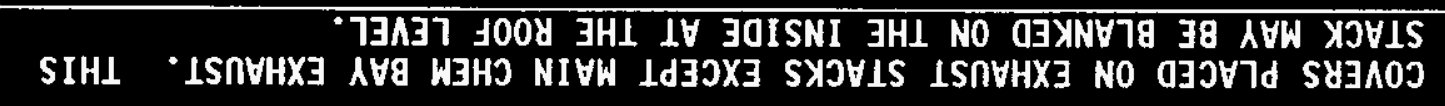 & $\cdot 08 \mathrm{I}$ \\
\hline & & & 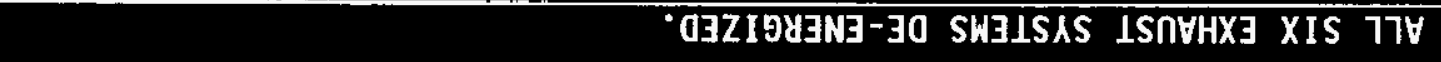 & $\cdot 98 \mathrm{I}$ \\
\hline & & & 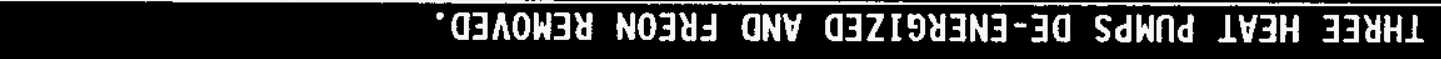 & $\cdot 881$ \\
\hline & & & 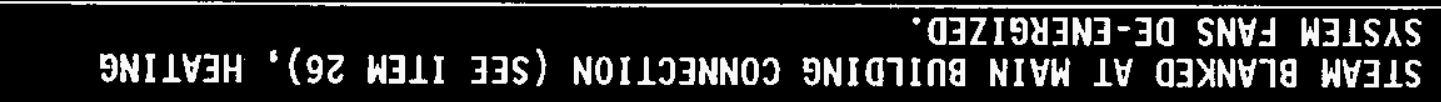 & $\cdot \forall 8 I$ \\
\hline & & & 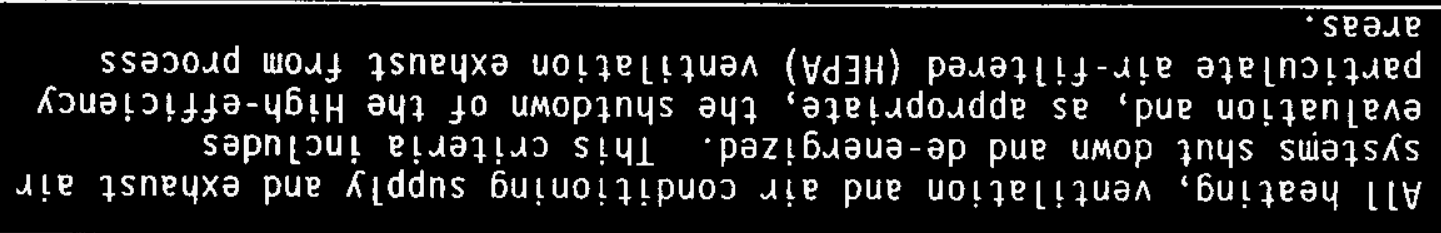 & $\cdot 81$ \\
\hline & & & 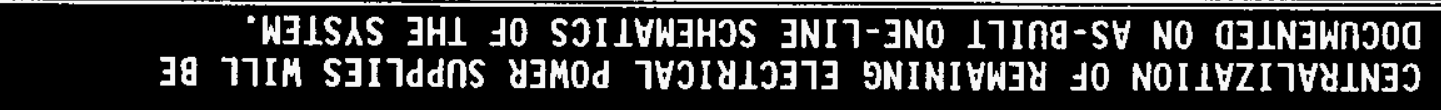 & $\cdot 8 \angle I$ \\
\hline & & & 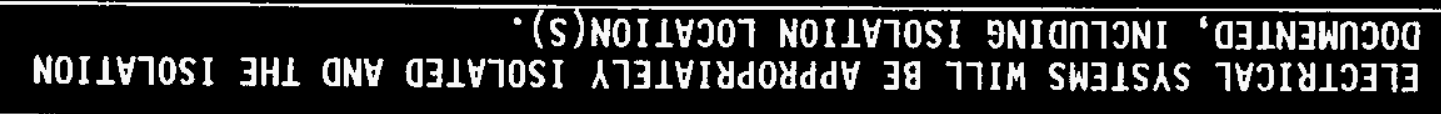 & $\cdot \forall \angle I$ \\
\hline $\begin{array}{c}\text { INJWกJO0 } \\
\text { NOI } \perp \forall J I\lrcorner I 8 \exists \Lambda\end{array}$ & IH马 & כHM & & \\
\hline /NOIIVINJWJ7dWI & \multicolumn{2}{|c|}{ 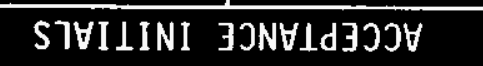 } & $\varepsilon \varepsilon \varepsilon-\forall I Y \exists \perp I Y J 3 J N \forall I d \exists J J \forall$ INIOd ONJ & $\# W \exists \perp I$ \\
\hline
\end{tabular}




\begin{tabular}{|c|c|c|c|c|}
\hline & & & 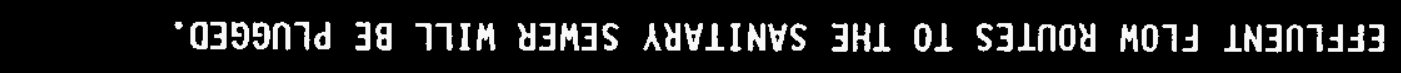 & $\cdot A L Z$ \\
\hline & & & NIV8O HONJYL 3dId AV8 KJHJ JOIS LSJM & $3 \angle 2$ \\
\hline & & & WOOY JYNH NI SNIVYO Y007] & $0 \angle 2$ \\
\hline & & & dWIS 8 & $J L 2$ \\
\hline & & & NIVYO HJKGYI JdId AVG WJHJ JOIS ISVI & $8 \angle 2$ \\
\hline & & & NIVYO VJaY dn IIIJ & ' $\mathbf{V} L \mathbf{Z}$ \\
\hline & & & 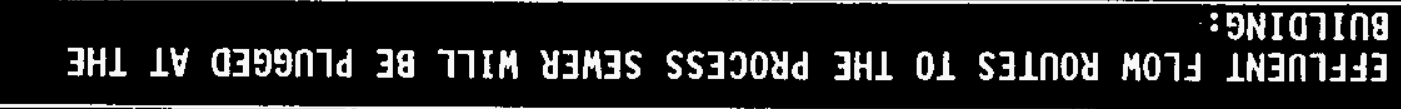 & \\
\hline & & & 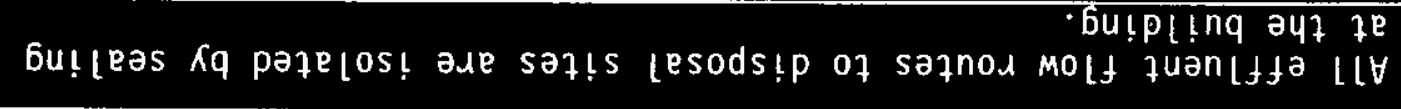 & $\angle 2$ \\
\hline & & & 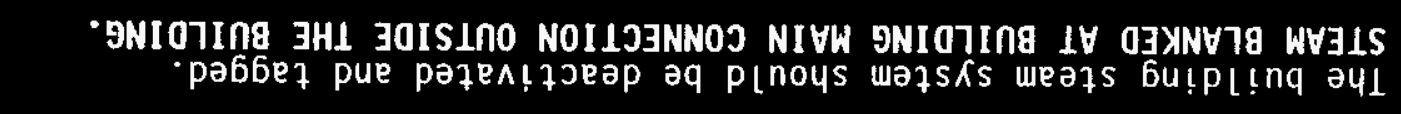 & $\cdot 992$ \\
\hline & & & 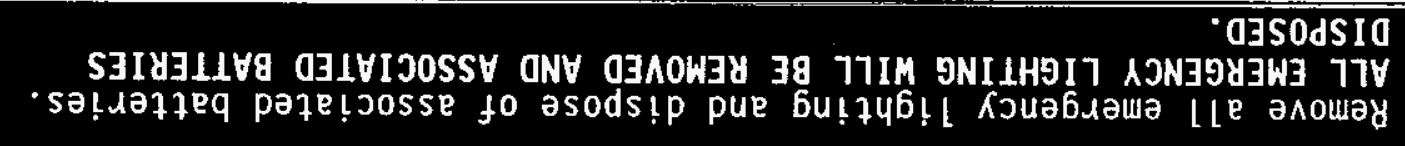 & 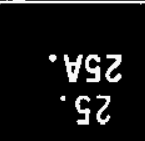 \\
\hline & & & 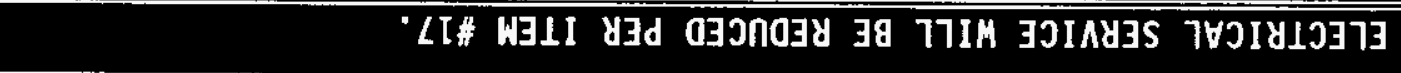 & $\cdot 362$ \\
\hline & & & 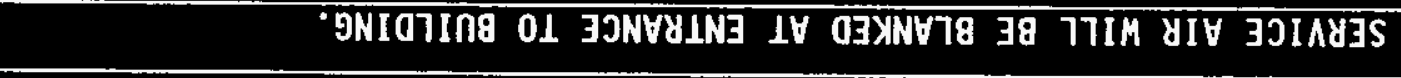 & 862 \\
\hline & & & 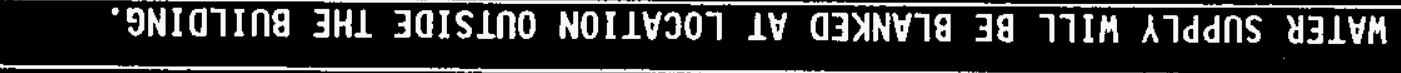 & $\forall 6 Z$ \\
\hline & & & 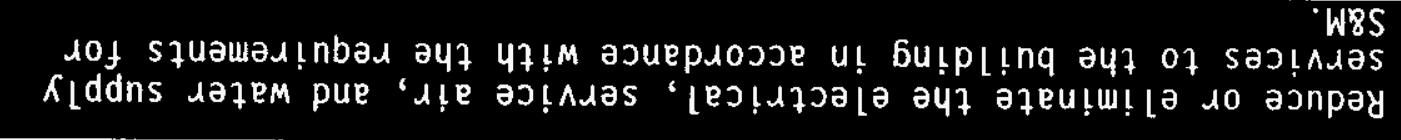 & $b 2$ \\
\hline & & & 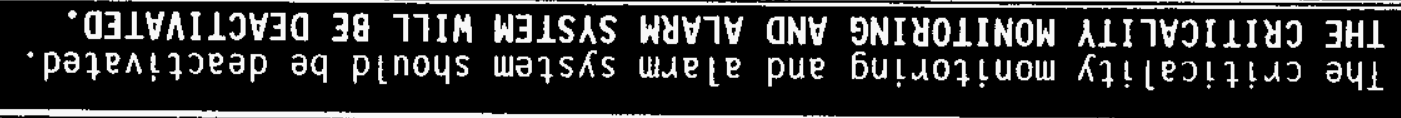 & ' \\
\hline & & $\begin{array}{l}\text { 378v9I7 } \\
10 \mathrm{~N} d \mathrm{~N}\end{array}$ & 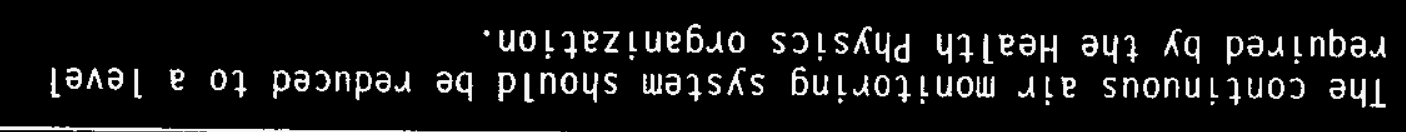 & $\cdot 22$ \\
\hline \multirow{2}{*}{ 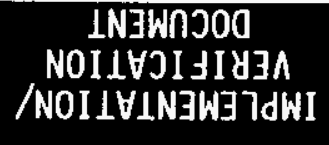 } & IH8 & כHM & \multirow[b]{2}{*}{ 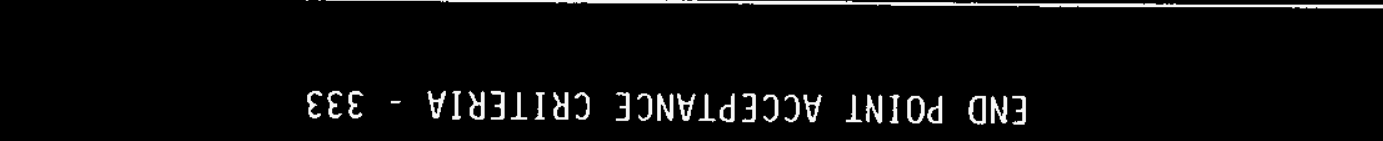 } & \multirow[b]{2}{*}{ \# WIII } \\
\hline & STVILINI & JכNVIdヨJכV & & \\
\hline
\end{tabular}




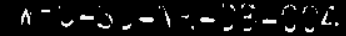

YEV. C

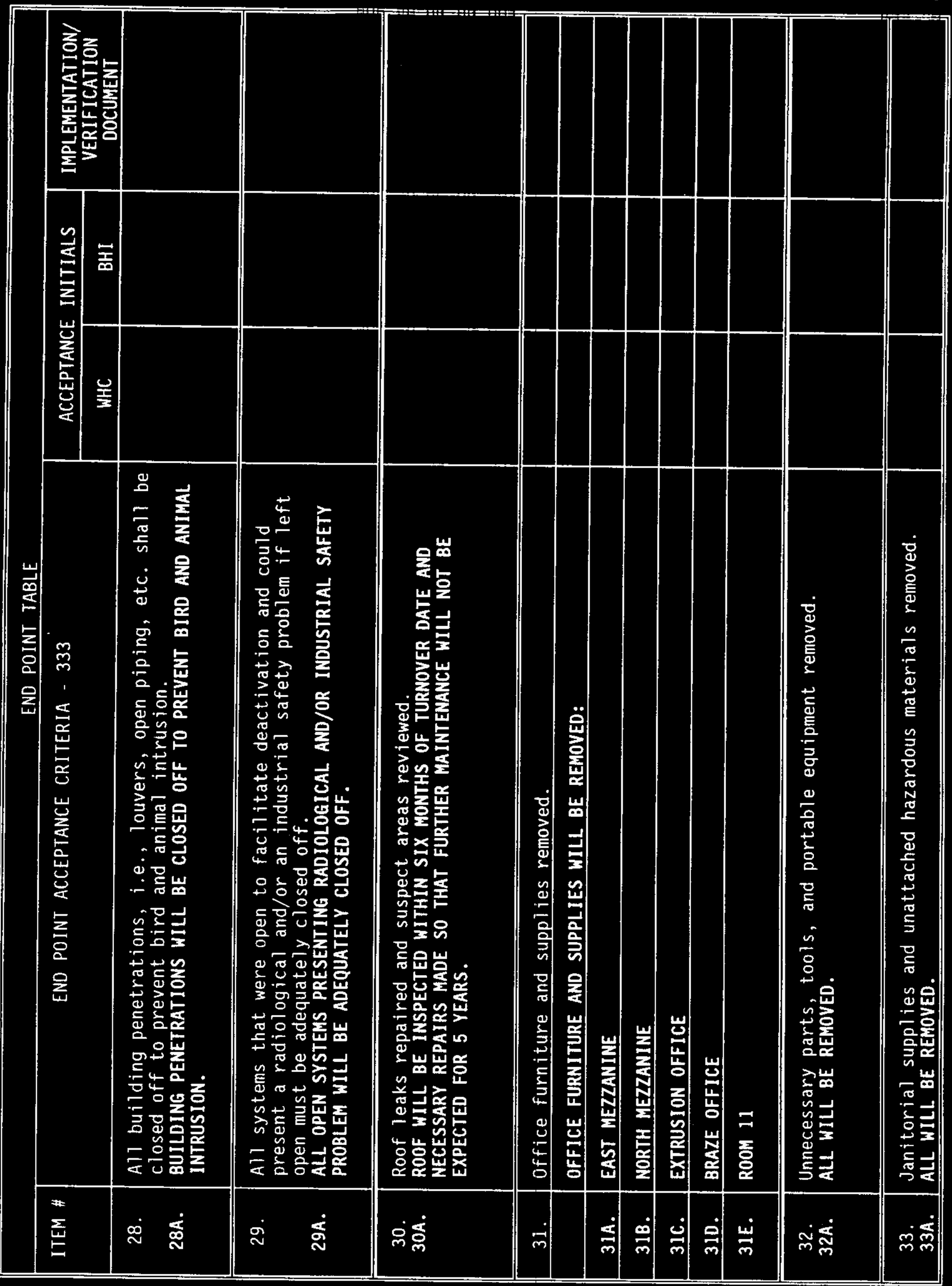


$\therefore \mathrm{V}$.






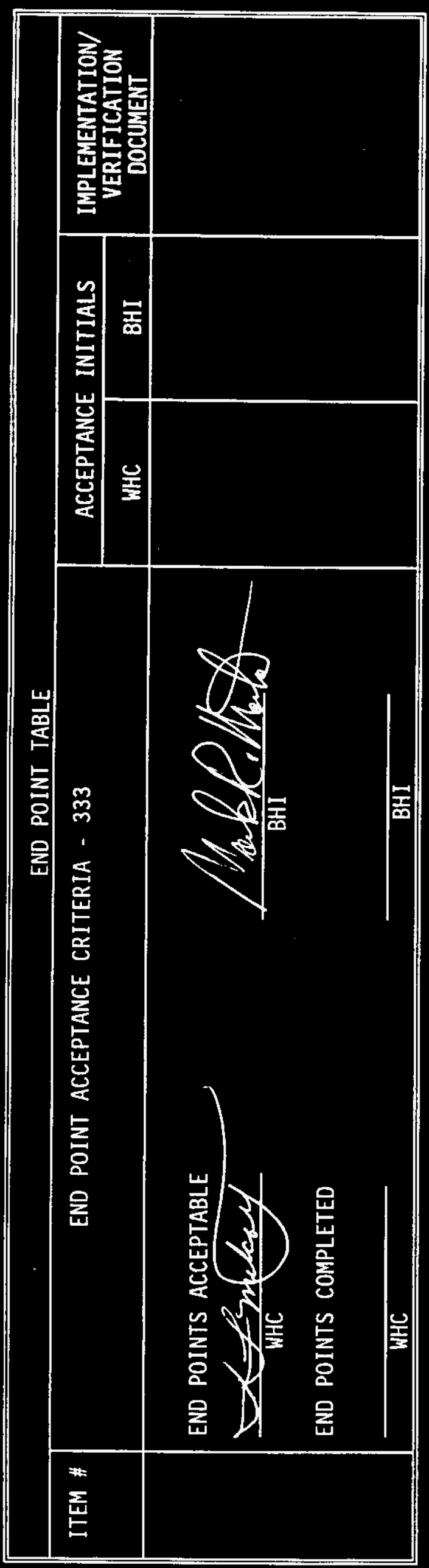

Rev. 0 


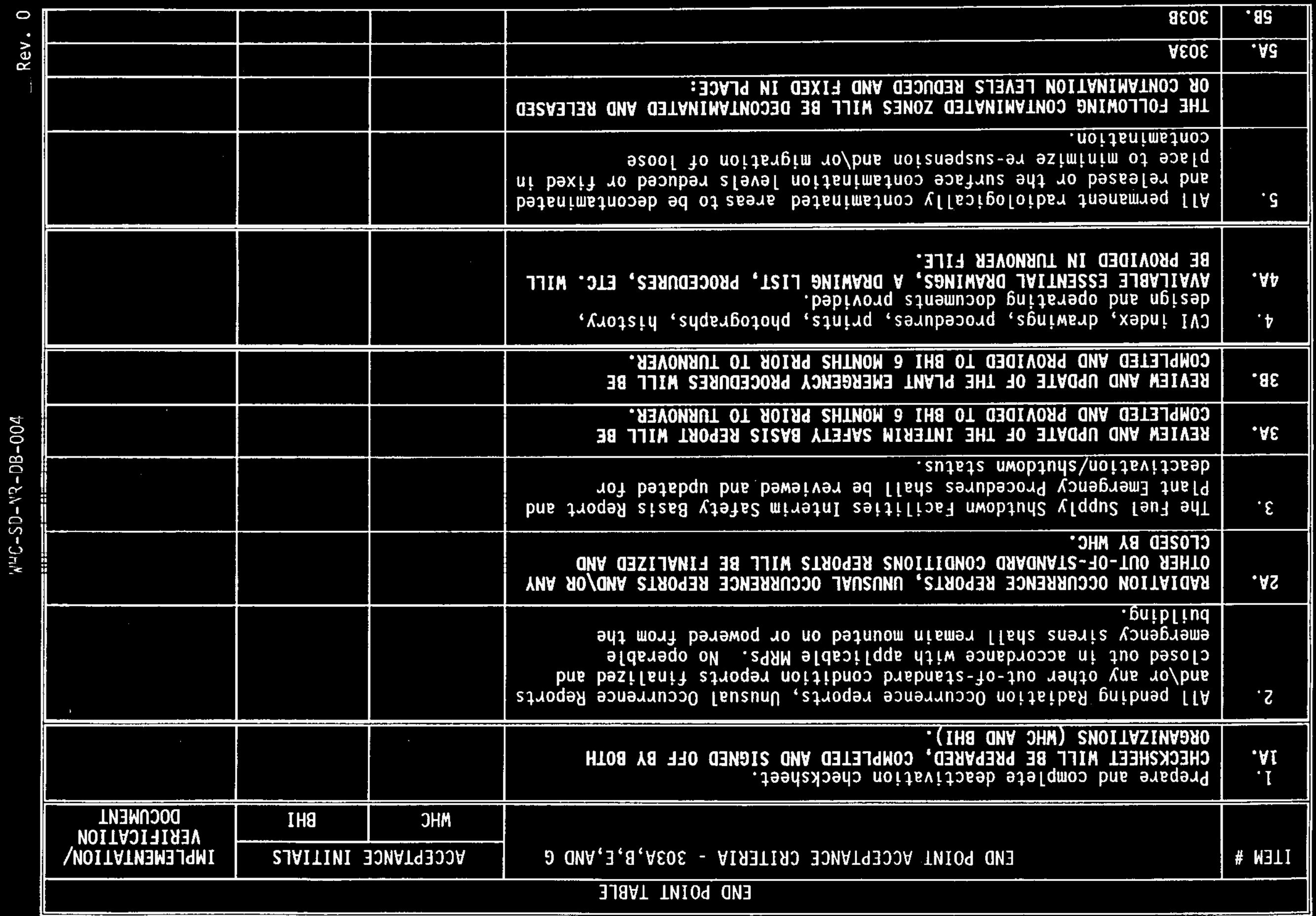




\begin{tabular}{|c|c|c|c|c|}
\hline & & & 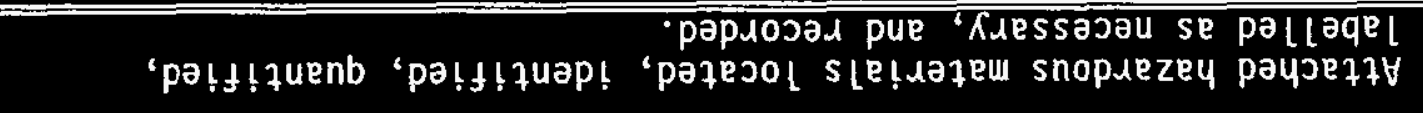 & 'II \\
\hline & & & 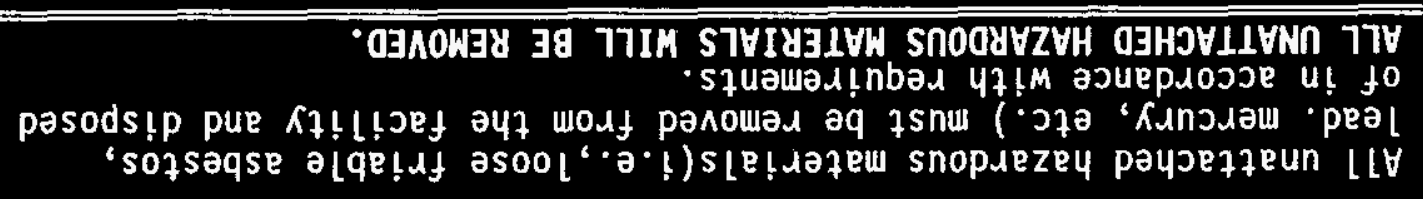 & $\overline{\forall O I}$ \\
\hline & & & 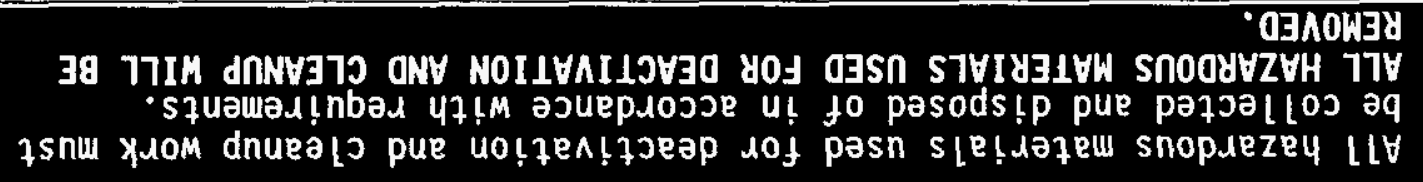 & $\begin{array}{l}\cdot .6 \\
\cdot 6\end{array}$ \\
\hline & & & 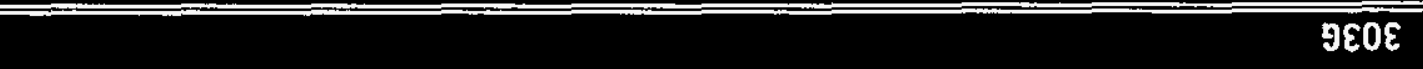 & 08 \\
\hline & & & $3 \varepsilon 0 \varepsilon$ & 58 \\
\hline & & & $8 \varepsilon 0 \varepsilon$ & 88 \\
\hline & & & 'VEOE & $\frac{78}{78}$ \\
\hline & & & 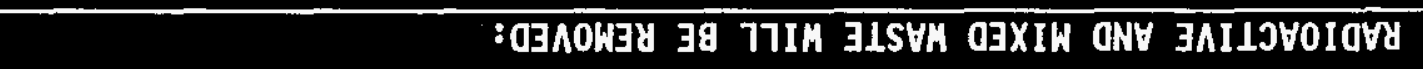 & \\
\hline & & & 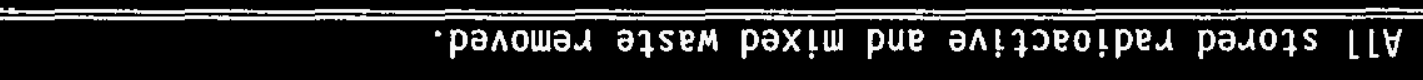 & 8 \\
\hline & & & 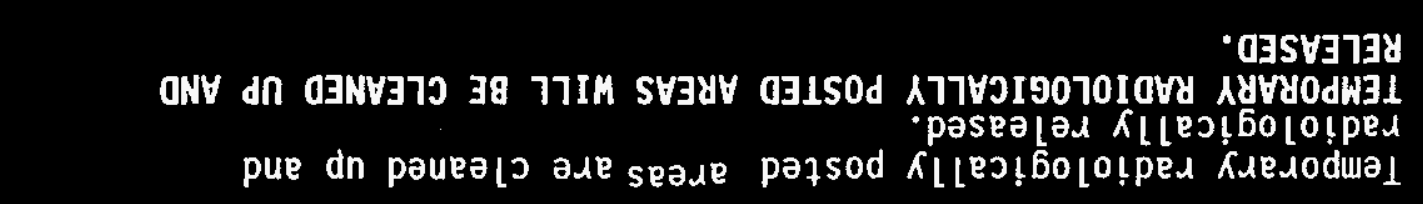 & $\begin{array}{l}\cdot V L \\
\cdot L\end{array}$ \\
\hline & & & 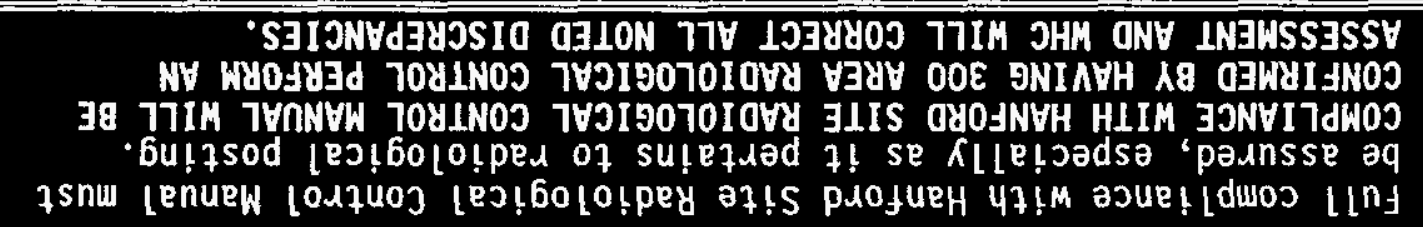 & $\begin{array}{l}\cdot 69 \\
\cdot 9\end{array}$ \\
\hline & & & $\overline{980 \varepsilon}$ & $\overline{09}$ \\
\hline & & & 3EOE & J9 \\
\hline \multirow{2}{*}{ 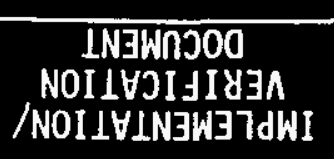 } & IH8 & JHM & \multirow[b]{2}{*}{ 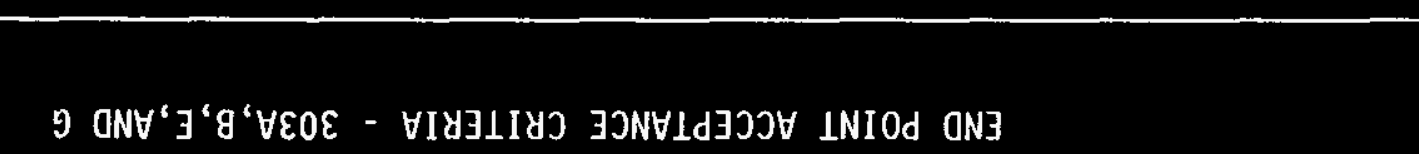 } & \multirow[b]{2}{*}{ \# WBLI } \\
\hline & \multicolumn{2}{|c|}{ STVIIINI JONVIdZJJY } & & \\
\hline
\end{tabular}




\begin{tabular}{|c|c|c|c|c|}
\hline & & $\begin{array}{l}378 \% 917 d d y \\
10 \mathrm{~N}\end{array}$ & 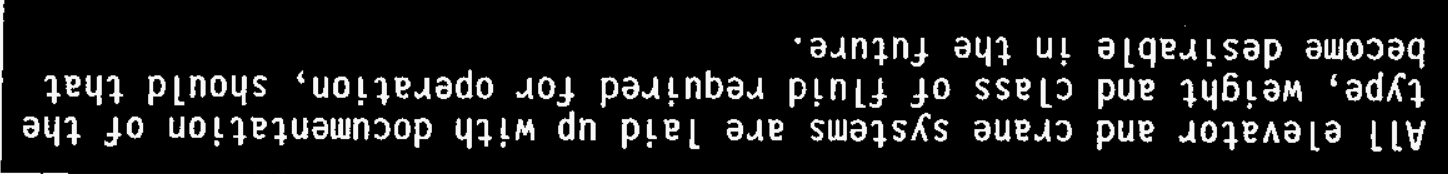 & 'SI \\
\hline & & $\begin{array}{c}379 y 917 d d y \\
10 \mathrm{~W}\end{array}$ & 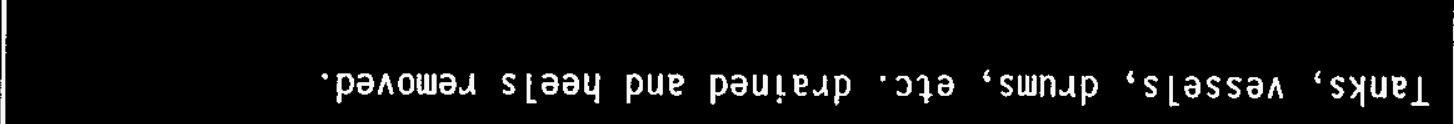 & ' \\
\hline & & & $980 \varepsilon$ & 'AEL \\
\hline & & & $\overline{3 \varepsilon 0 \varepsilon}$ & כEI \\
\hline & & & $8 \varepsilon 0 \varepsilon$ & '8हI \\
\hline & & & Y80E & VEI \\
\hline & & & 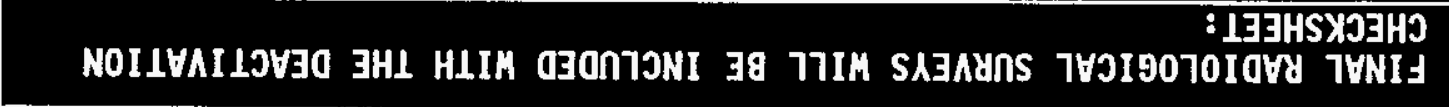 & \\
\hline & & & 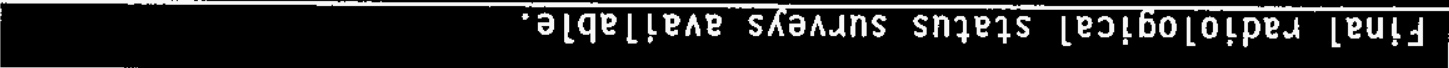 & EI \\
\hline & & & פEQE & az I \\
\hline & & & JE0E & כ2t \\
\hline & & & $8 \varepsilon 0 \varepsilon$ & GQI \\
\hline & & & $\overline{\text { VEOE }}$ & VZI \\
\hline & & & 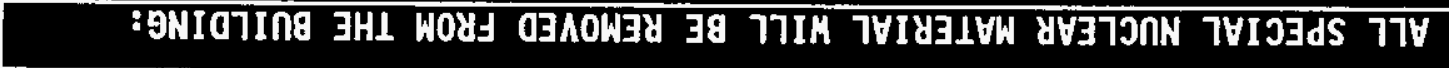 & \\
\hline & & & 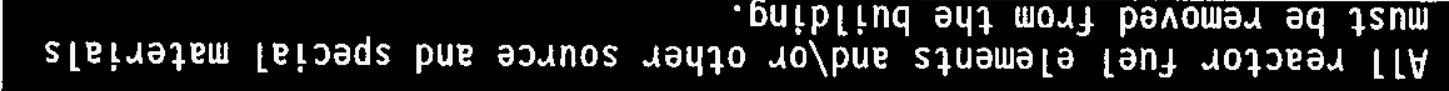 & $\cdot 2 \mathrm{I}$ \\
\hline & & & 980E & att \\
\hline & & & उE0E & गII \\
\hline & & & $8 \varepsilon 0 \varepsilon$ & 'घIt \\
\hline & & & VEOE & 'VII \\
\hline & & & 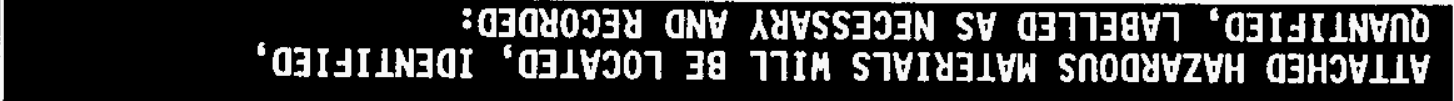 & \\
\hline \multirow{2}{*}{ 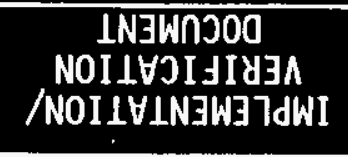 } & IH8 & כHM & \multirow[b]{2}{*}{ 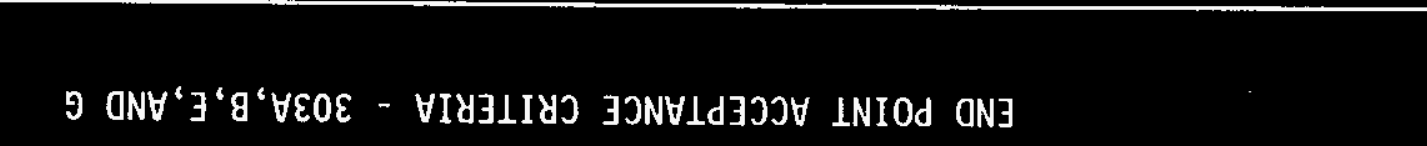 } & \multirow[b]{2}{*}{ \# WELI } \\
\hline & STVILINI & 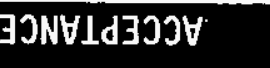 & & \\
\hline
\end{tabular}




\begin{tabular}{|c|c|c|c|c|}
\hline \multicolumn{5}{|c|}{ END POINT TABLE } \\
\hline \multirow[t]{2}{*}{ ITEM \# } & \multirow[t]{2}{*}{ END POINT ACCEPTANCE CRITERIA - 303A, B, E, AND G } & \multicolumn{2}{|c|}{ ACCEPTANCE INITIALS } & \multirow{2}{*}{$\begin{array}{l}\text { IMPLEMENTATION/ } \\
\text { VERIFICATION } \\
\text { DOCUMENT }\end{array}$} \\
\hline & & WHC & $\mathrm{BHI}$ & \\
\hline \multirow[t]{2}{*}{16.} & $\begin{array}{l}\text { Documentation is available confirming that zero energy checks were } \\
\text { made on all de-energized electrical circuits and isolated } \\
\text { pressurized systems e.g., water, service air, steam, etc. using } \\
\text { existing documents to the extent possible. }\end{array}$ & & & \\
\hline & $\begin{array}{l}\text { WATER, STEAM, AND SERVICE AIR WILL BE BLANKED AT LOCATION OUTSIDE } \\
\text { BUILDING: }\end{array}$ & & & \\
\hline $16 A$. & $303 A$ & & & \\
\hline $16 B$. & $303 B$ & & & \\
\hline 16C. & $303 E$ & & & \\
\hline \multirow[t]{2}{*}{160.} & $303 G$ & & & \\
\hline & $\begin{array}{l}\text { IF ELECTRICAL SERVICE IS NOT DISCONNECTED BY CUTTING THE FEED } \\
\text { CABLES, ENERGY STATUS WILL BE DOCUMENTED ON ENERGIZED ELECTRICAL } \\
\text { SYSTEMS: }\end{array}$ & & & \\
\hline $16 E$. & $303 A$ & & & \\
\hline $16 F$. & 303B & & & \\
\hline 16G. & $303 E$ & & & \\
\hline $16 \mathrm{H}$. & 3036 & & & \\
\hline $17 \mathrm{~A}$. & $\begin{array}{l}\text { Electrical systems reduced to that necessary for S\&M and subsequent } \\
\text { D\&D. This includes removal of emergency light fixtures and the } \\
\text { associated batteries. Where appropriate, centralize the remaining } \\
\text { electrical services to a single point. } \\
\text { ELECTRICAL SYSTENS WILL BE APPROPRIATELY ISOLATED AND THE ISOLATION } \\
\text { DOCUMENTED, INCLUDING ISOLATION LOCATION(S). }\end{array}$ & & & \\
\hline 18. & $\begin{array}{l}\text { All heating, ventilation and air conditioning supply and exhaust air } \\
\text { systems shut down and de-energized. This criteria includes } \\
\text { evaluation and, as appropriate, the shutdown of the High-efficiency } \\
\text { particulate air-filtered (HEPA) ventilation exhaust from process } \\
\text { areas. }\end{array}$ & $\begin{array}{l}\text { NOT } \\
\text { APPLICABLE }\end{array}$ & & \\
\hline
\end{tabular}




\begin{tabular}{|c|c|c|c|c|}
\hline \multicolumn{5}{|c|}{ END POINT TABLE } \\
\hline \multirow[t]{2}{*}{ ITEM \# } & \multirow[t]{2}{*}{ END POINT ACCEPTANCE CRITERIA - 303A, B, E, AND G } & \multicolumn{2}{|c|}{ ACCEPTANCE INITIALS } & \multirow{2}{*}{$\begin{array}{l}\text { IMPLEMENTATION } \\
\text { VERIFICATION } \\
\text { DOCUMENT }\end{array}$} \\
\hline & & WHC & BHI & \\
\hline 19. & All unneeded equipment shut down. & $\begin{array}{c}\text { NOT } \\
\text { APPLICABLE } \\
\end{array}$ & & \\
\hline 20. & Fire protection systems downgraded. & & & \\
\hline 20A. & ALL PORTABLE FIRE EXTINGUISHERS WILL BE REMOVED FRON THE BUILDING. & & & \\
\hline 20B. & $\begin{array}{l}\text { THE FIRE SPRINKLER SYSTEM WILL BE DRAINED AND THE FIRE PROTECTION } \\
\text { RISER WILL BE BLANKED AT THE PIV. }\end{array}$ & & & \\
\hline $20 \mathrm{C}$. & THE FIRE ALARM PANEL AND ALARM SYSTEM WILL BE DISCONNECTED. & & & \\
\hline 21. & $\begin{array}{l}\text { The radiation space monitoring system should be reduced to a level } \\
\text { required by the Health Physics organization. }\end{array}$ & $\begin{array}{l}\text { NOT } \\
\text { APPLICABLE }\end{array}$ & & \\
\hline 22. & $\begin{array}{l}\text { The continuous air monitoring system should be reduced to a level } \\
\text { required by the Health Physics organization. }\end{array}$ & $\begin{array}{l}\text { NOT } \\
\text { APPLICABLE }\end{array}$ & & \\
\hline 23. & $\begin{array}{l}\text { The criticality monitoring and alarm system should be deactivated. } \\
\text { THE CRITICALITY MONITORING AND ALARM SYSTEM WILL BE DEACTIVATED. }\end{array}$ & & & \\
\hline 24. & $\begin{array}{l}\text { Reduce or eliminate the electrical, service air, and water supply } \\
\text { services to the building in accordance with the requirements for } \\
\text { S\&M. }\end{array}$ & & & \\
\hline & WATER SUPPLY WILL BE BLANKED AT LOCATION OUTSIDE THE BUILDING: & & & \\
\hline 24A. & $303 A$ & & & \\
\hline 24B. & $303 B$ & & & \\
\hline 24C. & $303 E$ & & & \\
\hline 240. & $303 G$ & & & \\
\hline $24 E$. & ELECTRICAL SERVICE WILL BE REDUCED PER ITEN \#17. & & & \\
\hline 25. & Remove all emergency 1 ighting and dispose of associated batteries. & & & \\
\hline
\end{tabular}




\begin{tabular}{|c|c|c|c|c|}
\hline \multicolumn{5}{|c|}{ END POINT TABLE } \\
\hline \multirow[t]{3}{*}{ ITEM \# } & \multirow[t]{2}{*}{ END POINT ACCEPTANCE CRITERIA - 303A, B, E, AND G } & \multicolumn{2}{|c|}{ ACCEPTANCE INITIALS } & \multirow{2}{*}{$\begin{array}{c}\text { IMPLEMENTATION } \\
\text { VERIFICATION } \\
\text { DOCUMENT }\end{array}$} \\
\hline & & WHC & BHI & \\
\hline & $\begin{array}{l}\text { ALL ENERGENCY LIGHTING WILL BE REMOVED AND ASSOCIATED BATTERIES } \\
\text { DISPOSED: }\end{array}$ & & & \\
\hline 25A. & $303 A$ & & & \\
\hline $25 B$. & $303 B$ & & & \\
\hline $25 \mathrm{C}$. & $303 E$ & & & \\
\hline 250. & 3036 & & & \\
\hline \multirow[t]{2}{*}{26.} & The building steam system should be deactivated and tagged. & & & \\
\hline & STEAM BLANKED AT BUILDING MAIN CONNECTION OUTSIDE THE BUILDING: & & & \\
\hline 26A. & 303A & & & \\
\hline 268. & 303B & $\begin{array}{c}\text { NOT } \\
\text { APPLICABLE }\end{array}$ & & \\
\hline $26 C$. & 303E & $\begin{array}{l}\text { NOT } \\
\text { APPLICABLE }\end{array}$ & & \\
\hline 260. & 3036 & $\begin{array}{l}\text { MOT } \\
\text { APPLICABLE }\end{array}$ & & \\
\hline 27. & $\begin{array}{l}\text { All effluent flow routes to disposal sites are isolated by sealing } \\
\text { at the building. }\end{array}$ & $\begin{array}{l}\text { NOT } \\
\text { APPLICABLE }\end{array}$ & & \\
\hline \multirow[t]{2}{*}{28.} & $\begin{array}{l}\text { All building penetrations, i.e., louvers, open piping, etc. shall be } \\
\text { closed off to prevent bird and animal intrusion. }\end{array}$ & & & \\
\hline & $\begin{array}{l}\text { BUILDING PENETRATIONS WILL BE CLOSED OFF TO PREVENT BIRD AND ANIMAL } \\
\text { INTRUSION: }\end{array}$ & & & \\
\hline 28A. & $303 A$ & & & \\
\hline 288. & $303 B$ & & & \\
\hline $28 \mathrm{C}$. & $303 E$ & & & \\
\hline 280. & 3039 & & & \\
\hline
\end{tabular}




\begin{tabular}{|c|c|c|c|c|}
\hline \multicolumn{5}{|c|}{ END POINT TABLE } \\
\hline \multirow{2}{*}{ ITEM \# } & \multirow{2}{*}{ END POINT ACCEPTANCE CRITERIA - 303A, B, E, AND G } & \multicolumn{2}{|c|}{ ACCEPTANCE INITIALS } & \multirow{2}{*}{$\begin{array}{l}\text { IMPLEMENTATION/ } \\
\text { VERIFICATION } \\
\text { DOCUMENT } \\
\end{array}$} \\
\hline & & WHC & BHI & \\
\hline 29. & $\begin{array}{l}\text { Al1 systems that were open to facilitate deactivation and could } \\
\text { present a radiological and/or an industrial safety problem if left } \\
\text { open must be adequately closed off. } \\
\text { ALL OPEN SYSTEMS PRESENTING RADIOLOGICAL AND/OR INDUSTRIAL SAFETY } \\
\text { PROBLEM WILL BE ADEQUATELY CLOSED OFF. }\end{array}$ & & & \\
\hline \multirow[t]{2}{*}{30.} & Roof leaks repaired and suspect areas reviewed. & & & \\
\hline & $\begin{array}{l}\text { ROOF WILL BE INSPECTED WITHIN SIX MONTHS OF TURNOVER DATE AND } \\
\text { NECESSARY REPAIRS MADE SO THAT FURTHER MAINTENANCE WILL NOT BE } \\
\text { EXPECTED FOR } 5 \text { YEARS. }\end{array}$ & & & \\
\hline 30A. & $303 A$ & & & \\
\hline $30 \mathrm{~B}$. & $303 B$ & & & \\
\hline $30 \mathrm{C}$. & $303 E$ & & & \\
\hline 300. & 3036 & & & \\
\hline 31. & Office furniture and supplies removed. & $\begin{array}{c}\text { NOT } \\
\text { APPLICABLE }\end{array}$ & & \\
\hline 32. & Unnecessary parts, tools, and portable equipment removed. & $\begin{array}{l}\text { NOT } \\
\text { APPLICABLE }\end{array}$ & & \\
\hline \multirow[t]{2}{*}{33.} & Janitorial supplies and unattached hazardous materials removed. & & & \\
\hline & ALL WILL BE RENOVED: & & & \\
\hline 33A. & $303 A$ & & & \\
\hline $33 B$. & $303 B$ & & & \\
\hline 33C. & $303 E$ & & & \\
\hline 330. & 3036 & & & \\
\hline
\end{tabular}




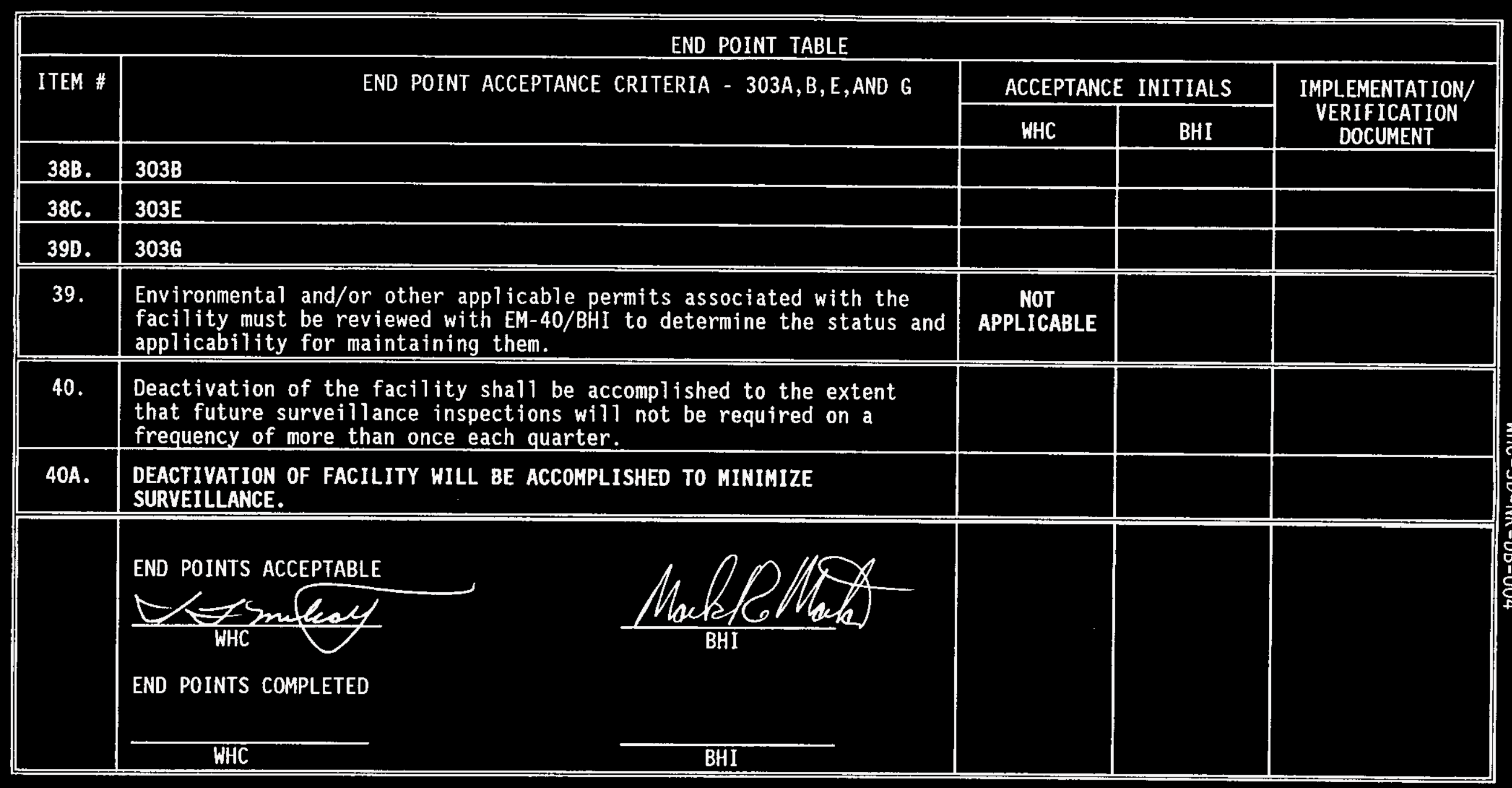


END POINT TABLE

\begin{tabular}{|c|c|c|c|c|}
\hline \multirow[t]{2}{*}{ ITEM \# } & \multirow[t]{2}{*}{ END POINT ACCEPTANCE CRITERIA - 3716} & \multicolumn{2}{|c|}{ ACCEPTANCE INITIALS } & \multirow{2}{*}{$\begin{array}{l}\text { IMPLEMENTATION/ } \\
\text { VERIFICATION } \\
\text { DOCUMENT }\end{array}$} \\
\hline & & WHC & BHI & \\
\hline 1. & $\begin{array}{l}\text { Prepare and complete deactivation checksheet, } \\
\text { CHECKSHEET WILL BE PREPARED, COMPLETED AND SIGNED OFF BY BOTH } \\
\text { ORGANIZATIONS (WHC AND BHI). }\end{array}$ & & & . \\
\hline 2. & $\begin{array}{l}\text { Al1 pending Radiation Occurrence reports, Unusual Occurrence Reports } \\
\text { and } \text { or any other out-of-standard condition reports finalized and } \\
\text { closed out in accordance with applicable MRPs. No operable } \\
\text { emergency sirens shall remain mounted on or powered from the } \\
\text { building. }\end{array}$ & & & \\
\hline $2 A$ & $\begin{array}{l}\text { RADIATION OCCURRENCE REPORTS, UNUSUAL OCCURRENCE REPORTS AND OR ANY } \\
\text { OTHER OUT-OF-STANDARD CONDITIONS REPORTS WILL BE FINALIZED AND } \\
\text { CLOSED BY WHC. }\end{array}$ & & & \\
\hline 3. & $\begin{array}{l}\text { The Fuel Supply Shutdown Facilities Interim Safety Basis Report and } \\
\text { Plant Emergency Procedures shall be reviewed and updated for } \\
\text { deactivation/shutdown status. }\end{array}$ & & & \\
\hline $3 A$. & $\begin{array}{l}\text { REVIEH AND UPDATE OF THE INTERIM SAFETY BASIS REPORT WILL BE } \\
\text { CONPLETED AND PROVIDED TO BHI } 6 \text { MONTHS PRIOR TO TURNOVER. }\end{array}$ & & & \\
\hline 3B. & $\begin{array}{l}\text { REVIEH AND UPDATE OF THE PLANT EMERGENCY PROCEDURES HILL BE } \\
\text { COHPLETED AND PROVIDED TO BHI } 6 \text { MONTHS PRIOR TO TURNOVER. }\end{array}$ & & & \\
\hline 4. & $\begin{array}{l}\text { CVI index, drawings, procedures, prints, photographs, history, } \\
\text { design and operat ing documents provided. } \\
\text { AVAILABLE ESSENTIAL DRAWINGS, A DRAWING LIST, PROCEDURES, ETC. HILL } \\
\text { BE PROVIDED IN TURNOVER FILE. }\end{array}$ & & & \\
\hline 5. & $\begin{array}{l}\text { A11 permanent radiologically contaminated areas to be decontaminated } \\
\text { and released or the surface contamination levels reduced or fixed in } \\
\text { place to minimize re-suspension and } \backslash \text { or migration of loose } \\
\text { contamination. } \\
\text { ANY CONTAMINATED ZONES WILL BE DECONTANINATED AND RELEASED OR } \\
\text { CONTANINATION LEVELS REDUCED AND FIXED IN PLACE. }\end{array}$ & & & \\
\hline
\end{tabular}




\begin{tabular}{|c|c|c|c|c|}
\hline \multicolumn{5}{|c|}{ END POINT TABLE } \\
\hline \multirow[t]{2}{*}{ ITEM \# } & \multirow[t]{2}{*}{ END POINT ACCEPTANCE CRITERIA - 3716} & \multicolumn{2}{|c|}{ ACCEPTANCE INITIALS } & \multirow{2}{*}{$\begin{array}{l}\text { IMPLEMENTATION/ } \\
\text { VERIFICATION } \\
\text { DOCUMENT }\end{array}$} \\
\hline & & WHC & BHI & \\
\hline 6.: & $\begin{array}{l}\text { Ful1 compliance with Hanford Site Radiological Control Manual must } \\
\text { be assured, especially as it pertains to radiological post ing. } \\
\text { COMPLIANCE WITH HANFORD SITE RADIOLOGICAL CONTROL MANUAL WILL BE } \\
\text { CONFIRMED BY HAVING } 300 \text { AREA RADIOLOGICAL CONTROL PERFORM AN } \\
\text { ASSESSWENT AND WHC WILL CORRECT ALL NOTED DISCREPANCIES. }\end{array}$ & & & \\
\hline 7. & $\begin{array}{l}\text { Temporary radiologically posted areas are cleaned up and } \\
\text { radiologically released. } \\
\text { TENPORARY RADIOLOGICALLY POSTED AREAS WILL BE CLEANED UP AND } \\
\text { RELEASED. }\end{array}$ & & & \\
\hline 8. & A11 stored radioactive and mixed waste removed. & $\begin{array}{c}\text { NOT } \\
\text { APPLICABLE } \\
\end{array}$ & & \\
\hline $\begin{array}{l}9 . \\
9 A .\end{array}$ & $\begin{array}{l}\text { A1l hazardous materials used for deactivation and cleanup work must } \\
\text { be collected and disposed of in accordance with requirements. } \\
\text { ALL. HAZARDOUS MATERIALS USED FOR DEACTIVATION AND CLEANUP WIL BE } \\
\text { REMOVED. }\end{array}$ & & & \\
\hline 10. & $\begin{array}{l}\text { A11 unattached hazardous materials (i.e., loose friable asbestos, } \\
\text { lead. mercury, etc.) must be removed from the facility and disposed } \\
\text { of in accordance with requirements. } \\
\text { ALL UNATTACHED HAZARDOUS MATERIALS WILL BE REMOVED. }\end{array}$ & & & \\
\hline 11. & $\begin{array}{l}\text { Attached hazardous materials located, ident ified, quantified, } \\
\text { labelled as necessary, and recorded. } \\
\text { ATTACHED HAZARDOUS MATERIALS WILL BE LOCATED, IDENTIFIED, } \\
\text { QUANTIFIED, LABELLED AS NECESSARY AND RECORDED. }\end{array}$ & & & \\
\hline $\begin{array}{l}12 . \\
12 \mathrm{~A} .\end{array}$ & $\begin{array}{l}\text { A11 reactor fuel elements and \or other source and special materials } \\
\text { must be removed from the building. } \\
\text { ALL SPECIAL NUCLEAR MATERIAL WILL BE REMOVED FROM THE BUILDING. }\end{array}$ & & & \\
\hline $\begin{array}{l}13 . \\
13 A\end{array}$ & $\begin{array}{l}\text { Final radiological status surveys available. } \\
\text { FINAL RADIOLOGICAL SURVEYS WILL BE INCLUDED WITH THE DEACTIVATION } \\
\text { CHECKSHEET. }\end{array}$ & & & \\
\hline
\end{tabular}




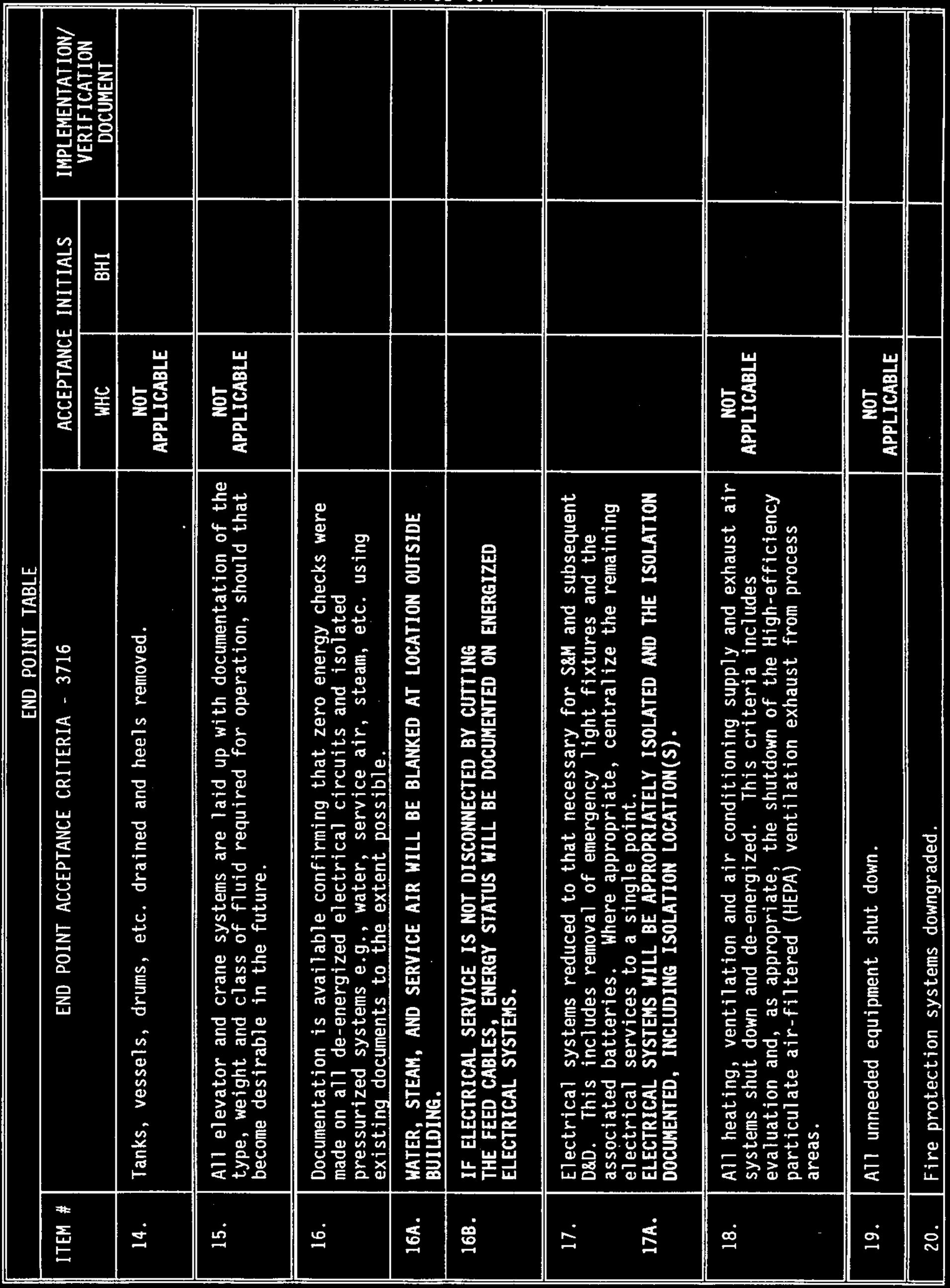




\begin{tabular}{|c|c|c|c|c|}
\hline \multicolumn{5}{|c|}{ END POINT TABLE } \\
\hline \multirow[t]{2}{*}{ ITEM \# } & \multirow[t]{2}{*}{ END POINT ACCEPTANCE CRITERIA - 3716} & \multicolumn{2}{|c|}{ ACCEPTANCE INITIALS } & \multirow{2}{*}{$\begin{array}{l}\text { IMPLEMENTATION/ } \\
\text { VERIFICATION } \\
\text { DOCUMENT }\end{array}$} \\
\hline & & WHC & BHI & \\
\hline 20A. & ALL PORTABLE FIRE EXTINGUISHERS UILL BE REMOVED FROM THE BUILDING. & & & \\
\hline $20 \mathrm{~B}$. & $\begin{array}{l}\text { THE FIRE SPRINKLER SYSTEM WILL BE DRAINED AND THE FIRE PROTECTION } \\
\text { RISER WILL BE BLANKED AT THE PIV. }\end{array}$ & & & \\
\hline 21. & $\begin{array}{l}\text { The radiation space monitoring system should be reduced to a level } \\
\text { required by the Health Physics organization. }\end{array}$ & $\begin{array}{l}\text { NOT } \\
\text { APPLICABLE }\end{array}$ & & \\
\hline 22. & $\begin{array}{l}\text { The continuous air monitoring system should be reduced to a level } \\
\text { required by the Health Physics organization. }\end{array}$ & $\begin{array}{l}\text { NOT } \\
\text { APPLICABLE }\end{array}$ & & \\
\hline $\begin{array}{l}23 . \\
23 A .\end{array}$ & $\begin{array}{l}\text { The criticality monitoring and alarm system should be deactivated. } \\
\text { THE CRITICALITY MONITORING AND ALARM SYSTEM WILL BE DEACTIVATED. }\end{array}$ & & & \\
\hline 24. & $\begin{array}{l}\text { Reduce or eliminate the electrical, service air, and water supply } \\
\text { services to the building in accordance with the requirements for } \\
\text { S\&M. }\end{array}$ & & & \\
\hline 24A. & WATER SUPPLY WILL BE BLANKED AT LOCATION OUTSIDE THE BUILDING. & & & \\
\hline 24B. & ELECTRICAL SERVICE WILL BE REDUCED PER ITEM \#17. & & & \\
\hline 24C. & SERVICE AIR WILL BE BLANKED AT ENTRANCE TO THE BUILOING. & & & \\
\hline 25. & $\begin{array}{l}\text { Remove all emergency } 1 \text { ighting and dispose of associated batteries. } \\
\text { ALL. EMERGENCY LIGHTING WILL BE REMOVEO AND ASSOCIATED BATTERIES } \\
\text { DISPOSED. }\end{array}$ & & & \\
\hline $\begin{array}{l}26 . \\
26 A .\end{array}$ & $\begin{array}{l}\text { The building steam system should be deactivated and tagged. } \\
\text { STEAN BLANKED AT BUILDING MAIN CONNECTION OUTSIDE THE BUILDING. }\end{array}$ & & & \\
\hline 27. & $\begin{array}{l}\text { All effluent flow routes to disposal sites are isolated by sealing } \\
\text { at the building. }\end{array}$ & & & \\
\hline 27A. & $\begin{array}{l}\text { EFFLUENT FLON ROUTES TO THE PROCESS SEWER WILL BE PLUGGED AT THE } \\
\text { BUILDING. }\end{array}$ & & & \\
\hline
\end{tabular}




\begin{tabular}{|c|c|c|c|c|}
\hline \multicolumn{5}{|c|}{ END POINT TABLE } \\
\hline \multirow[t]{2}{*}{ ITEM \# } & \multirow[t]{2}{*}{ END POINT ACCEPTANCE CRITERIA - 3716} & \multicolumn{2}{|c|}{ ACCEPTANCE INITIALS } & \multirow{2}{*}{$\begin{array}{l}\text { IMPLEMENTAT ION/ } \\
\text { VERIF ICATION } \\
\text { DOCUMENT }\end{array}$} \\
\hline & & $\mathrm{HHC}$ & BHI & \\
\hline 27B. & EFFLUENT FLOW ROUTES TO THE SANITARY SEWER WILL BE PLUGGED. & & & \\
\hline $\begin{array}{l}28 . \\
28 A\end{array}$ & $\begin{array}{l}\text { All building penetrations, i.e., louvers, open piping, etc. shall be } \\
\text { closed off to prevent bird and animal intrusion. } \\
\text { BUILDING PENETRATIONS WILL BE CLOSED OFF TO PREVENT BIRD AND ANIMAL } \\
\text { INTRUSION. }\end{array}$ & & & \\
\hline 29. & $\begin{array}{l}\text { A11 systems that were open to facilitate deactivation and could } \\
\text { present a radiological and/or an industrial safety problem if left } \\
\text { open must be adequately closed off. } \\
\text { ALL OPEN SYSTEMS PRESENTING RADIOLOGICAL AND/OR INDUSTRIAL SAFETY } \\
\text { PROBLEM WILL. BE ADEQUATELY CLOSED OFF. }\end{array}$ & & & \\
\hline $\begin{array}{l}30 . \\
30 A .\end{array}$ & $\begin{array}{l}\text { Roof leaks repaired and suspect areas reviewed. } \\
\text { ROOF WILL BE INSPECTED WITHIN SIX MONTHS OF TURNOVER DATE AND } \\
\text { MECESSARY REPAIRS MADE SO THAT FURTHER MAINTENANCE WILL NOT BE } \\
\text { EXPECTED FOR } 5 \text { YEARS. }\end{array}$ & & & \\
\hline 31. & Office furniture and supplies removed. & $\begin{array}{c}\text { NOT } \\
\text { APPLICABLE }\end{array}$ & & \\
\hline 32. & Unnecessary parts, tools, and portable equipment removed. & $\begin{array}{c}\text { NOT } \\
\text { APPLICABLE }\end{array}$ & & \\
\hline 33. & Janitorial supplies and unattached hazardous materials removed. & $\begin{array}{c}\text { NOT } \\
\text { APPLICABLE }\end{array}$ & & \\
\hline $\begin{array}{l}34 . \\
34 A .\end{array}$ & $\begin{array}{l}\text { Loose and } \text { or damaged asbestos removed or stabilized. } \\
\text { LOOSE AND OR DAMAGED ASBESTOS WILL BE REMOVED OR STABILIZED. } \\
\text { ASBESTOS CONDITION ASSESSMENT WILL BE UPDATED WITHIN THREE HONTHS OF } \\
\text { TURNOVER. }\end{array}$ & & & \\
\hline
\end{tabular}




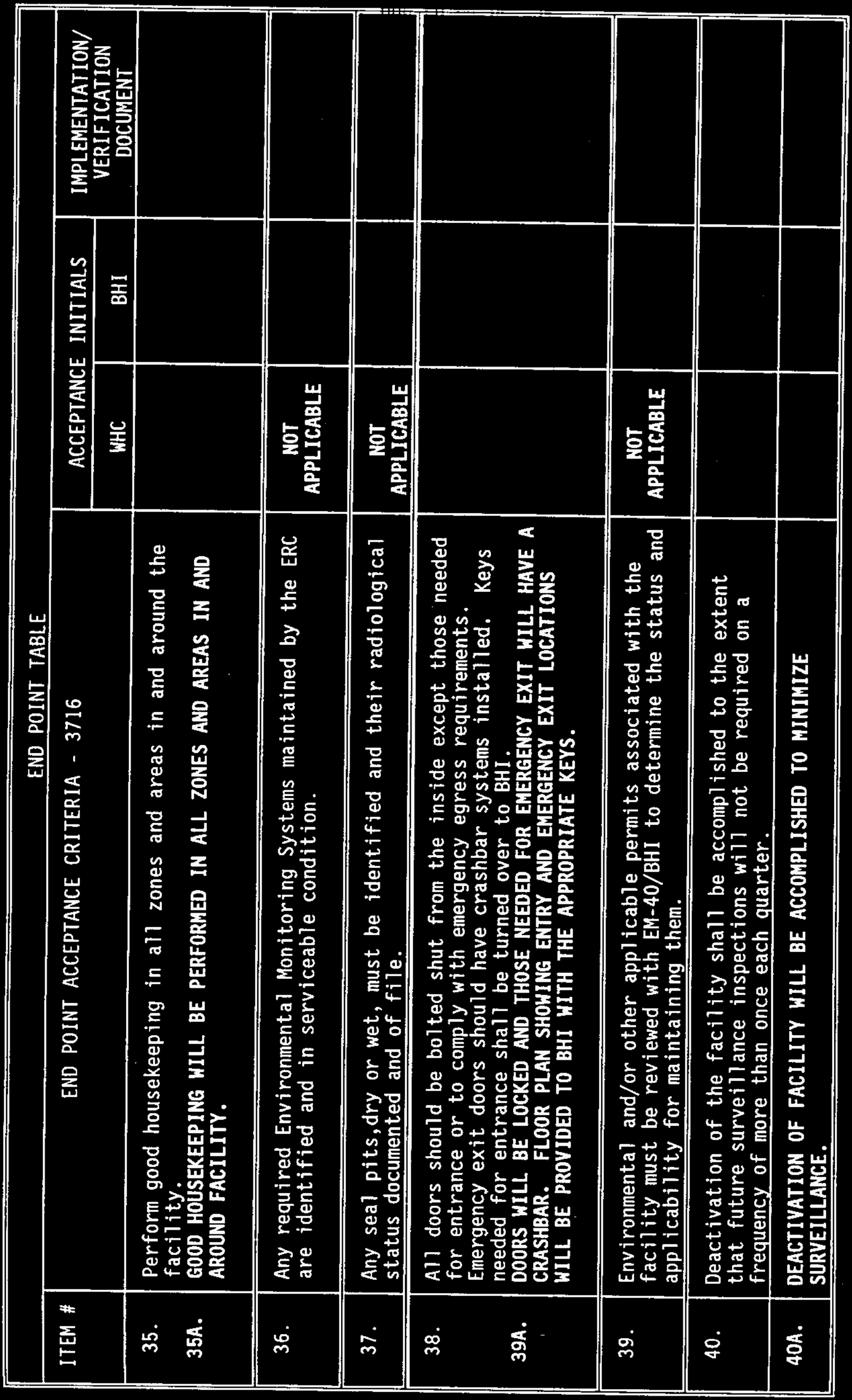




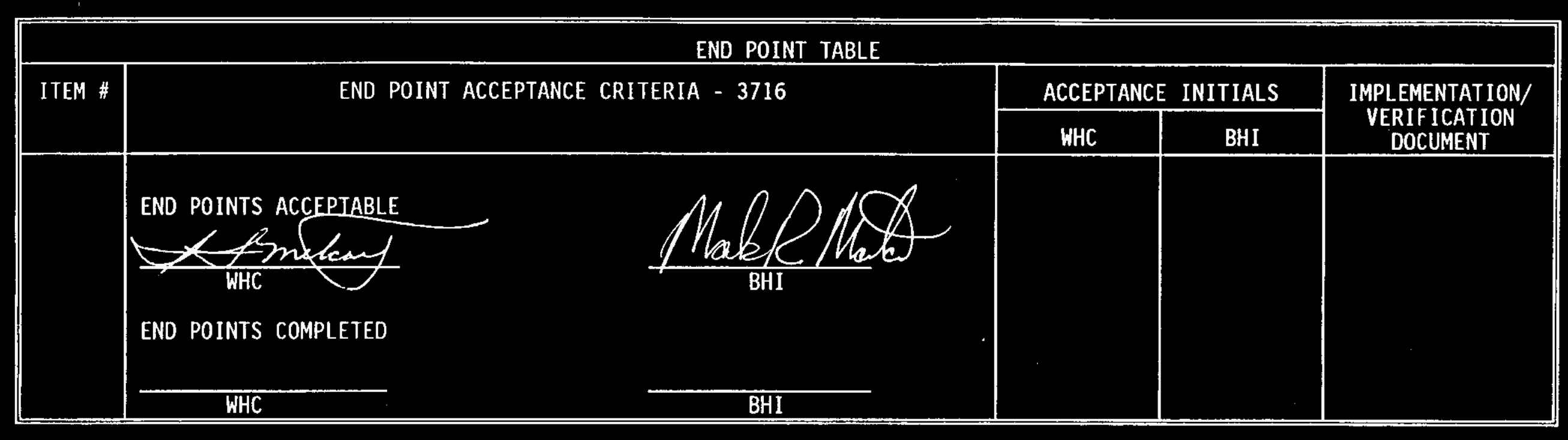




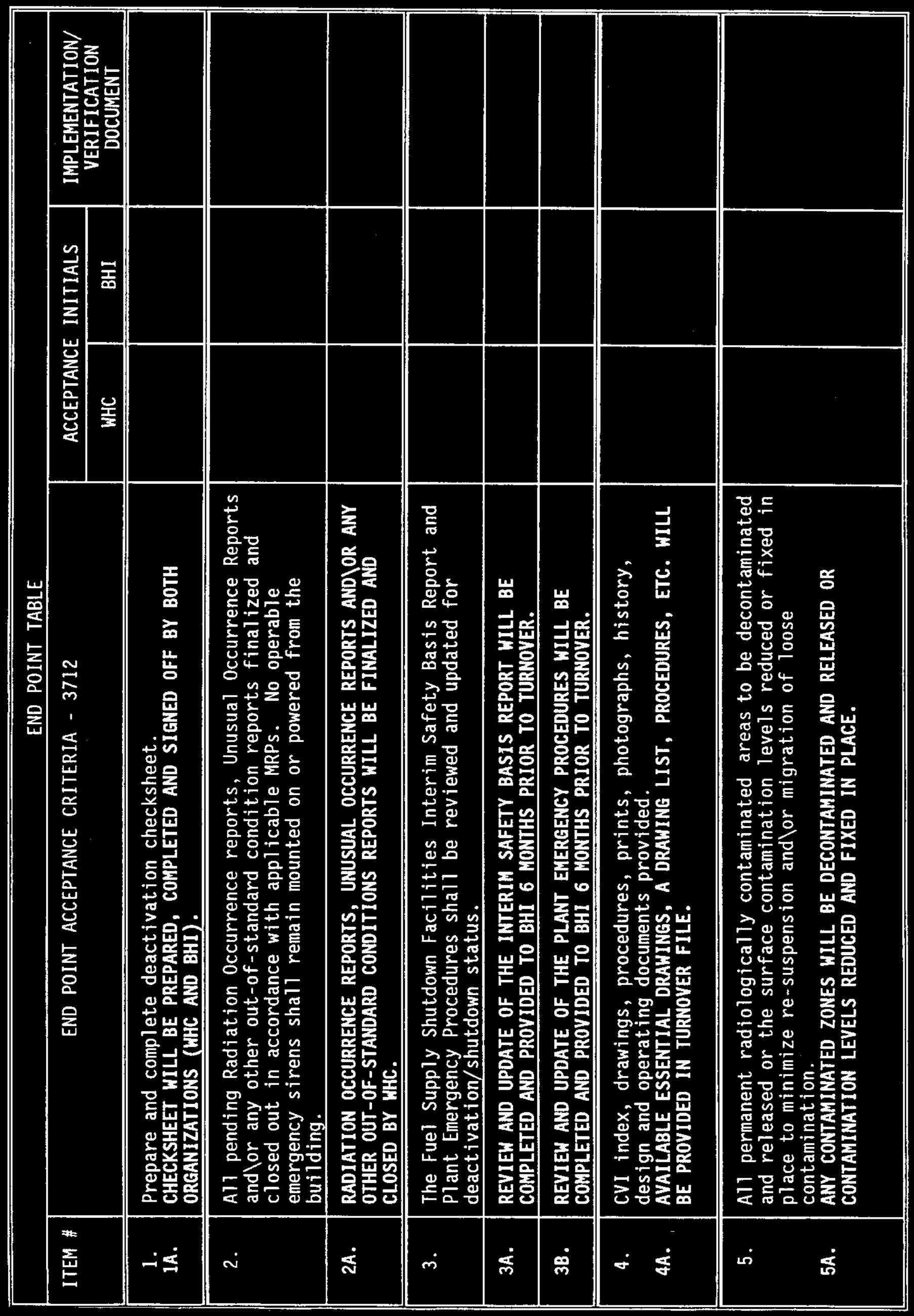




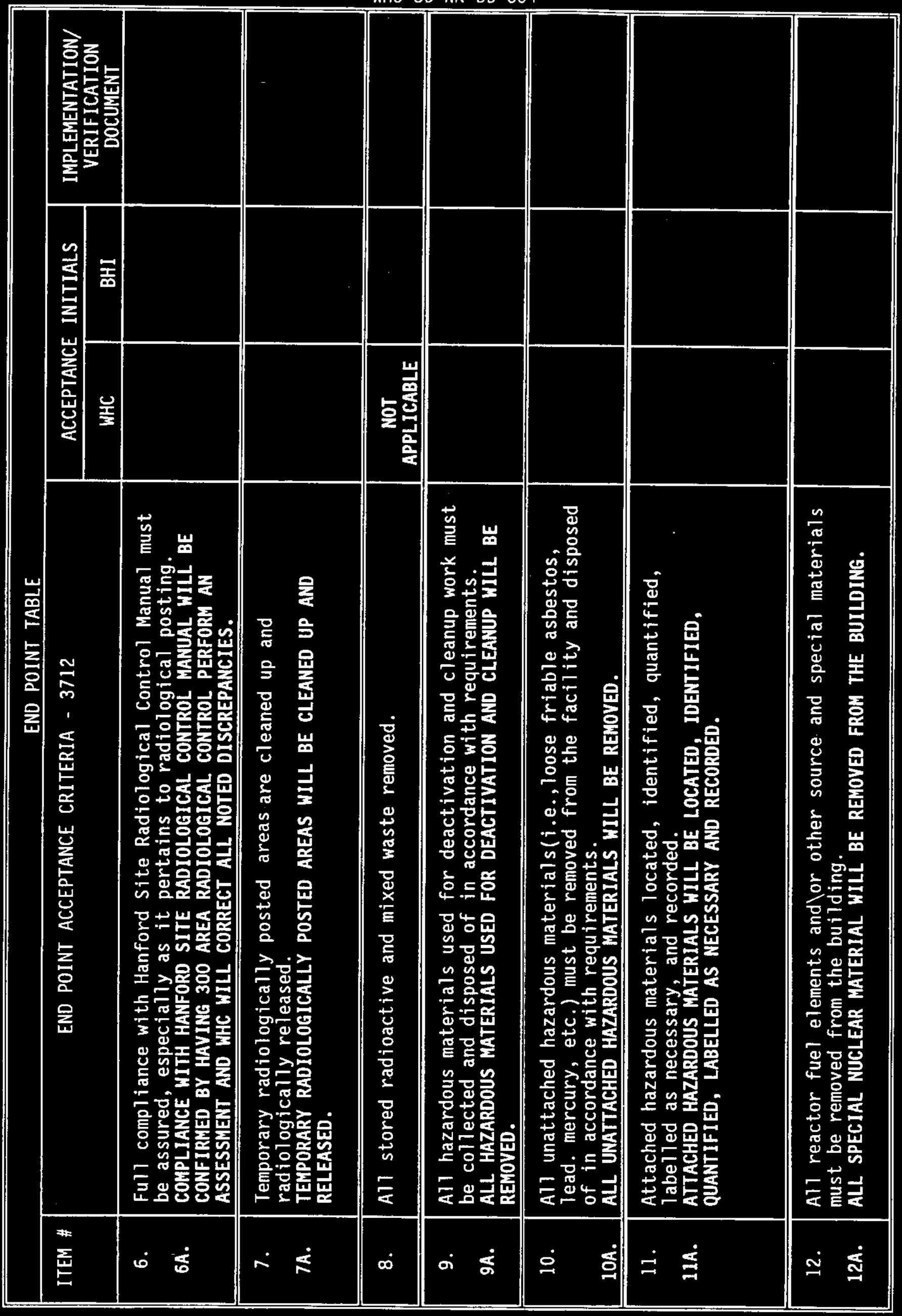




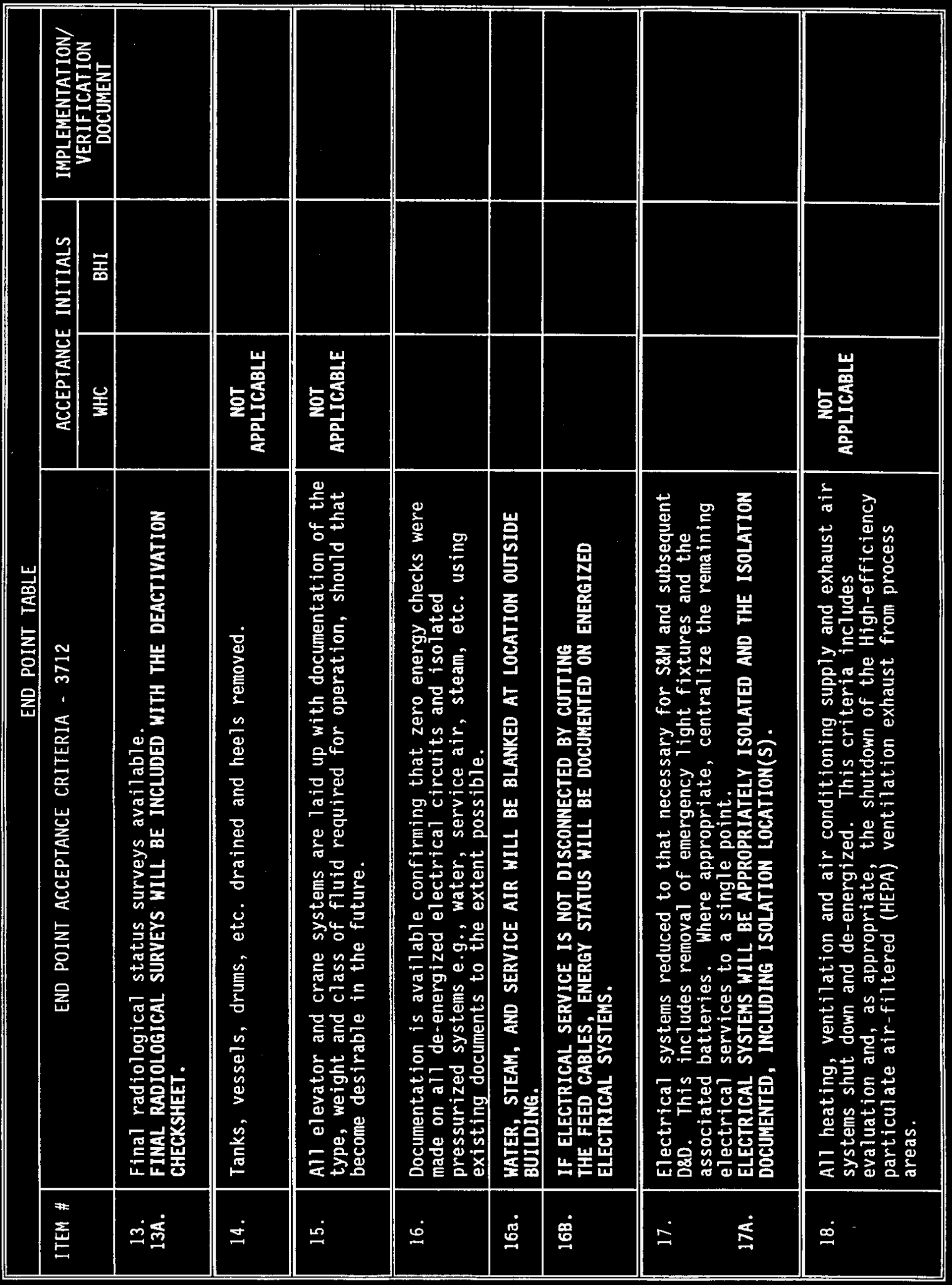


END POINT TABLE

\begin{tabular}{|c|c|c|c|c|}
\hline \multirow[t]{2}{*}{ ITEM \# } & \multirow[t]{2}{*}{ END POINT ACCEPTANCE CRITERIA - 3712} & \multicolumn{2}{|c|}{ ACCEPTANCE INITIALS } & \multirow{2}{*}{$\begin{array}{l}\text { IMPLEMENTATION/ } \\
\text { VERIFICATION } \\
\text { DOCUMENT }\end{array}$} \\
\hline & & WHC & BHI & \\
\hline 19. & A11 unneeded equipment shut down. & $\begin{array}{l}\text { NOT } \\
\text { APPLICABLE }\end{array}$ & & \\
\hline 20. & Fire protection systems downgraded. & & & \\
\hline $20 A$. & ALL PORTABLE FIRE EXTINGUISHERS WILL BE REHOVED FROM THE BUILDING. & & & \\
\hline $20 B$. & $\begin{array}{l}\text { THE FIRE SPRINKLER SYSTEN WILL BE DRAINED AND THE FIRE PROTECTION } \\
\text { RISER WILL BE BLANKED AT THE PIV. }\end{array}$ & & & \\
\hline 21. & $\begin{array}{l}\text { The radiation space monitoring system should be reduced to a level } \\
\text { required by the Health Physics organization. }\end{array}$ & $\begin{array}{l}\text { NOT } \\
\text { APPLICABLE }\end{array}$ & & \\
\hline 22. & $\begin{array}{l}\text { The continuous air monitoring system should be reduced to a level } \\
\text { required by the Health Physics organization. }\end{array}$ & $\begin{array}{l}\text { NOT } \\
\text { APPLICABLE }\end{array}$ & & \\
\hline $\begin{array}{l}23 . \\
23 A .\end{array}$ & $\begin{array}{l}\text { The criticality monitoring and alarm system should be deactivated. } \\
\text { THE CRITICALITY MONITORING AND ALARM SYSTEM WILL BE DEACTIVATED. }\end{array}$ & & & \\
\hline 24. & $\begin{array}{l}\text { Reduce or eliminate the electrical and water supply services to the } \\
\text { building in accordance with the requirements for S\&M. }\end{array}$ & & & \\
\hline $24 A$. & WATER SUPPLY WILL BE BLANKED AT LOCATION OUTSIDE THE BUILDING. & & & \\
\hline 248 . & ELECTRICAL SERVICE WILL BE REDUCED PER ITEM $\# 17$. & & & \\
\hline $\begin{array}{l}25 . \\
25 A\end{array}$ & $\begin{array}{l}\text { Remove a } 11 \text { emergency } 1 \text { ighting and dispose of associated batteries. } \\
\text { ALL EMERGENCY LIGHTING WILL BE RENOVED AND ASSOCIATED BATTERIES } \\
\text { DISPOSED. }\end{array}$ & & & \\
\hline $\begin{array}{l}26 . \\
26 A \text {. }\end{array}$ & $\begin{array}{l}\text { The building steam system should be deactivated and tagged. } \\
\text { STEAN SYSTEM BLANKED AT BUILDING MAIN CONNECTION OUTSIDE THE } \\
\text { BUILDING. }\end{array}$ & & & \\
\hline
\end{tabular}




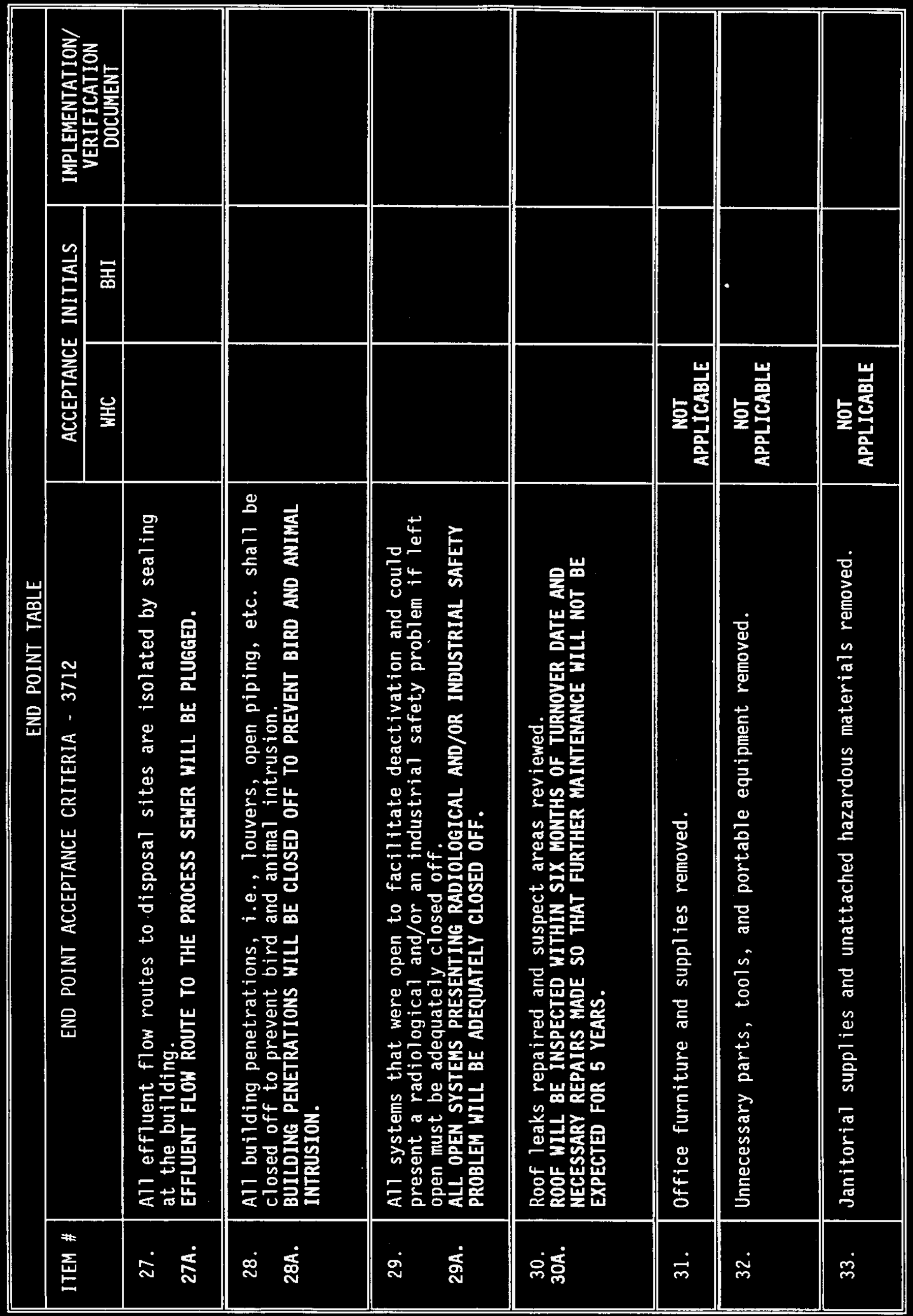




\begin{tabular}{|c|c|c|c|c|}
\hline & & & 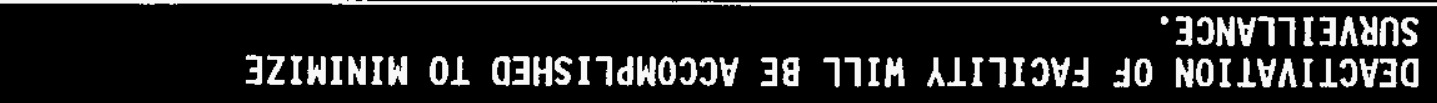 & - $\mathrm{YOt}$ \\
\hline & & & 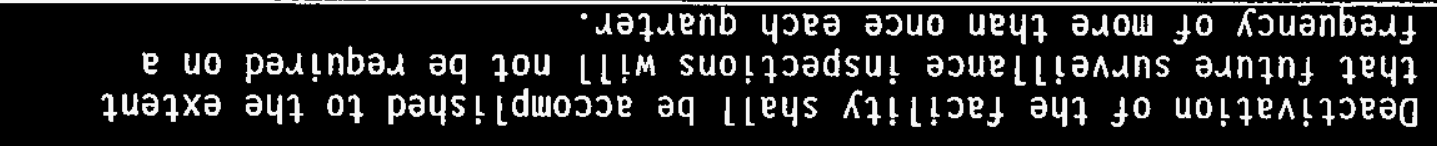 & $\cdot 0 b$ \\
\hline & & $\begin{array}{l}378 Y 9 I 7 d d y \\
10 N\end{array}$ & 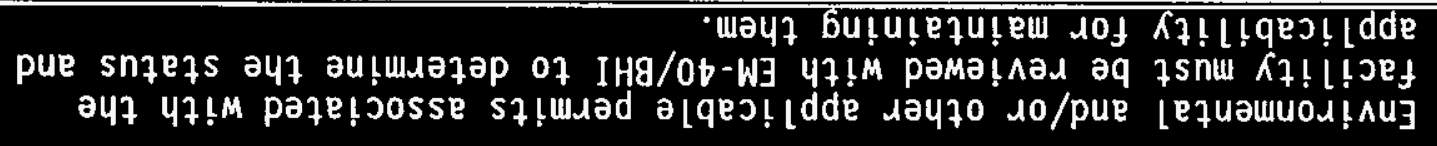 & $\cdot 6 \varepsilon$ \\
\hline & & & 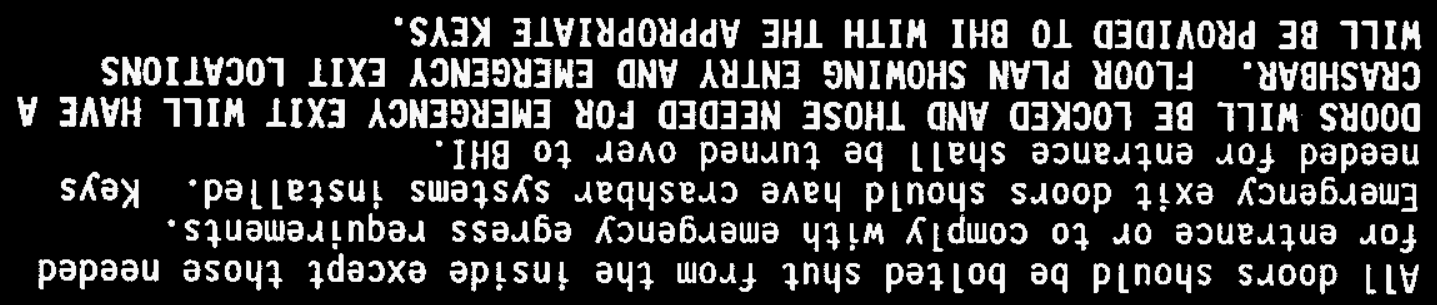 & $\begin{array}{l}\cdot \forall 8 \varepsilon \\
\cdot 8 \varepsilon\end{array}$ \\
\hline & & $\begin{array}{l}\text { 378Y9I7ddy } \\
10 \mathrm{~N}\end{array}$ & 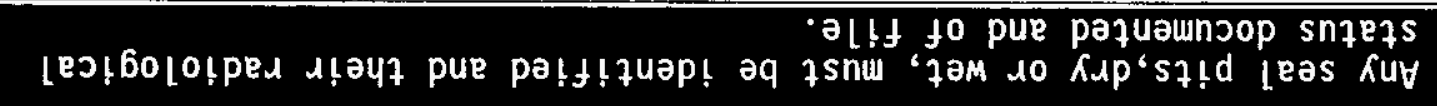 & $\cdot L \varepsilon$ \\
\hline & & $\begin{array}{l}\text { 378yoI Tddy } \\
10 N\end{array}$ & 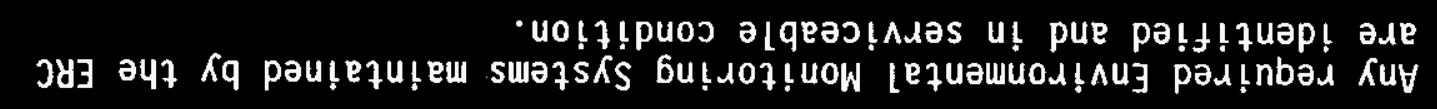 & $\cdot 9 \varepsilon$ \\
\hline & & & 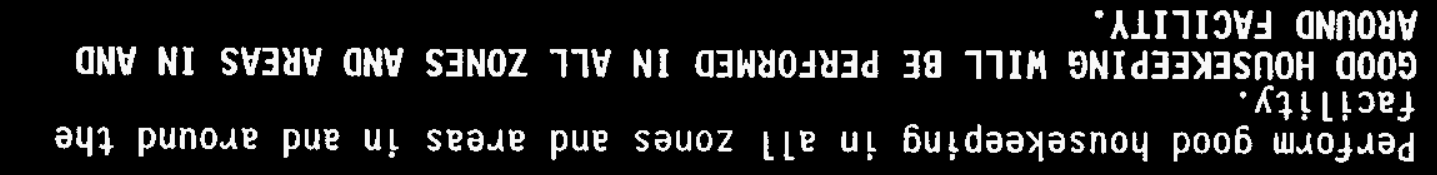 & $\begin{array}{l}\cdot \forall \mathcal{~} 9 \\
\cdot \mathcal{G E}\end{array}$ \\
\hline & & & 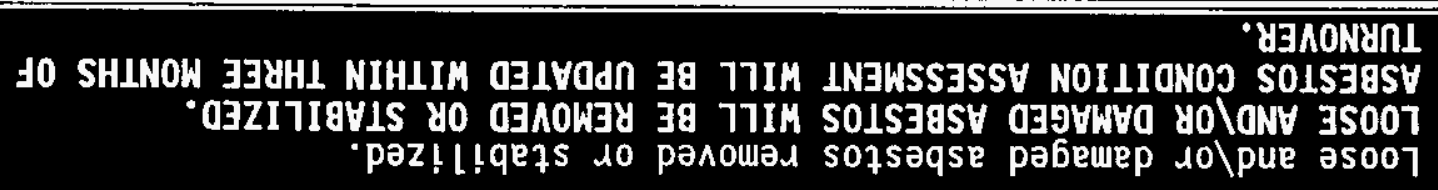 & $\begin{array}{l}\cdot \forall \bullet \varepsilon \\
\cdot \forall \varepsilon\end{array}$ \\
\hline \multirow{2}{*}{ 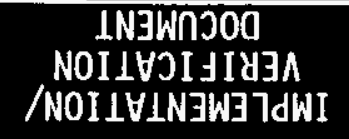 } & IHg & ЈHM & \multirow[b]{2}{*}{ 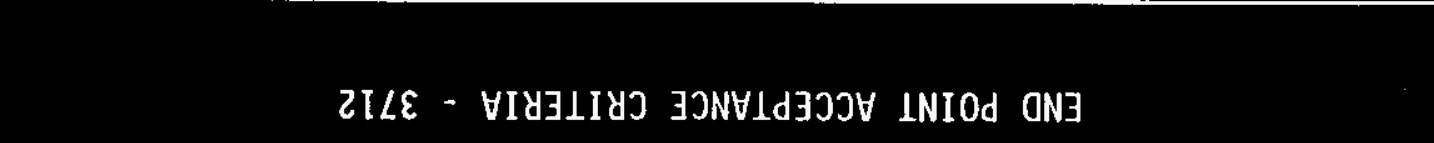 } & \multirow[b]{2}{*}{ \# WJII } \\
\hline & \multicolumn{2}{|c|}{ STVILINI JJNYIdJJJY } & & \\
\hline
\end{tabular}




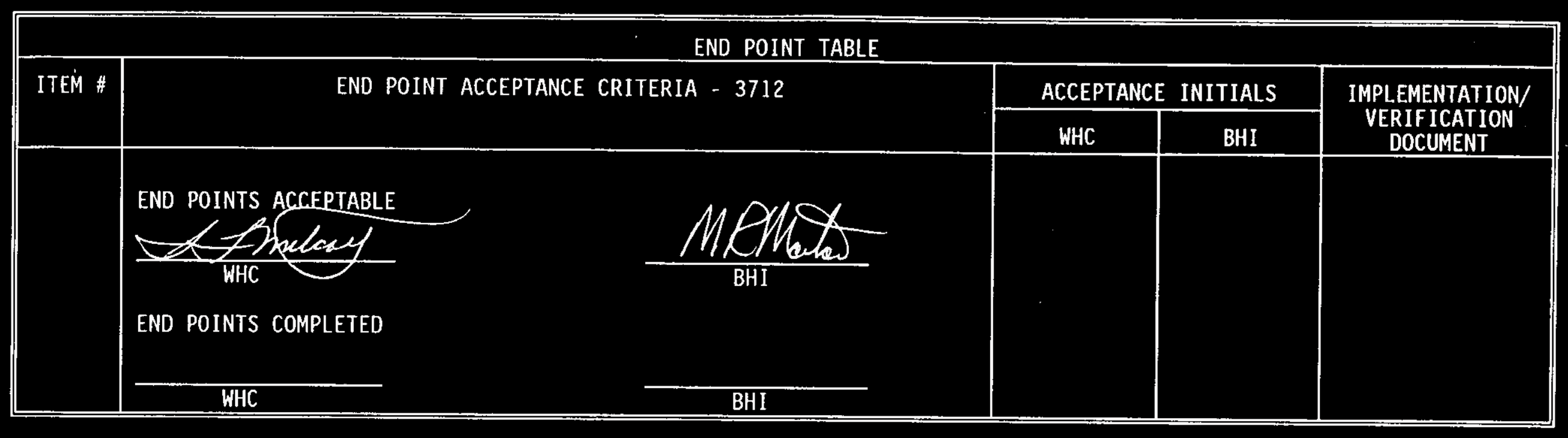




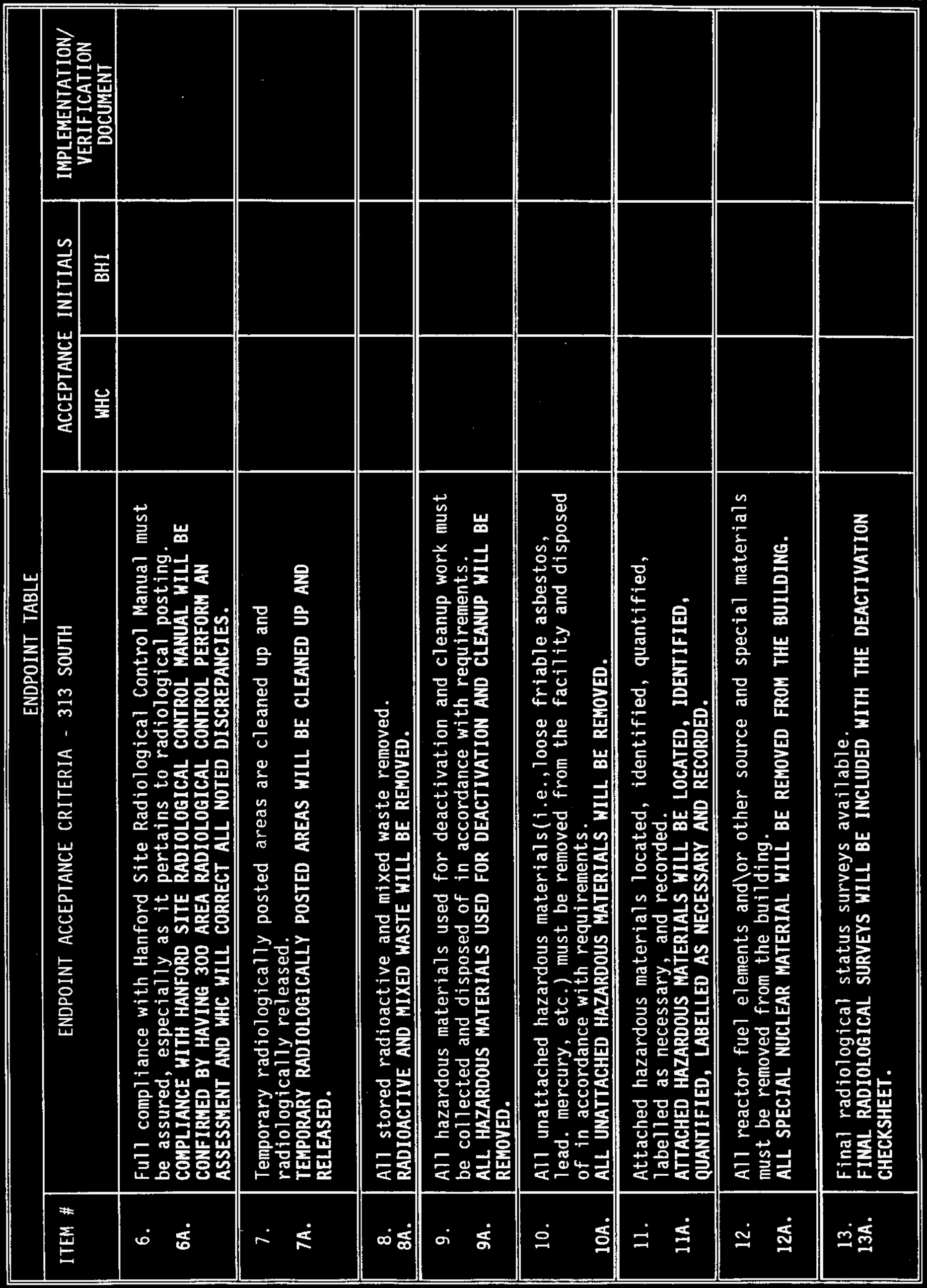


ENDPOINT TABLE

\begin{tabular}{|c|c|c|c|c|}
\hline \multicolumn{5}{|c|}{ ENDPOINT TABLE } \\
\hline \multirow[t]{2}{*}{ ITEM \# } & \multirow{2}{*}{ ENDPOINT ACCEPTANCE CRITERIA - 313 SOUTH } & \multicolumn{2}{|c|}{ ACCEPTANCE INITIALS } & \multirow{2}{*}{$\begin{array}{l}\text { IMPLEMENTATION/ } \\
\text { VERIFICATION } \\
\text { DOCUMENT }\end{array}$} \\
\hline & & WHC & BHI & \\
\hline $\begin{array}{l}14 . \\
14 A .\end{array}$ & $\begin{array}{l}\text { Tanks, vessels, drums, etc. drained and heels removed. } \\
\text { WATS SYSTEM WILL BE CLEAN CLOSED. }\end{array}$ & & & \\
\hline 15. & $\begin{array}{l}\text { All elevator and crane systems are laid up with documentation of the } \\
\text { type, weight and class of fluid required for operation, should that } \\
\text { become desirable in the future. }\end{array}$ & $\begin{array}{l}\text { NOT } \\
\text { APPLICABLE }\end{array}$ & & \\
\hline 16. & $\begin{array}{l}\text { Documentation is available confirming that zero energy checks were } \\
\text { made on all de-energized electrical circuits and isolated } \\
\text { pressurized systems e.g., water, service air, steam, etc. using } \\
\text { existing documents to the extent possible. }\end{array}$ & & & \\
\hline $16 A$. & $\begin{array}{l}\text { WATER, STEAM, AND SERVICE AIR WILL BE BLANKED AT LOCATION OUTSIDE } \\
\text { BUILDING. }\end{array}$ & & & \\
\hline $16 B$. & $\begin{array}{l}\text { IF ELECTRICAL SERVICE IS NOT DISCONNECTED BY CUTTING } \\
\text { THE FEED CABLES, ENERGY STATUS WILL BE DOCUMENTED ON ENERGIZED } \\
\text { ELECTRICAL SYSTEMS. }\end{array}$ & & & \\
\hline $17 A$. & $\begin{array}{l}\text { Electrical systems reduced to that necessary for S\&M and subsequent } \\
\text { D\&D. This includes removal of emergency light fixtures and the } \\
\text { associated batteries. Where appropriate, centralize the remaining } \\
\text { electrical services to a single point. } \\
\text { ELECTRICAL SYSTEMS WILL BE APPROPRIATELY ISOLATED AND THE ISOLATION } \\
\text { DOCUMENTED, INCLUDING ISOLATION LOCATION(S). }\end{array}$ & & & \\
\hline $18 A$. & $\begin{array}{l}\text { A11 heating, ventilation and air conditioning supply and exhaust air } \\
\text { systems shut down and de-energized. This criteria includes } \\
\text { evaluation and, as appropriate, the shutdown of the High-efficiency } \\
\text { particulate air-filtered (HEPA) ventilation exhaust from process } \\
\text { areas. } \\
\text { VENTILATION SYSTEM WILL BE SHUT DOWN AND EXHAUST STACKS CAPPED. }\end{array}$ & & & \\
\hline 19. & A11 unneeded equipment shut down. & $\begin{array}{c}\text { NOT } \\
\text { APPLICABLE }\end{array}$ & & \\
\hline 20. & Fire protection systems downgraded. & & & \\
\hline
\end{tabular}




\begin{tabular}{|c|c|c|c|c|}
\hline \multicolumn{5}{|c|}{ ENDPOINT TABLE } \\
\hline \multirow[t]{2}{*}{ ITEM \# } & \multirow[t]{2}{*}{ ENDPOINT ACCEPTANCE CRITERIA - 313 SOUTH } & \multicolumn{2}{|c|}{ ACCEPTANCE INITIALS } & \multirow{2}{*}{$\begin{array}{l}\text { IMPLEMENTATION } \\
\text { VERIFICATION } \\
\text { DOCUMENT }\end{array}$} \\
\hline & & WHC & BHI & \\
\hline $20 \mathrm{~A}$. & ALL PORTABLE FIRE EXTINGUISHERS UILL BE REYOVED FROM THE BUILDING. & & & \\
\hline $20 \mathrm{~B}$ & THE FIRE ALARM SYSTEM WILL BE DEACTIVATED. & & & \\
\hline 21. & $\begin{array}{l}\text { The radiation space monitoring system should be reduced to a level } \\
\text { required by the Health Physics organization. }\end{array}$ & $\begin{array}{l}\text { NOT } \\
\text { APPLICABLE }\end{array}$ & & \\
\hline 22. & $\begin{array}{l}\text { The continuous air monitoring system should be reduced to a level } \\
\text { required by the Health Physics organization. }\end{array}$ & $\begin{array}{l}\text { NOT } \\
\text { APPLICABLE }\end{array}$ & & \\
\hline $\begin{array}{l}23 . \\
23 A .\end{array}$ & $\begin{array}{l}\text { The criticality monitoring and alarm system should be deactivated. } \\
\text { THE CRITICALITY MONITORING AND ALARM SYSTEM WILL BE DEACTIVATED. }\end{array}$ & & & \\
\hline 24. & $\begin{array}{l}\text { Reduce or eliminate the electrical and water supply services to the } \\
\text { building in accordance with the requirements for S\&M. }\end{array}$ & & & \\
\hline 24A. & WATER SUPPLY WILL BE BLANKED AT LOCATION OUTSIDE THE BUILDING. & & & \\
\hline $24 B$. & ELECTRICAL SERVICE WILL BE REDUCED PER ITEM \#17. & & & \\
\hline 25 & $\begin{array}{l}\text { Remove all emergency } 1 \text { ighting and dispose of associated batteries. } \\
\text { ALL EMERGENCY LIGHTING WILL BE REMOVED AND ASSOCIATED BATTERIES } \\
\text { DISPOSED. }\end{array}$ & & & \\
\hline $\begin{array}{l}26 . \\
26 A\end{array}$ & $\begin{array}{l}\text { The building steam system should be deactivated and tagged. } \\
\text { STEAM BLANKED AT BUILDING WAIN CONNECTION OUTSIDE THE BUILDING. }\end{array}$ & & & \\
\hline 27. & $\begin{array}{l}\text { All effluent flow routes to disposal sites are isolated by sealing } \\
\text { at the building. } \\
\text { EFFLUENT FLOW ROUTES TO THE PROCESS AND SANITARY SEWERS WILL BE } \\
\text { PLUGGED AT THE BUILDING. }\end{array}$ & & & \\
\hline
\end{tabular}




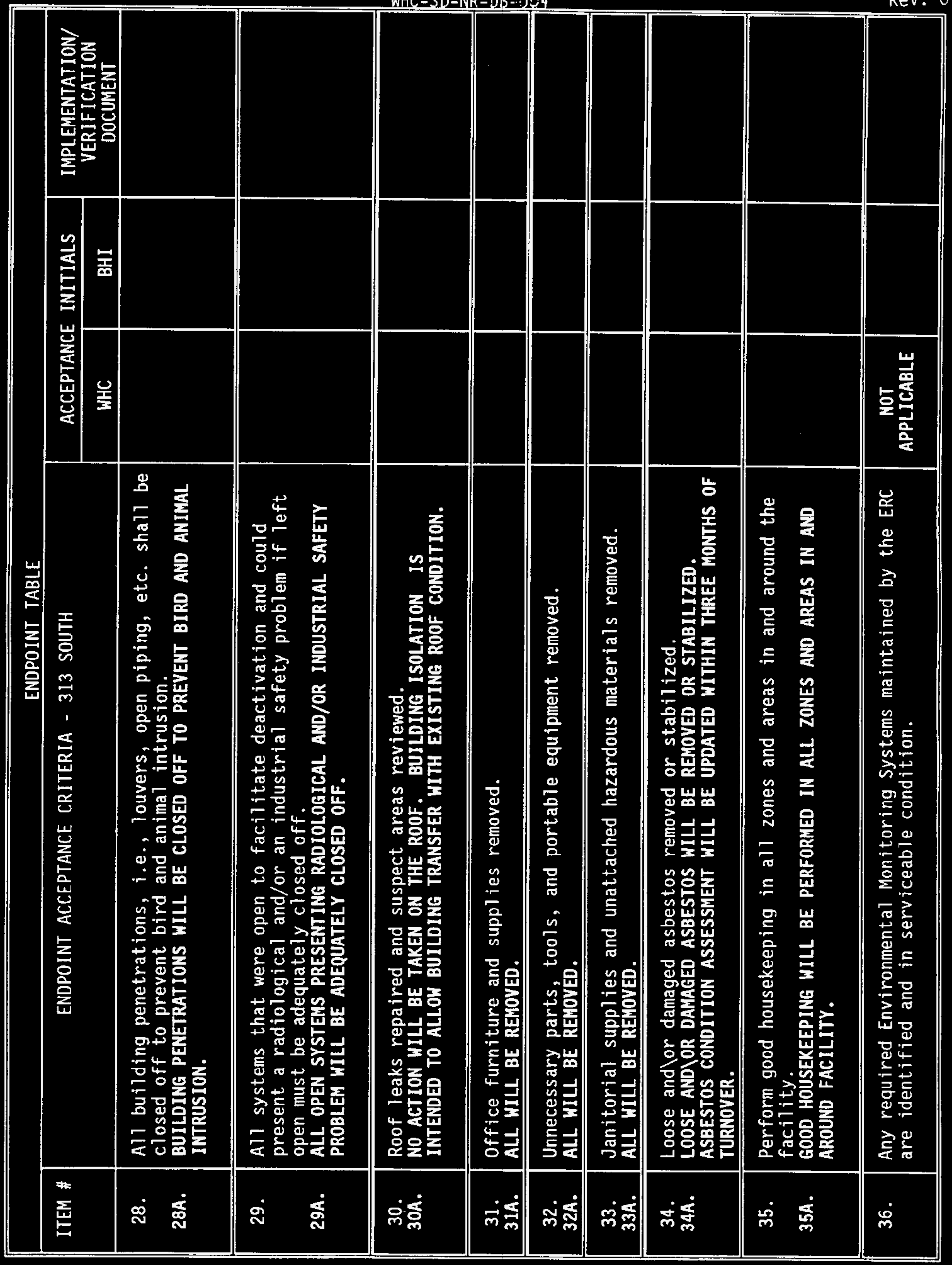




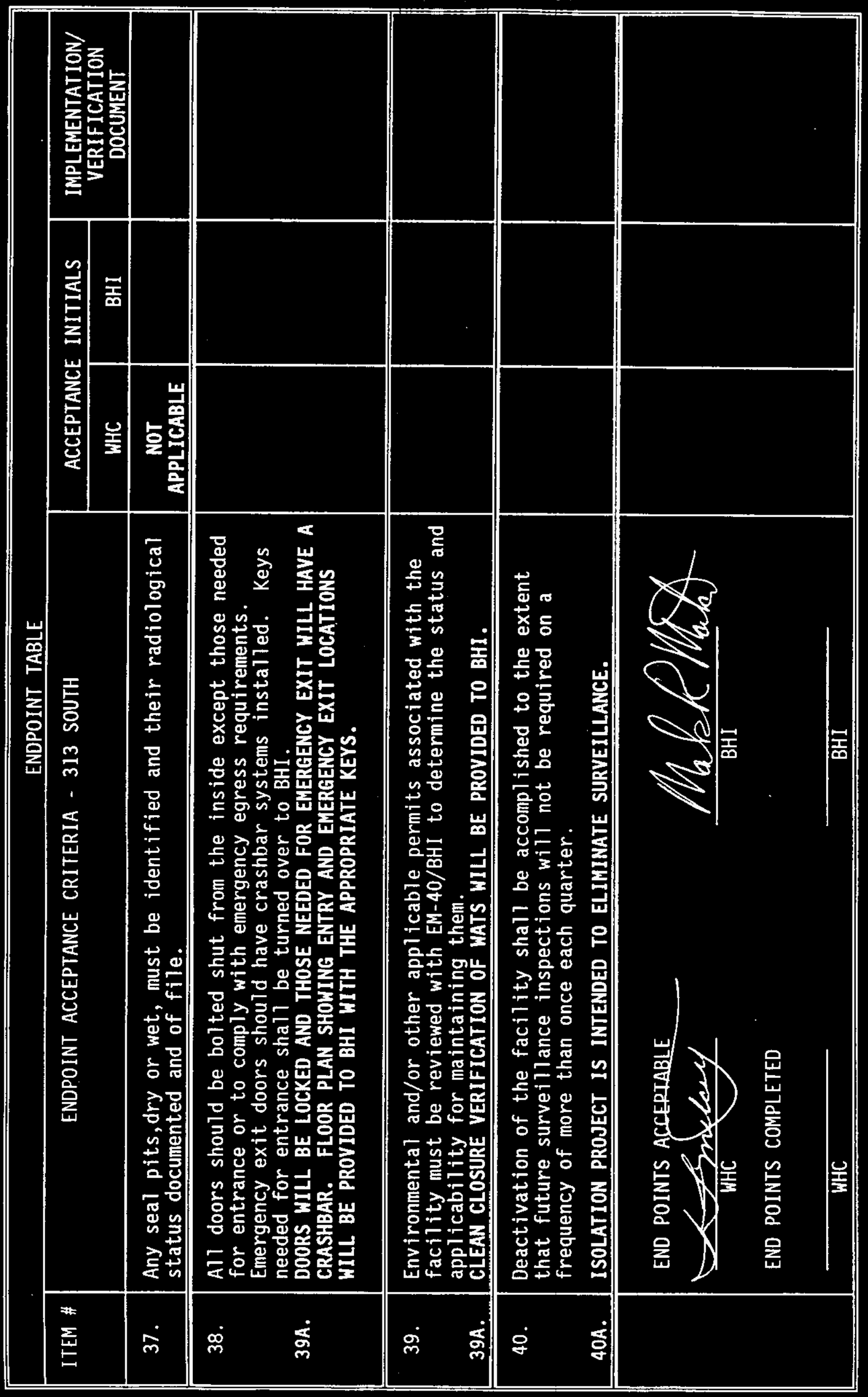




\begin{tabular}{|c|c|c|c|c|}
\hline \multicolumn{5}{|c|}{ ENDPOINT TABLE } \\
\hline \multirow[t]{2}{*}{ ITEM \# } & \multirow[t]{2}{*}{ ENDPOINT ACCEPTANCE CRITERIA - 313 NORTH } & \multicolumn{2}{|c|}{ ACCEPTANCE INITIALS } & \multirow{2}{*}{$\begin{array}{l}\text { IMPLEMENTATION/ } \\
\text { VERIFICATI ION } \\
\text { DOCUMENT }\end{array}$} \\
\hline & & WHC & BHI & \\
\hline 1. & $\begin{array}{l}\text { Prepare and complete deactivation checksheet. } \\
\text { CHECKSHEET WILL BE PREPARED, COMPLETED AND SIGNED OFF BY BOTH } \\
\text { ORGANIZATIONS (WHC AND BHI). }\end{array}$ & & & \\
\hline 2. & $\begin{array}{l}\text { A11 pending Radiation Occurrence reports, Unusual Occurrence Reports } \\
\text { and lor any other out-of-standard condition reports finalized and } \\
\text { closed out in accordance with applicable MRPs. No operable } \\
\text { emergency sirens shall remain mounted on or powered from the } \\
\text { building. }\end{array}$ & & & \\
\hline $2 \mathrm{~A}$. & $\begin{array}{l}\text { RADIATION OCCURRENCE REPORTS, UNUSUAL OCCURRENCE REPORTS ANDJOR ANY } \\
\text { OTHER OUT-OF-STANDARD CONDITIONS REPORTS WILL BE FINALIZED AND } \\
\text { CLOSED BY WHC. }\end{array}$ & & & \\
\hline 3. & $\begin{array}{l}\text { The Fuel Supply Shutdown Facilities Interim Safety Basis Report and } \\
\text { Plant Emergency Procedures shall be reviewed and updated for } \\
\text { deactivation/shutdown status. }\end{array}$ & & & \\
\hline 3A. & $\begin{array}{l}\text { REVIEW AND UPDATE OF THE INTERIM SAFETY BASIS REPORT WILL BE } \\
\text { COMPLETED AND PROVIDED TO BHI } 6 \text { MONTHS PRIOR TO TURNOVER. }\end{array}$ & & & \\
\hline 3B. & $\begin{array}{l}\text { REVIEN AND UPDATE OF THE PLANT ENERGENCY PROCEDURES YILL BE } \\
\text { COMPLETED AND PROVIDED TO BHI } 6 \text { MONTHS PRIOR TO TURNOVER. }\end{array}$ & & & \\
\hline 4 4. & $\begin{array}{l}\text { CVI index, drawings, procedures, prints, photographs, history, } \\
\text { design and operating documents provided. } \\
\text { AVALABLE ESSENIAL DRANINGS, A DRAWING LIST, PROCEDURES, ETC. WILL } \\
\text { BE PROVIDED IN TURNOVER FILE. }\end{array}$ & & & \\
\hline 5. & $\begin{array}{l}\text { All permanent radiologically contaminated areas to be decontaminated } \\
\text { and released or the surface contamination levels reduced or fixed in } \\
\text { place to minimize re-suspension and } \backslash \text { or migration of loose } \\
\text { Contamination. } \\
\text { ANY CONTAMINATED ZONES WILL BE DECONTAMINATED AND RELEASED OR } \\
\text { CONTAMINATION LEVELS REDUCED AND FIXED IN PLACE. }\end{array}$ & & & \\
\hline
\end{tabular}




\begin{tabular}{|c|c|c|c|c|}
\hline \multicolumn{5}{|c|}{ ENDPOINT TABLE } \\
\hline \multirow[t]{2}{*}{ ITEM \# } & \multirow[t]{2}{*}{ ENDPOINT ACCEPTANCE CRITERIA - 313 NORTH } & \multicolumn{2}{|c|}{ ACCEPTANCE INITIALS } & \multirow{2}{*}{$\begin{array}{l}\text { IMPLEMENTATION/ } \\
\text { VERIFICATION } \\
\text { DOCUMENT }\end{array}$} \\
\hline & & HHC & BHI & \\
\hline $\begin{array}{l}6 . \\
6 A .\end{array}$ & $\begin{array}{l}\text { Ful1 compliance with Hanford Site Radiological Control Manual must } \\
\text { be assured, especially as it pertains to radiological posting. } \\
\text { COMPLIANCE WITH HANFORD SITE RADIOLOGICAL CONTROL WANUAL WILL BE } \\
\text { CONFIRMED BY HAVING 300 AREA RADIOLOGICAL CONTROL PERFORH AN } \\
\text { ASSESSMENT AND WHC WILL CORRECT ALL NOTED DISCREPANCIES. }\end{array}$ & & & \\
\hline 7. & $\begin{array}{l}\text { Temporary radiologically posted areas are cleaned up and } \\
\text { radiologically released. } \\
\text { TEMPORARY RADIOLOGICALLY POSTED AREAS WILL BE CLEANED UP AND } \\
\text { RELEASED. }\end{array}$ & & & \\
\hline 8. & $\begin{array}{l}\text { A11 stored radioactive and mixed waste removed. } \\
\text { RADIOACTIVE AND MIXED WASTE WILL BE REMOVED. }\end{array}$ & & & \\
\hline 9. & $\begin{array}{l}\text { All hazardous materials used for deactivation and cleanup work must } \\
\text { be collected and disposed of in accordance with requirements. } \\
\text { ALL HAZARDOUS MATERIALS USED FOR DEACTIVATION AND CLEANUP WILL BE } \\
\text { REMOVED. }\end{array}$ & & & \\
\hline 10. & $\begin{array}{l}\text { A11 unattached hazardous materials (i.e., loose friable asbestos, } \\
\text { lead. mercury, etc.) must be removed from the facility and disposed } \\
\text { of in accordance with requirements. } \\
\text { ALL UNATTACHED HAZARDOUS MATERIALS WILL BE REMOVED. }\end{array}$ & & & \\
\hline $\begin{array}{l}11 . \\
11 A .\end{array}$ & $\begin{array}{l}\text { Attached hazardous materials located, identified, quantified, } \\
\text { labelled as necessary, and recorded. } \\
\text { ATTACHED HAZARDOUS MATERIALS WILL BE LOCATED, IDENTIFIED, } \\
\text { QUANTIFIED, LABELLED AS NECESSARY AND RECORDED. }\end{array}$ & & & \\
\hline 12. & $\begin{array}{l}\text { Al1 reactor fuel elements and } \backslash \text { or other source and special materials } \\
\text { must be removed from the building. } \\
\text { ALL SPECIAL NUCLEAR MATERIAL WILL BE REMOVED FROH THE BUILDING. }\end{array}$ & & & \\
\hline $\begin{array}{l}13 . \\
13 A\end{array}$ & $\begin{array}{l}\text { Final radiological status surveys available. } \\
\text { FINAL RADIOLOGICAL SURVEYS WILL BE INCLUDED WITH THE DEACTIVATION } \\
\text { CHECKSHEET. }\end{array}$ & & & \\
\hline
\end{tabular}




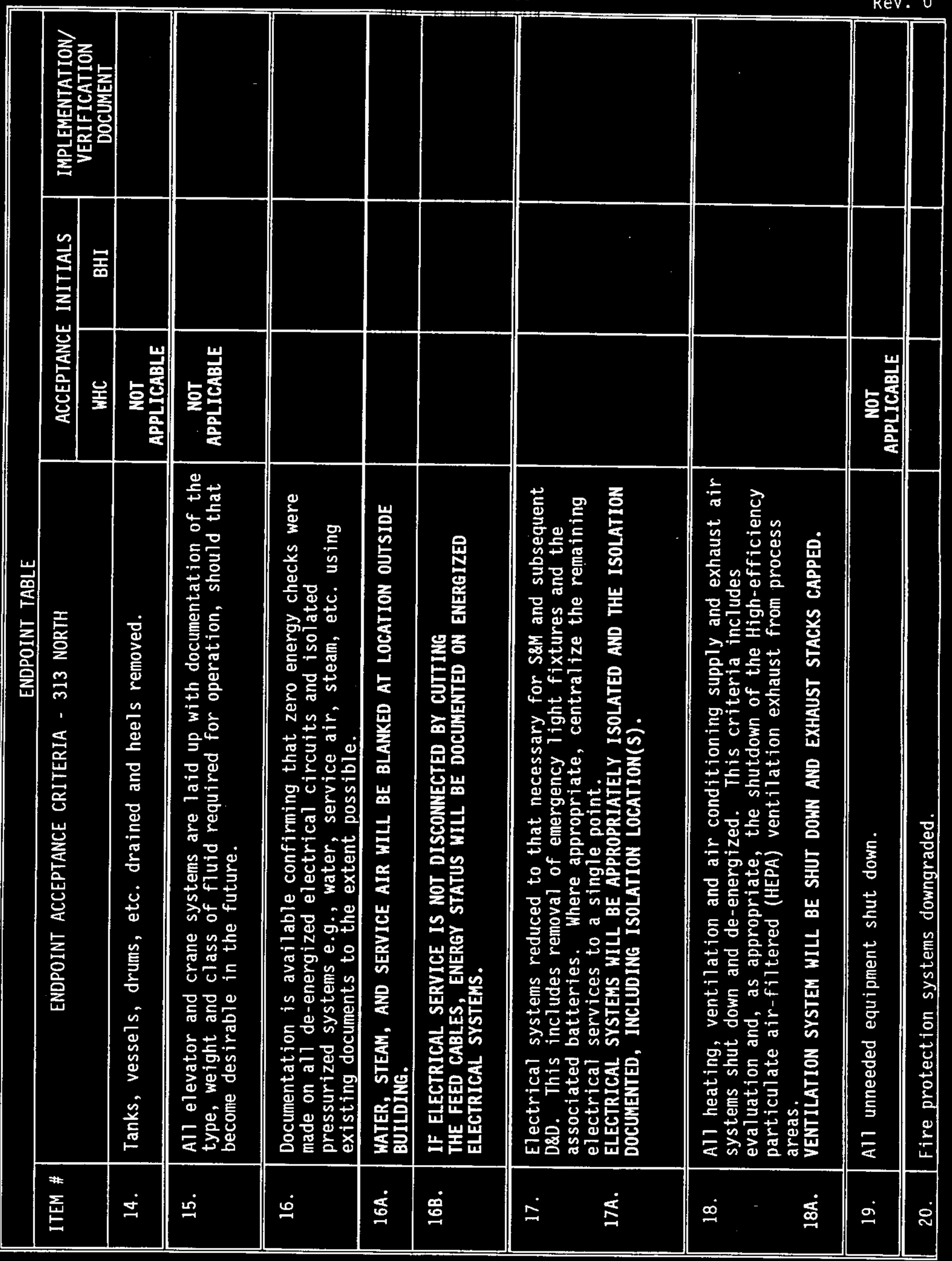




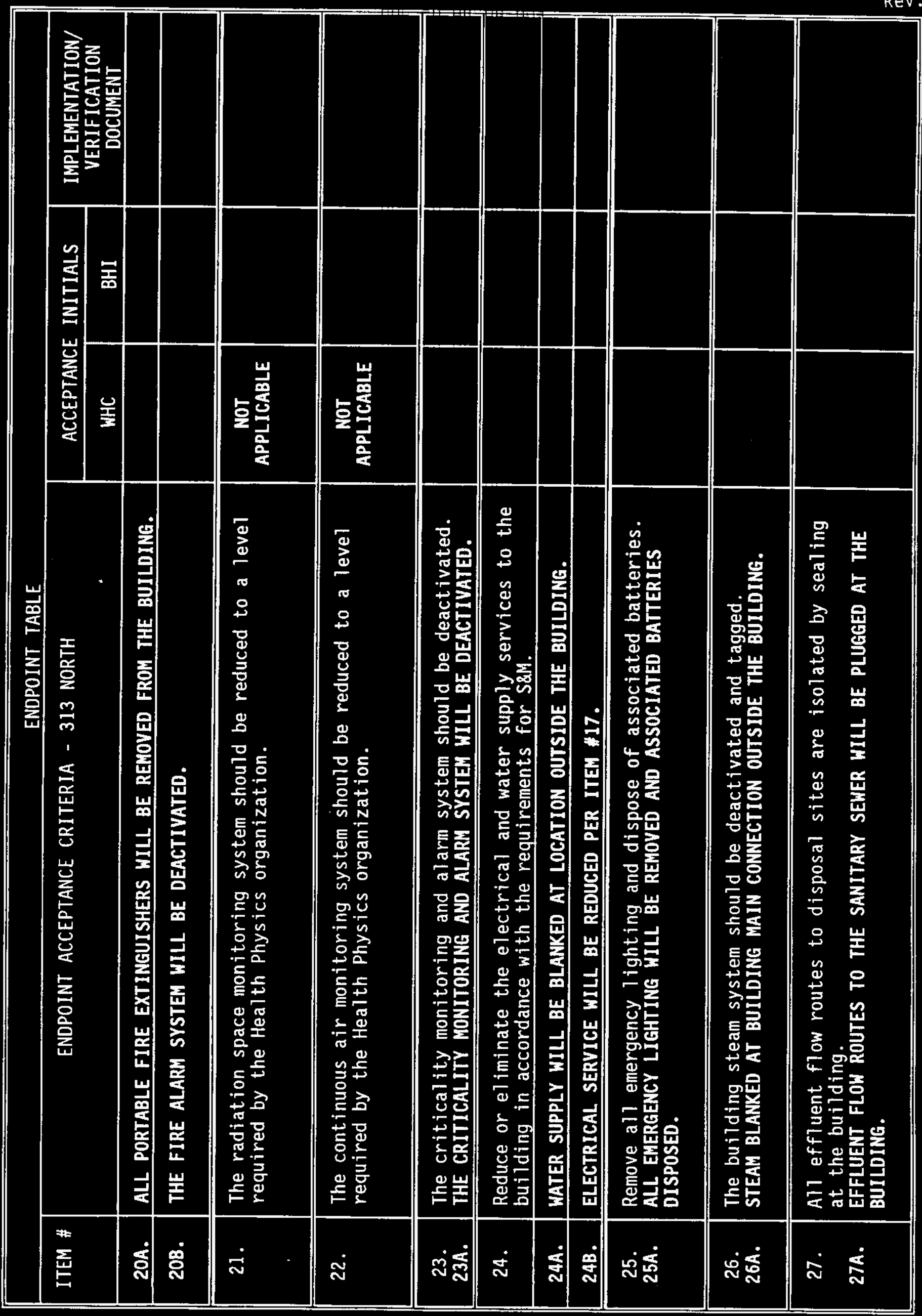




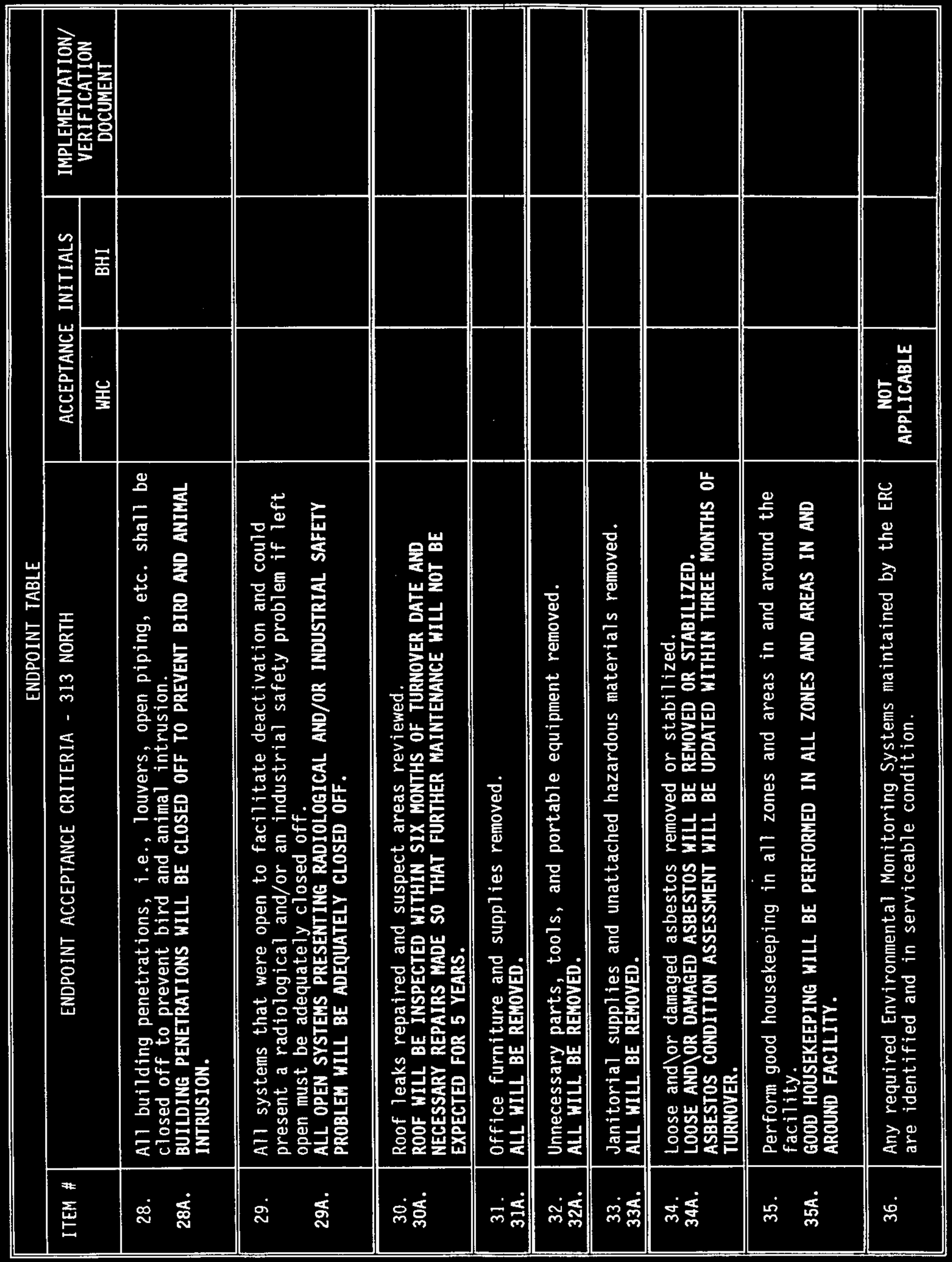




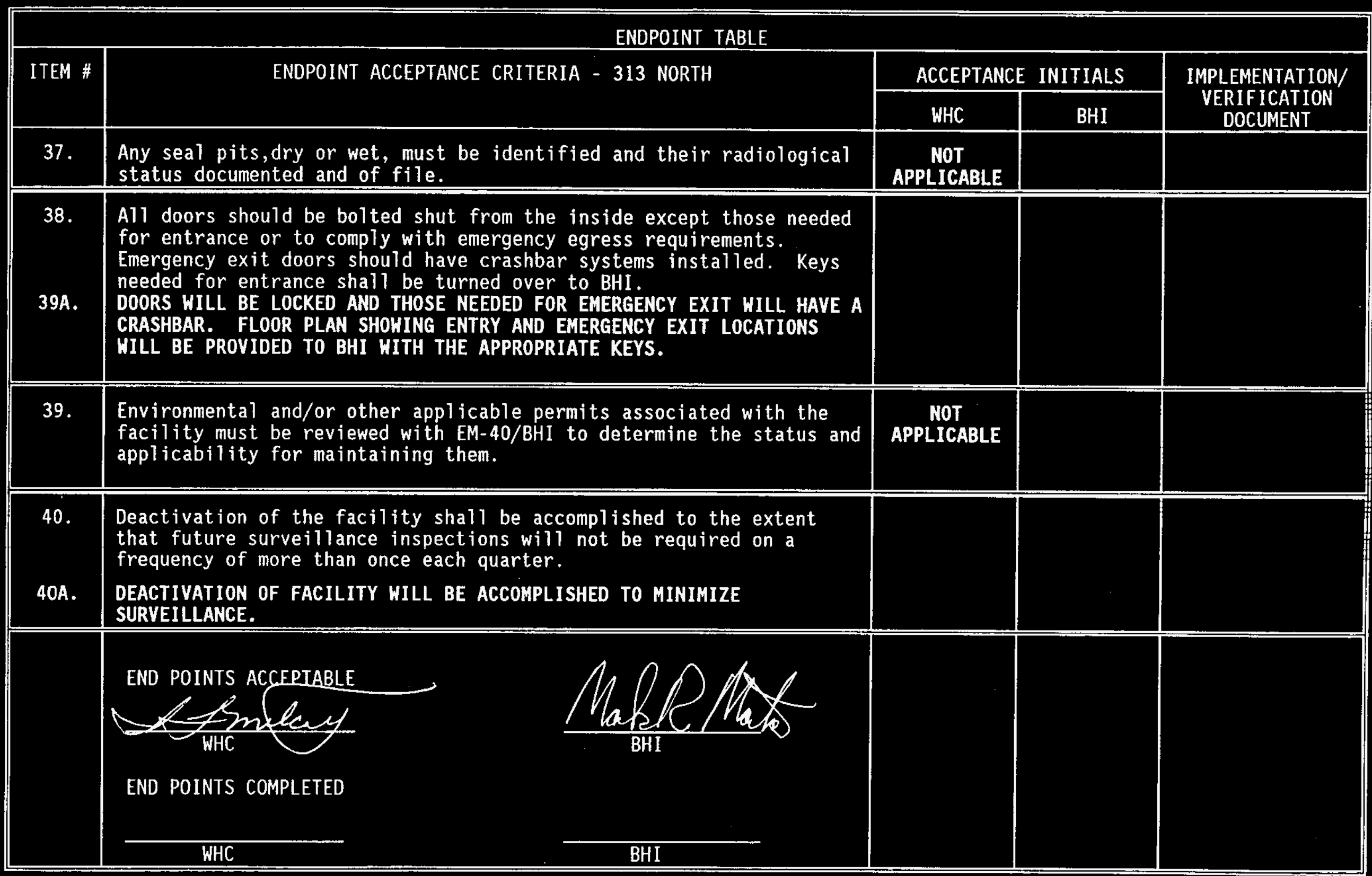

$\underset{0}{\stackrel{D}{\mathbb{D}}}$

$\stackrel{D}{\infty}$ 


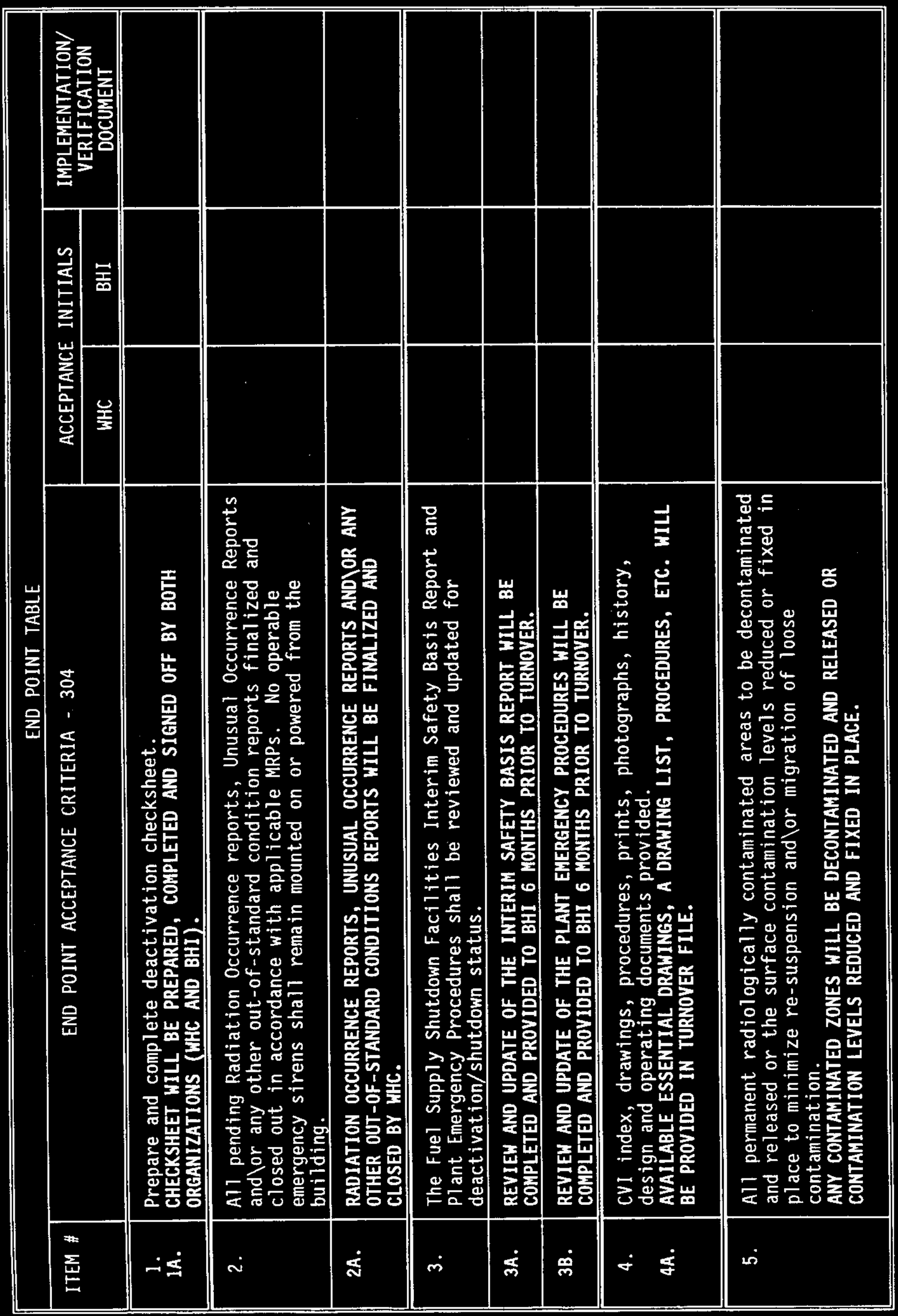


$A^{-}-2-3-1 x_{-}-1-u^{4}$

Rev. 0

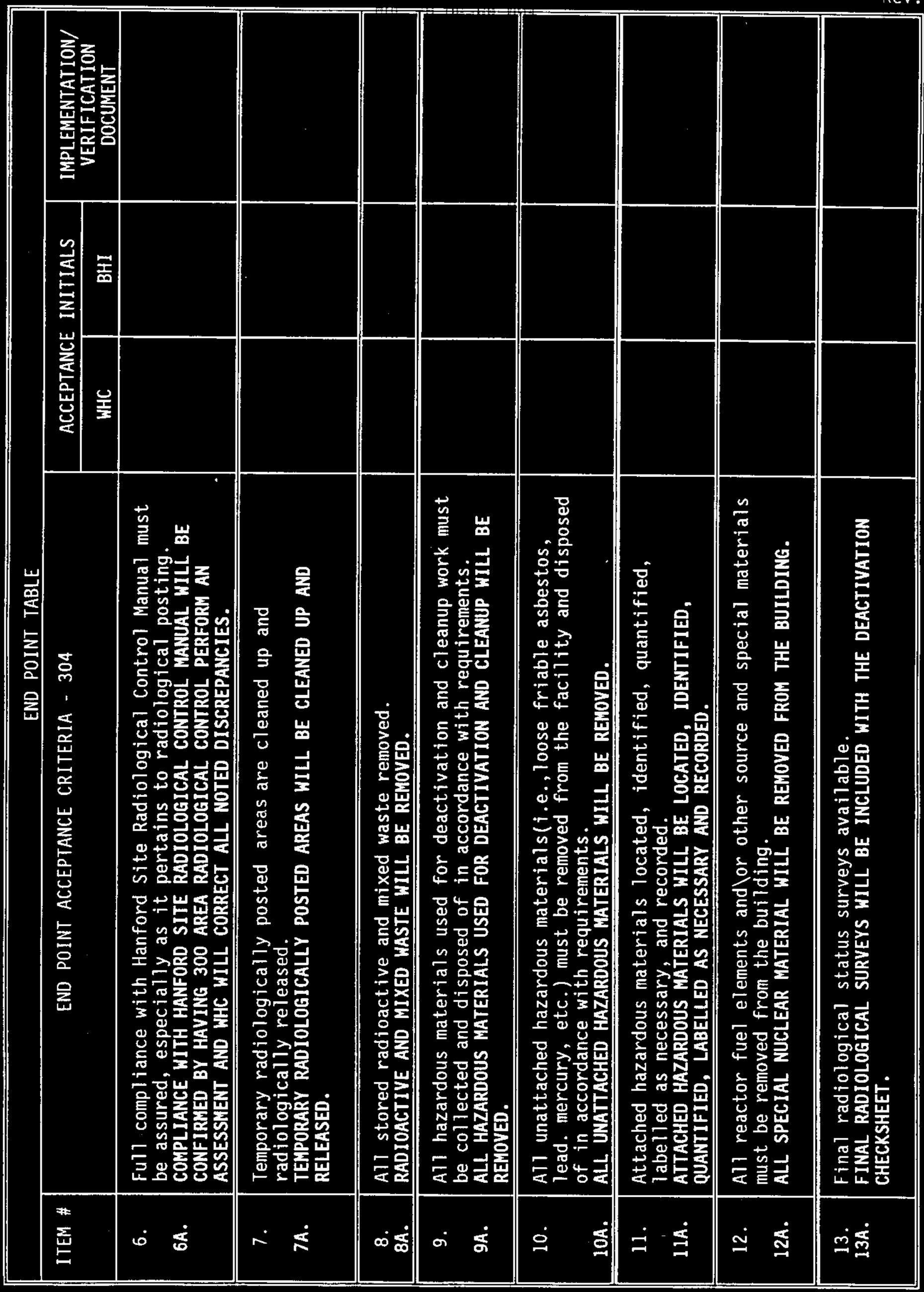


WHC-SD-NR-DE-004

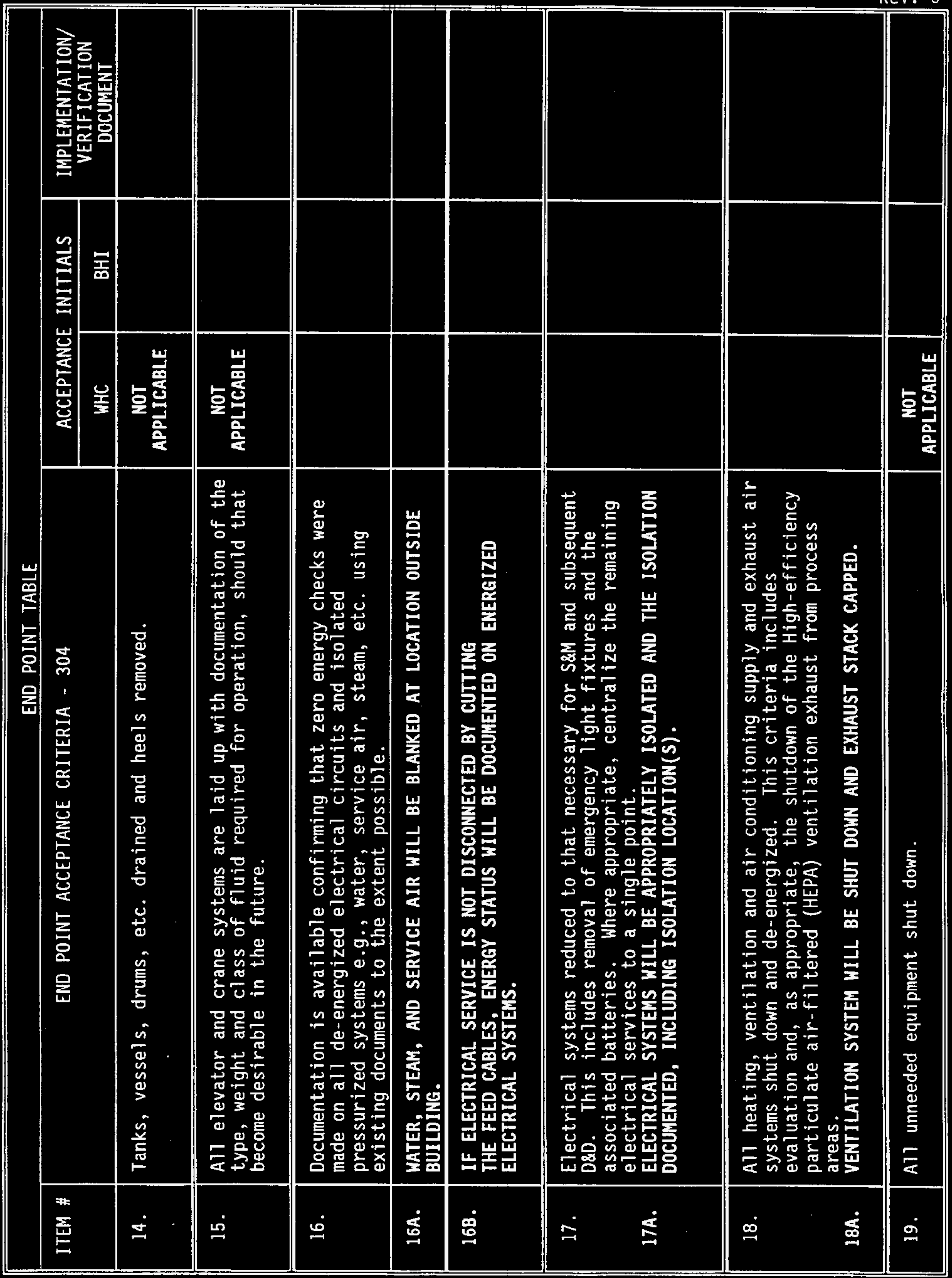


WHC-SD-NR-DB-004

Rev. 0

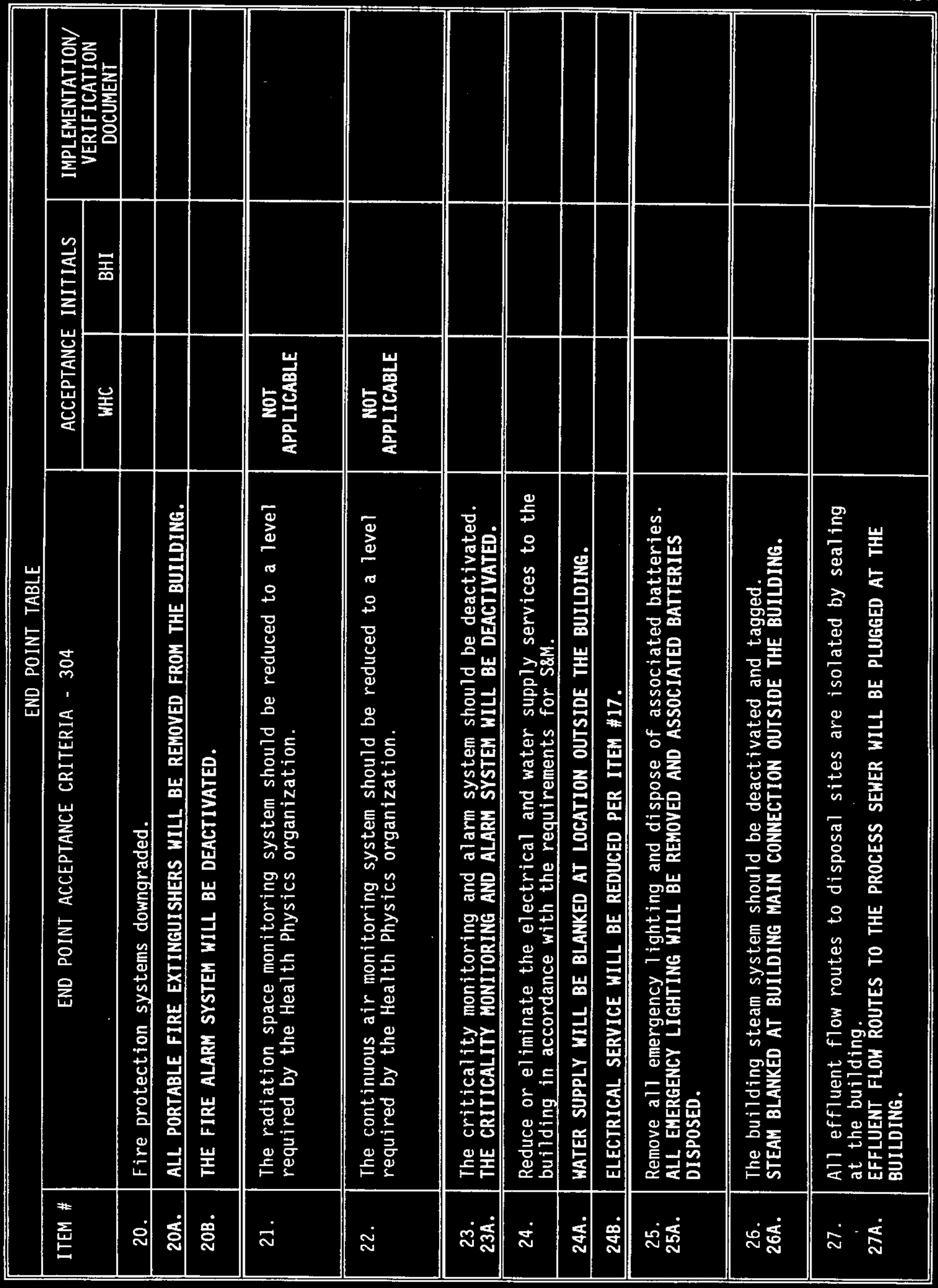




\begin{tabular}{|c|c|c|c|c|}
\hline \multicolumn{5}{|c|}{ END POINT TABLE } \\
\hline \multirow[t]{2}{*}{ ITEM \# } & \multirow[t]{2}{*}{ END POINT ACCEPTANCE CRITERIA - 304} & \multicolumn{2}{|c|}{ ACCEPTANCE INITIALS } & \multirow{2}{*}{$\begin{array}{l}\text { IMPLEMENTATION/ } \\
\text { VERIFICATION } \\
\text { DOCUMENT }\end{array}$} \\
\hline & & WHC & BHI & \\
\hline 28. & $\begin{array}{l}\text { Al1 building penetrations, i.e., louvers, open piping, etc. shall be } \\
\text { closed off to prevent bird and animal intrusion. } \\
\text { BUILDING PENETRATIONS WILL BE CLOSED OFF TO PREVENT BIRD AND ANIMAL } \\
\text { INTRUSION. }\end{array}$ & & & \\
\hline 29. & $\begin{array}{l}\text { A11 systems that were open to facilitate deactivation and could } \\
\text { present a radiological and/or an industrial safety problem if left } \\
\text { open must be adequately closed off. } \\
\text { ALL OPEN SYSTEMS PRESENTING RADIOLOGICAL AND/OR INDUSTRIAL SAFETY } \\
\text { PROBLEM WILL BE ADEQUATELY CLOSED OFF. }\end{array}$ & & & \\
\hline $\begin{array}{l}30 \\
30 A\end{array}$ & $\begin{array}{l}\text { Roof leaks repaired and suspect areas reviewed. } \\
\text { ROOF WILL BE INSPECTED WITHIN SIX WONTHS OF TURNOVER DATE AND } \\
\text { NECESSARY REPAIRS MADE SO THAT FURTHER MAINTENANCE WILL NOT BE } \\
\text { EXPECTED FOR } 5 \text { YEARS. }\end{array}$ & & & \\
\hline 31. & Office furniture and supplies removed. & $\begin{array}{c}\text { NOT } \\
\text { APPLICABLE }\end{array}$ & & \\
\hline $\begin{array}{l}32 . \\
32 A .\end{array}$ & $\begin{array}{l}\text { Unnecessary parts, tools, and portable equipment removed. } \\
\text { ALL WILL BE REMOVED. }\end{array}$ & & & \\
\hline 33 . & Janitorial supplies and unattached hazardous materials removed. & $\begin{array}{l}\text { NOT } \\
\text { APPLICABLE }\end{array}$ & & \\
\hline $\begin{array}{l}34 . \\
344 .\end{array}$ & $\begin{array}{l}\text { Loose and \or damaged asbestos removed or stabilized. } \\
\text { LOOSE AND \OR DAMAGED ASBESTOS WILL BE RENOVED OR STABILIZED. } \\
\text { ASBESTOS CONDITION ASSESSMENT WILL BE UPDATED WITHIN THREE MONTHS OF } \\
\text { TURNOVER. }\end{array}$ & & & \\
\hline 35. & $\begin{array}{l}\text { Perform good housekeeping in all zones and areas in and around the } \\
\text { facility. } \\
\text { GOOD HOUSEKEEPING WILL BE PERFORMED IN ALL ZONES AND AREAS IN AND } \\
\text { AROUND FACILITY. }\end{array}$ & & & \\
\hline
\end{tabular}




\begin{tabular}{|c|c|c|c|c|}
\hline \multicolumn{5}{|c|}{ END POINT TABLE } \\
\hline \multirow[t]{2}{*}{ ITEM \# } & \multirow[t]{2}{*}{ END POINT ACCEPTANCE CRITERIA - 304} & \multicolumn{2}{|c|}{ ACCEPTANCE INITIALS } & \multirow{2}{*}{$\begin{array}{l}\text { IMPLEMENTATION } \\
\text { VERIFICATION } \\
\text { DOCUMENT }\end{array}$} \\
\hline & & WHC & BHI & \\
\hline 36. & $\begin{array}{l}\text { Any required Environmental Monitoring Systems maintained by the ERC } \\
\text { are identified and in serviceable condition. }\end{array}$ & $\begin{array}{l}\text { NOT } \\
\text { APPLICABLE }\end{array}$ & & \\
\hline 37. & $\begin{array}{l}\text { Any seal pits,dry or wet, must be identified and their radiological } \\
\text { status documented and of file. }\end{array}$ & $\begin{array}{l}\text { NOT } \\
\text { APPLICABLE }\end{array}$ & & \\
\hline $39 A$. & $\begin{array}{l}\text { All doors should be bolted shut from the inside except those needed } \\
\text { for entrance or to comply with emergency egress requirements. } \\
\text { Emergency exit doors should have crashbar systems installed. Keys } \\
\text { needed for entrance shall be turned over to BHI. } \\
\text { DOORS WILL BE LOCKED AND THOSE. NEEDED FOR ENERGENCY EXIT WILL HAVE A } \\
\text { CRASHBAR. FLOOR PLAN SHOWING ENTRY AND ENERGENCY EXIT LOCATIONS } \\
\text { WILL BE PROVIDED TO BHI WITH THE APPROPRIATE KEYS. }\end{array}$ & & & \\
\hline $\begin{array}{l}39 . \\
39 A .\end{array}$ & $\begin{array}{l}\text { Environmental and/or other applicable permits associated with the } \\
\text { facility must be reviewed with EM-40/BHI to determine the status and } \\
\text { applicability for maintain ing them. } \\
\text { CLEAN CLOSURE VERIFICATION WILL BE PROVIDED TO BHI. }\end{array}$ & & & \\
\hline 40. & $\begin{array}{l}\text { Deactivation of the facility shall be accomplished to the extent } \\
\text { that future surveillance inspections will not be required on a } \\
\text { frequency of more than once each quarter. }\end{array}$ & & & \\
\hline \multirow[t]{3}{*}{ 40A. } & $\begin{array}{l}\text { DEACTIVATION OF FACILITY WILL BE ACCOMPLISHED TO MINIMIZE } \\
\text { SURVEILLANCE. }\end{array}$ & & & \\
\hline & END POINTS ACCEPTABLE & & & \\
\hline & WHC & & & \\
\hline
\end{tabular}




\begin{tabular}{|c|c|c|c|c|}
\hline \multicolumn{5}{|c|}{ END POINT TABLE } \\
\hline \multirow[t]{2}{*}{ ITEM \# } & \multirow[t]{2}{*}{ END POINT ACCEPTANCE CRITERIA - 334, 334A } & \multicolumn{2}{|c|}{ ACCEPTANCE INITIALS } & \multirow{2}{*}{$\begin{array}{l}\text { IMPLEMENTATION/ } \\
\text { VERIFICATION } \\
\text { DOCUMENT } \\
\end{array}$} \\
\hline & & WHC & $\mathrm{BHI}$ & \\
\hline 1. & $\begin{array}{l}\text { Prepare and complete deactivation checksheet. } \\
\text { CHECKSHEET WILL BE PREPARED, COMPLETED AND SIGNED OFF BY BOTH } \\
\text { ORGANIZATIONS (WHC AND BHI). }\end{array}$ & & & \\
\hline 2. & $\begin{array}{l}\text { A11 pending Radiation Occurrence reports, Unusual Occurrence Reports } \\
\text { and } \backslash \text { or any other out-of-standard condition reports finalized and } \\
\text { closed out in accordance with applicable MRPs. No operable } \\
\text { emergency sirens shall remain mounted on or powered from the } \\
\text { building. }\end{array}$ & & & \\
\hline $2 A$. & $\begin{array}{l}\text { RADIATION OCCURRENCE REPORTS, UNUSUAL OCCURRENCE REPORTS AND \OR ANY } \\
\text { OTHER OUT-OF-STANDARD CONDITIONS REPORTS WILL BE FINALIZED AND } \\
\text { CLOSED BY WHC. }\end{array}$ & & & \\
\hline 3. & $\begin{array}{l}\text { The Fuel Supply Shutdown Facilities Interim Safety Bas is Report and } \\
\text { Plant Emergency Procedures shall be reviewed and updated for } \\
\text { deactivation/shutdown status. }\end{array}$ & & & \\
\hline 3A. & $\begin{array}{l}\text { REVIEN AND UPDATE OF THE INTERIM SAFETY BASIS REPORT WILL BE } \\
\text { CONPLETED AND PROVIDED TO BHI } 6 \text { MONTHS PRIOR TO TURNOVER. }\end{array}$ & & & \\
\hline 3B. & $\begin{array}{l}\text { REVIEN AND UPDATE OF THE PLANT EMERGENCY PROCEDURES UILL BE } \\
\text { CONPLETED AND PROVIDED TO BHI } 6 \text { MONTHS PRIOR TO TURNOVER. }\end{array}$ & & & \\
\hline 4 . & $\begin{array}{l}\text { CVI index, drawings, procedures, prints, photographs, history, } \\
\text { design and operating documents provided. } \\
\text { AVAILABLE ESSENTIAL DRAWINGS, A DRAWING LIST, PROCEDURES, ETC. WILL } \\
\text { BE PROVIDED IN TURNOVER FILE. }\end{array}$ & & & \\
\hline $5 A$. & $\begin{array}{l}\text { A11 permanent radiologically contaminated areas to be decontaminated } \\
\text { and released or the surface contamination levels reduced or fixed in } \\
\text { place to minimize re-suspension and } \backslash \text { or migration of loose } \\
\text { contamination. } \\
\text { CONTAMINATED ZONES WILL BE DECONTAMINATED AND RELEASED OR } \\
\text { CONTAMINATION LEVELS REDUCED AND FIXED IN PLACE. }\end{array}$ & & & \\
\hline
\end{tabular}




\begin{tabular}{|c|c|c|c|c|}
\hline \multicolumn{5}{|c|}{ END POINT TABLE } \\
\hline \multirow[t]{2}{*}{ ITEM \# } & \multirow[t]{2}{*}{ END POINT ACCEPTANCE CRITERIA - $334,334 \mathrm{~A}$} & \multicolumn{2}{|c|}{ ACCEPTANCE INITIALS } & \multirow{2}{*}{$\begin{array}{l}\text { IMPLEMENTATION/ } \\
\text { VERIFICATION } \\
\text { DOCUMENT }\end{array}$} \\
\hline & & WHC & BHI & \\
\hline 6. & $\begin{array}{l}\text { Full compliance with Hanford Site Radiological Control Manual must } \\
\text { be assured, especially as it pertains to radiological posting. } \\
\text { COMPLIANCE KITH HANFORD SITE RADIOLOGICAL CONTROL MANUAL WILL BE } \\
\text { CONFIRMED BY HAVING } 300 \text { AREA RADIOLOGICAL CONTROL PERFORM AN } \\
\text { ASSESSMENT AND WHC WILL CORRECT ALL NOTED DISCREPANCIES. }\end{array}$ & & & \\
\hline 7 . & $\begin{array}{l}\text { Temporary radiologically posted areas are cleaned up and } \\
\text { radiologically released. } \\
\text { TEMPORARY RADIOLOGICALLY POSTED AREAS WILL BE CLEANED UP AND } \\
\text { RELEASED. }\end{array}$ & & & \\
\hline 8. & $\begin{array}{l}\text { All stored radioactive and mixed waste removed. } \\
\text { RADIOACTIVE AND MIXED WASTE WILL BE REMOVED. }\end{array}$ & & & \\
\hline 9. & $\begin{array}{l}\text { All hazardous materials used for deactivation and cleanup work must } \\
\text { be collected and disposed of in accordance with requirements. } \\
\text { ALL HAZARDOUS MATERIALS USED FOR DEACTIVATION AND CLEANUP WILL BE } \\
\text { REMOVED. }\end{array}$ & & & \\
\hline 10. & $\begin{array}{l}\text { A11 unattached hazardous materials (i.e., loose friable asbestos, } \\
\text { lead. mercury, etc.) must be removed from the facility and disposed } \\
\text { of in accordance with requirements. } \\
\text { ALL UNATTACHED HAZARDOUS WATERIALS WILL BE REMOVED. }\end{array}$ & & & \\
\hline 11. & $\begin{array}{l}\text { Attached hazardous materials located, identified, quantified, } \\
\text { labelled as necessary, and recorded. } \\
\text { ATTACEE HAZARDOUS MATERIALS WLL BE LOCATED, IDENTIFIED, } \\
\text { QUANTIFIED, LABELLED AS NECESSARY AND RECORDED. }\end{array}$ & & & \\
\hline 12. & $\begin{array}{l}\text { All reactor fuel elements and \or other source and special materials } \\
\text { must be removed from the building. }\end{array}$ & $\begin{array}{l}\text { NOT } \\
\text { APPLICABLE }\end{array}$ & & \\
\hline 13. & $\begin{array}{l}\text { Final radiological status surveys available. } \\
\text { FINAL RADIOLOGICAL SURVEYS WILL BE INCLUDED WITH THE DEACTIVATION } \\
\text { CHECKSHEET. }\end{array}$ & & & \\
\hline
\end{tabular}




\begin{tabular}{|c|c|c|c|c|}
\hline \multicolumn{5}{|c|}{ END POINT TABLE } \\
\hline \multirow[t]{2}{*}{ ITEM \# } & \multirow{2}{*}{ END POINT ACCEPTANCE CRITERIA - 334, 334A } & \multicolumn{2}{|c|}{ ACCEPTANCE INITIALS } & \multirow{2}{*}{$\begin{array}{l}\text { IMPLEMENTATION } \\
\text { VERIFICATION } \\
\text { DOCUMENT }\end{array}$} \\
\hline & & WHC & $\mathrm{BHI}$ & \\
\hline $\begin{array}{l}14 . \\
14 A\end{array}$ & $\begin{array}{l}\text { Tanks, vesse1s, drums, etc. drained and heels removed. } \\
\text { ALL TANKS, VESSELS, DRUMS, ETC. WILL BE DRAINED AND HEELS RENOVED. } \\
300 \text { WATS WILL BE CLEAN CLOSED. }\end{array}$ & & & \\
\hline 15. & $\begin{array}{l}\text { A11 elevator and crane systems are laid up with documentation of the } \\
\text { type, weight and class of fluid required for operation, should that } \\
\text { become desirable in the future. }\end{array}$ & $\begin{array}{l}\text { NOT } \\
\text { APPLICABLE }\end{array}$ & & \\
\hline 16. & $\begin{array}{l}\text { Documentation is available confirming that zero energy checks were } \\
\text { made on all de-energized electrical circuits and isolated } \\
\text { pressurized systems e.g., water, service air, etc. using existing } \\
\text { documents to the extent possible. }\end{array}$ & & & \\
\hline $16 A$ & $\begin{array}{l}\text { WATER, STEAM, AND SERVICE AIR WILL BE BLANKED AT LOCATION OUTSIDE } \\
\text { BUILDING. }\end{array}$ & & & \\
\hline $16 B$ & $\begin{array}{l}\text { IF ELECTRICAL SERVICE IS NOT DISCONNECTED BY CUTTING } \\
\text { THE FEED CABLES, ENERGY STATUS WILL BE DOCUMENTED ON ENERGIZED } \\
\text { ELECTRICAL SYSTEMS. }\end{array}$ & & & \\
\hline $\begin{array}{l}17 . \\
17 A .\end{array}$ & $\begin{array}{l}\text { Electrical systems reduced to that necessary for S\&M and subsequent } \\
\text { D\&D. This includes removal of emergency light fixtures and the } \\
\text { associated batteries. Where appropriate, centralize the remaining } \\
\text { electrical services to a single point. } \\
\text { ELECTRICAL SYSTEMS WILL BE APPROPRIATELY ISOLATED AND THE ISOLATION } \\
\text { DOCUMENTED, INCLUDING ISOLATION LOCATION(S). }\end{array}$ & & & \\
\hline 18. & $\begin{array}{l}\text { All heating, ventilation and air conditioning supply and exhaust air } \\
\text { systems shut down and de-energized. This criteria includes } \\
\text { evaluation and, as appropriate, the shutdown of the High-efficiency } \\
\text { particulate air-filtered (HEPA) ventilation exhaust from process } \\
\text { areas. }\end{array}$ & $\begin{array}{l}\text { NOT } \\
\text { APPLICABLE }\end{array}$ & & \\
\hline 19. & All unneeded equipment shut down. & $\begin{array}{l}\text { NOT } \\
\text { APPLICABLE }\end{array}$ & & \\
\hline
\end{tabular}




\begin{tabular}{|c|c|c|c|c|}
\hline \multicolumn{5}{|c|}{ END POINT TABLE } \\
\hline \multirow[t]{2}{*}{ ITEM \# } & \multirow[t]{2}{*}{ END POINT ACCEPTANCE CRITERIA - 334, 334A } & \multicolumn{2}{|c|}{ ACCEPTANCE INITIALS } & \multirow{2}{*}{$\begin{array}{l}\text { IMPLEMENTATION/ } \\
\text { VERIFICATION } \\
\text { DOCUMENT }\end{array}$} \\
\hline & & WHC & BHI & \\
\hline 20. & Fire protection systems downgraded. & & & \\
\hline 20A. & ALL PORTABLE FIRE EXTINGUISHERS WILL BE REMOVED FRON THE BUILDING. & & & \\
\hline 208. & THE FIRE ALARM SYSTEM WILL BE DEACTIVATED. & & & \\
\hline 21. & $\begin{array}{l}\text { The radiation space monitoring system should be reduced to a level } \\
\text { required by the Health Physics organization. }\end{array}$ & $\begin{array}{l}\text { NOT } \\
\text { APPLICABLE }\end{array}$ & & \\
\hline 22. & $\begin{array}{l}\text { The continuous air monitoring system should be reduced to a level } \\
\text { required by the Health Physics organization. }\end{array}$ & $\begin{array}{l}\text { NOT } \\
\text { APPLICABLE }\end{array}$ & & \\
\hline 23. & The criticality monitoring and alarm system should be deactivated. & $\begin{array}{l}\text { NOT } \\
\text { APPLICABLE }\end{array}$ & & \\
\hline 24. & $\begin{array}{l}\text { Reduce or el iminate the electrical, service air, and water supply } \\
\text { services to the building in accordance with the requirements for } \\
\text { S\&M. }\end{array}$ & & & \\
\hline $24 A$ & WATER SUPPLY WILL BE BLANKED AT LOCATION OUTSIDE THE BUILDING. & & & \\
\hline $24 \mathrm{~B}$. & ELECTRICAL SERVICE WILL BE REDUCED PER ITEM \#178. & & & \\
\hline 25. & $\begin{array}{l}\text { Remove all emergency lighting and dispose of associated batteries. } \\
\text { ALL EMERGENCY LIGHTING WILL BE REMOVED AND ASSOCIATED BATTERIES } \\
\text { DISPOSED. }\end{array}$ & & & \\
\hline 26. & The building steam system should be deactivated and tagged. & $\begin{array}{l}\text { NOT } \\
\text { APPLICABLE }\end{array}$ & & \\
\hline 27. & $\begin{array}{l}\text { All effluent flow routes to disposal sites are isolated by sealing } \\
\text { at the building. }\end{array}$ & & & \\
\hline
\end{tabular}

ज 
Rev. 0

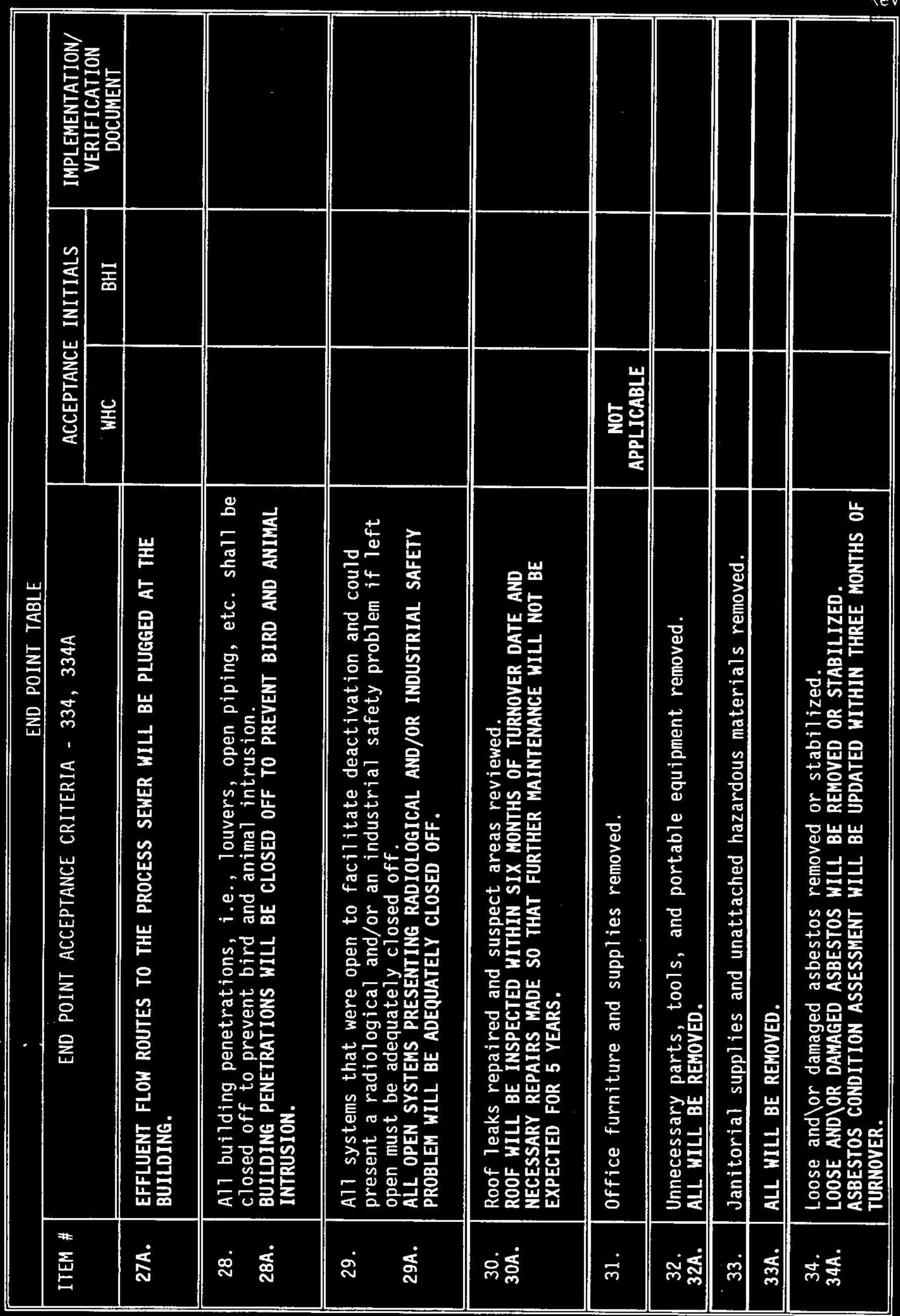




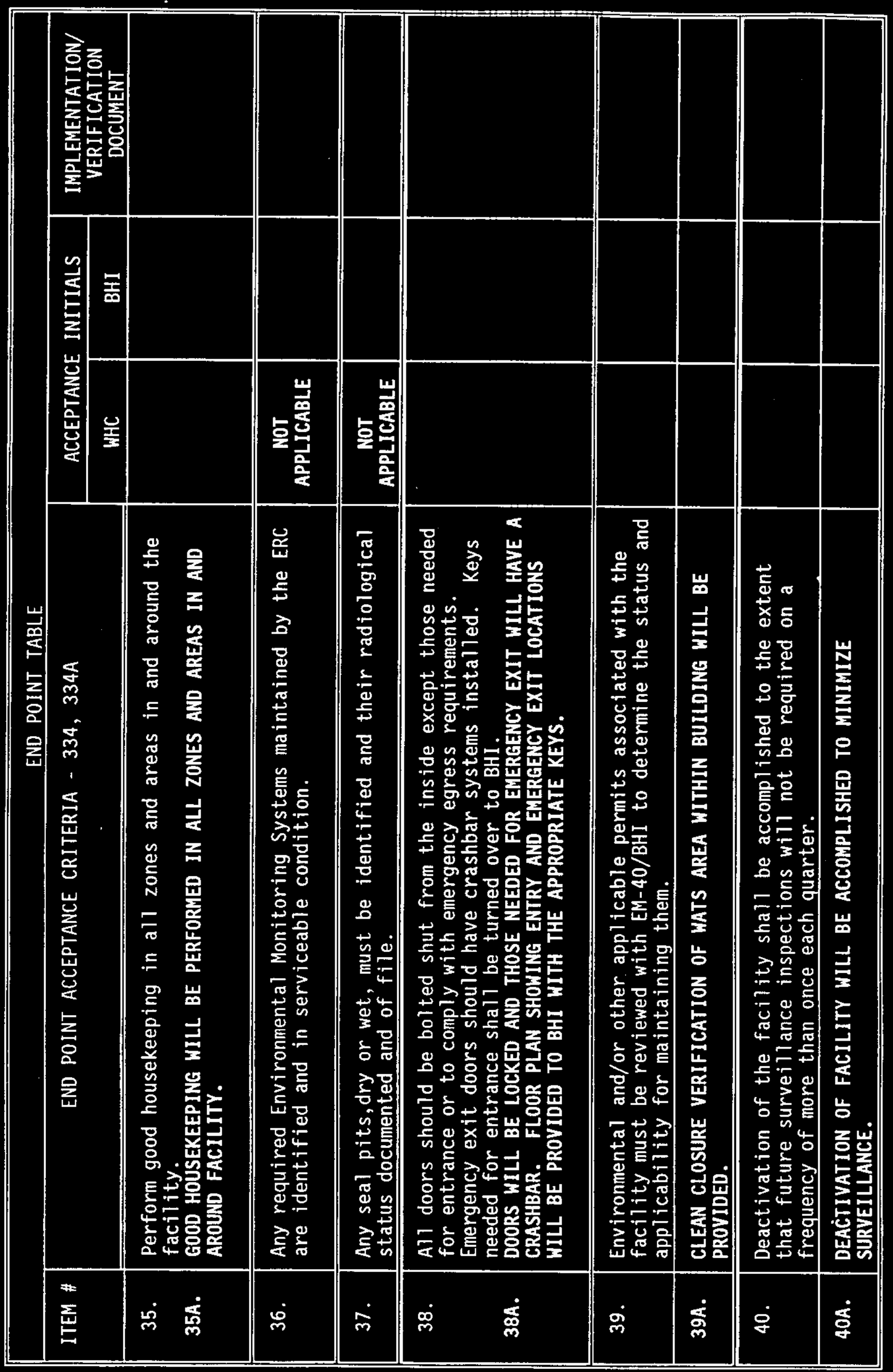




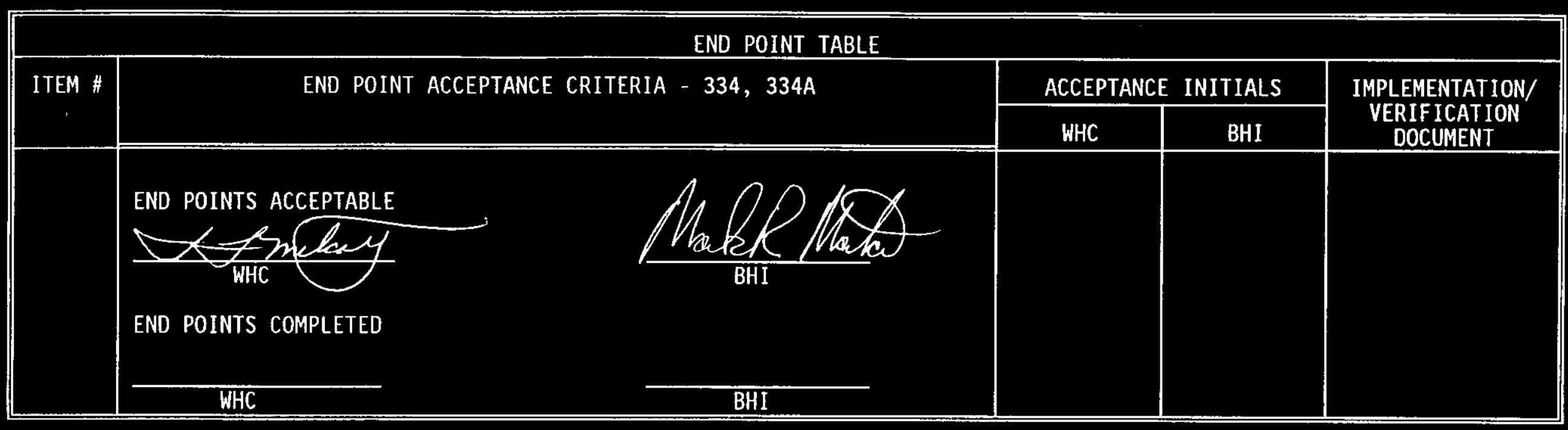




\begin{tabular}{|c|c|c|c|c|}
\hline \multicolumn{5}{|c|}{ END POINT TABLE } \\
\hline \multirow{2}{*}{ ITEM \# } & \multirow{2}{*}{ END POINT ACCEPTANCE CRITERIA - M0-052 TRAILER } & \multicolumn{2}{|c|}{ ACCEPTANCE INITIALS } & \multirow{2}{*}{$\begin{array}{l}\text { IMPLEMENTATION/ } \\
\text { VERIFICATION } \\
\text { DOCUMENT }\end{array}$} \\
\hline & & WHC & $\mathrm{BHI}$ & \\
\hline 1. & $\begin{array}{l}\text { Prepare and complete deact ivation checksheet. } \\
\text { CHECKSHEET WILL BE PREPARED, COMPLETED AND SIGNED OFF BY BOTH } \\
\text { ORGANIZATIONS (WHC AND BHI). }\end{array}$ & & & \\
\hline 2. & $\begin{array}{l}\text { A11 pending Radiation Occurrence reports, Unusual Occurrence Reports } \\
\text { and } \backslash \text { or any other out-of-standard condition reports finalized and } \\
\text { closed out in accordance with applicable MRPs. No operable } \\
\text { emergency sirens shall remain mounted on or powered from the } \\
\text { building. }\end{array}$ & & & \\
\hline $2 A$. & $\begin{array}{l}\text { RADIATION OCCURRENCE REPORTS, UNUSUAL OCCURRENCE REPORTS ANDJOR ANY } \\
\text { OTHER OUT-OF-STANDARD CONDITIONS REPORTS WILL BE FINALIZED AND } \\
\text { CLOSED BY WHC. }\end{array}$ & & & \\
\hline 3. & $\begin{array}{l}\text { The Fuel Supply Shutdown Facilities Interim Safety Basis Report and } \\
\text { Plant Emergency Procedures shall be reviewed and updated for } \\
\text { deactivation/shutdown status. }\end{array}$ & & & \\
\hline $3 A$. & $\begin{array}{l}\text { REVIEN AND UPDATE OF THE INTERIM SAFETY BASIS REPORT WILL BE } \\
\text { COMPLETED AND PROVIDED TO BHI } 6 \text { MONTHS PRIOR TO TURNOVER. } \\
\end{array}$ & & & \\
\hline 3B. & $\begin{array}{l}\text { REVIEW AND UPDATE OF THE PLANT EMERGENCY PROCEDURES WILL BE } \\
\text { COMPLETED AND PROVIDED TO BHI } 6 \text { MONTHS PRIOR TO TURNOVER. }\end{array}$ & & & \\
\hline 4 . & $\begin{array}{l}\text { CVI index, drawings, procedures, prints, photographs, history, } \\
\text { design and operat ing documents provided. } \\
\text { AVAILABLE ESSENTIAL DRAWINGS, A DRAWING LIST, PROCEDURES, ETC. WILL } \\
\text { BE PROVIDED IN TURNOVER FILE. }\end{array}$ & & & \\
\hline 5. & $\begin{array}{l}\text { All permanent radiologically contaminated areas to be decontaminated } \\
\text { and released or the surface contamination levels reduced or fixed in } \\
\text { place to minimize re-suspension and } l o r \text { migration of loose } \\
\text { contamination. }\end{array}$ & $\begin{array}{l}\text { NOT } \\
\text { APPLICABLE }\end{array}$ & & \\
\hline 6. & $\begin{array}{l}\text { Ful1 compliance with Hanford Site Radiological Control Manual must } \\
\text { be assured, especially as it pertains to radiological post ing. } \\
\text { COMPLIANCE WITH HANFORD SITE RADIOLOGICAL CONTROL MANUAL WILL BE } \\
\text { CONFIRMED BY HAVING } 300 \text { AREA RADIOLOGICAL CONTROL PERFORM AN } \\
\text { ASSESSHENT AND WHC WILL CORRECT ALL NOTED DISCREPANCIES. }\end{array}$ & & & \\
\hline
\end{tabular}




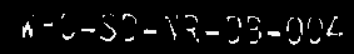

Rev. 0

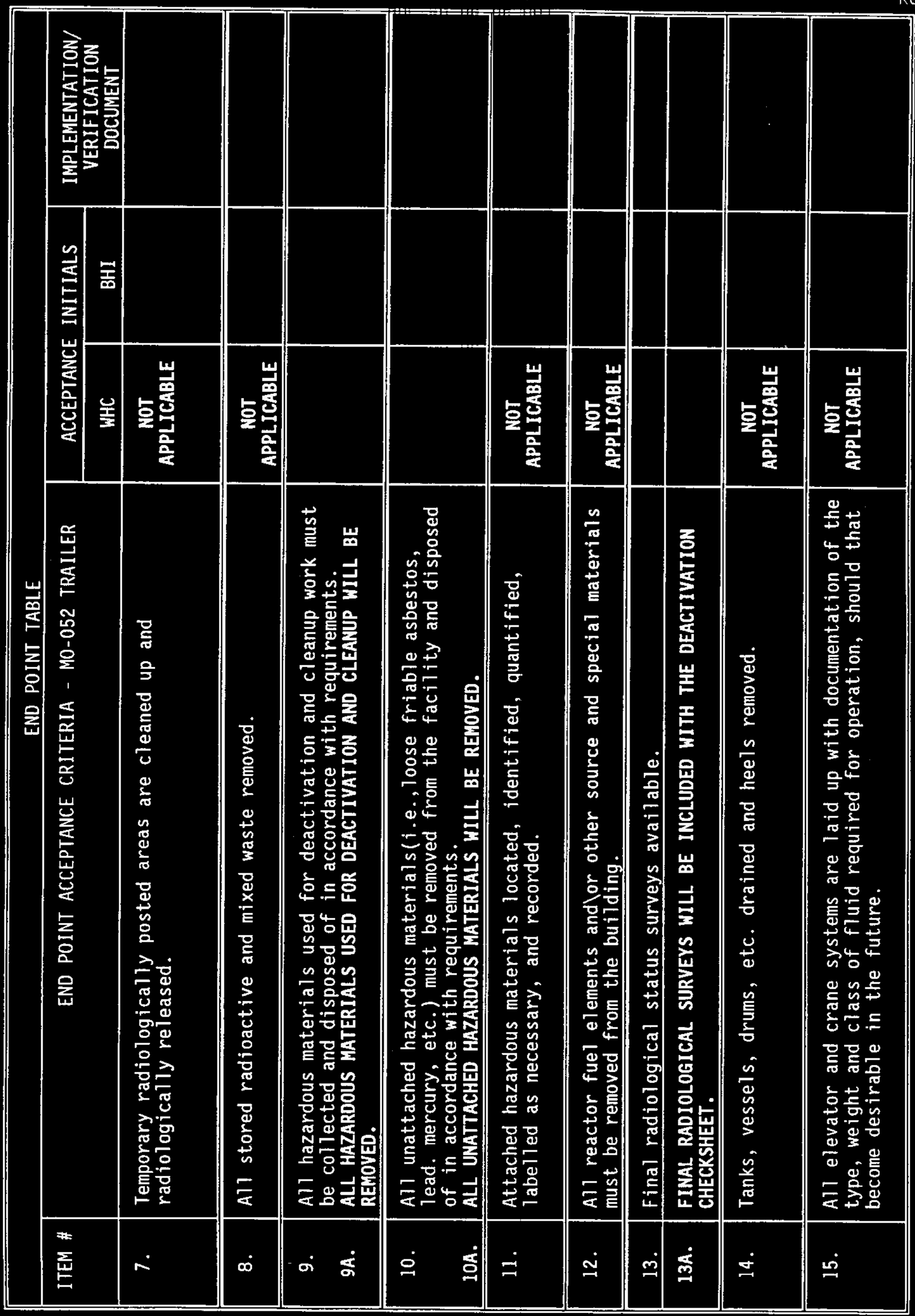


$n-c-2 j-18-3,3-10<2$

Rev. 0

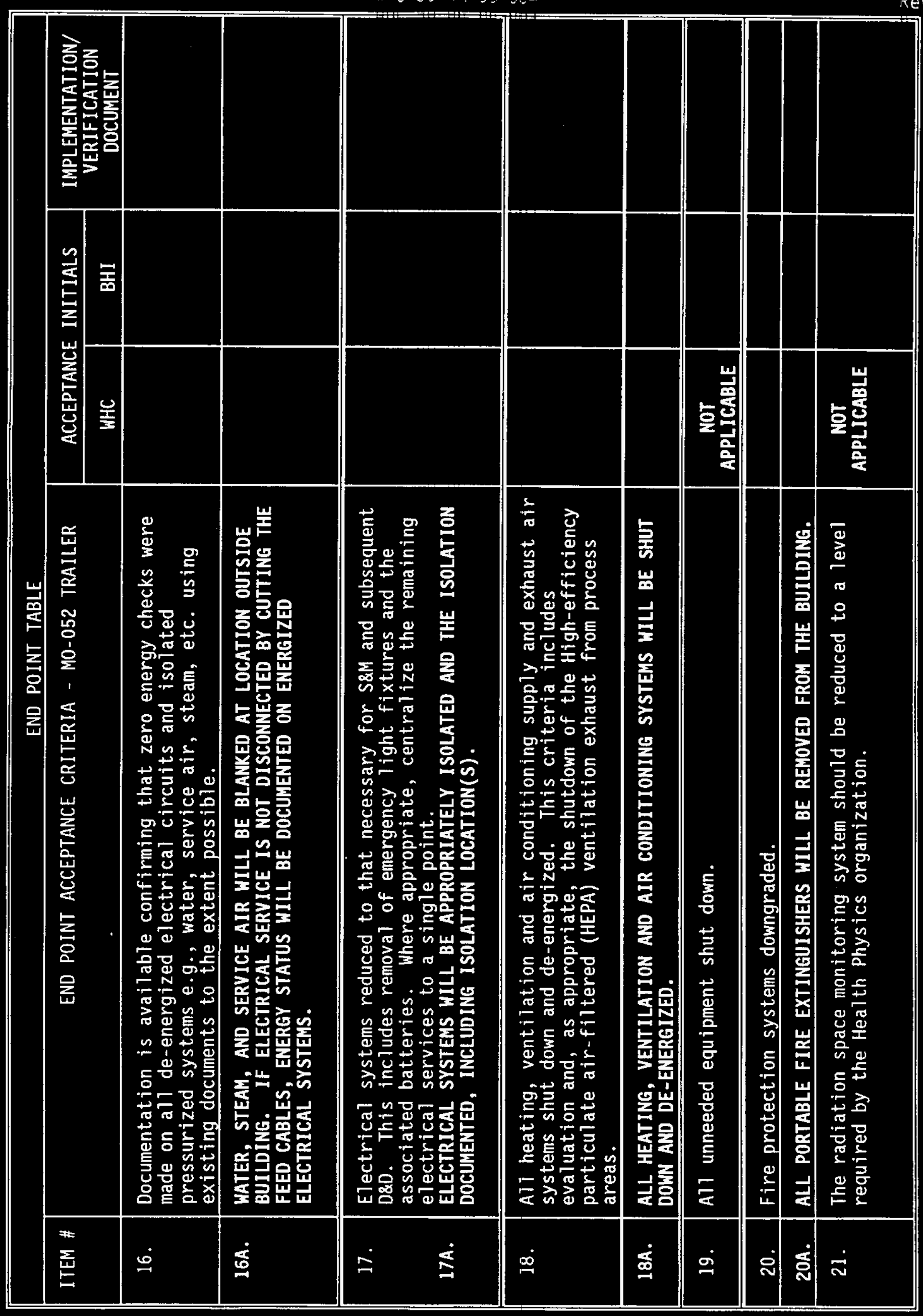




\begin{tabular}{|c|c|c|c|c|}
\hline \multicolumn{5}{|c|}{ END POINT TABLE } \\
\hline \multirow[t]{2}{*}{ ITEM \# } & \multirow[t]{2}{*}{ END POINT ACCEPTANCE CRITERIA - M0-052 TRAILER } & \multicolumn{2}{|c|}{ ACCEPTANCE INITIALS } & \multirow{2}{*}{$\begin{array}{l}\text { IMPLEMENTATION/ } \\
\text { VERIFICATION } \\
\text { DOCUMENT }\end{array}$} \\
\hline & & WHC & BHI & \\
\hline 22. & $\begin{array}{l}\text { The continuous air monitoring system should be reduced to a level } \\
\text { required by the Health Physics organization. }\end{array}$ & $\begin{array}{l}\text { NOT } \\
\text { APPLICABLE }\end{array}$ & & \\
\hline 23. & The criticality monitoring and alarm system should be deactivated. & $\begin{array}{l}\text { NOT } \\
\text { APPLICABLE } \\
\end{array}$ & & \\
\hline 24. & $\begin{array}{l}\text { Reduce or eliminate the electrical and water supply services to the } \\
\text { building in accordance with the requirements for S\&M. }\end{array}$ & $\cdot$ & & \\
\hline $24 A$. & WATER SUPPLY WILL BE BLANKED AT LOCATION OUTSIDE THE BUILDING. & & & \\
\hline 24B. & ELECTRICAL SERVICE WILL BE REDUCED PER ITEM \#17. & & & \\
\hline 25. & Remove all emergency lighting and dispose of associated batteries. & & & \\
\hline 25A. & $\begin{array}{l}\text { ALL EMERGENCY LIGHTING WILL BE REMOVED AWD ASSOCIATED BATTERIES } \\
\text { OISPOSED. }\end{array}$ & & & \\
\hline 26. & The building steam system should be deactivated and tagged. & $\begin{array}{c}\text { NOT } \\
\text { APPLICABLE } \\
\end{array}$ & & \\
\hline 27. & $\begin{array}{l}\text { All effluent flow routes to disposal sites are isolated by sealing } \\
\text { at the building. }\end{array}$ & & & \\
\hline $27 \mathrm{~A}$. & $\begin{array}{l}\text { SANITARY SEWER FLOW ROUTE WILL BE ISOLATED BY SEALING AT THE } \\
\text { BUILDING. }\end{array}$ & & & \\
\hline 28. & $\begin{array}{l}\text { All building penetrations, i.e., louvers, open piping, etc. shall be } \\
\text { closed off to prevent bird and animal intrusion. }\end{array}$ & & & \\
\hline 28A. & $\begin{array}{l}\text { BUILDING PENETRATIONS WILL BE CLOSED OFF TO PREVENT BIRD AND ANIMAL } \\
\text { INTRUSION. }\end{array}$ & & & \\
\hline 29A. & $\begin{array}{l}\text { All systems that were open to facilitate deactivation and could } \\
\text { present a radiological and/or an industrial safety problem if left } \\
\text { open must be adequately closed off. } \\
\text { ALL OPEN SYSTEMS PRESENTING RADIOLOGICAL AND/OR INDUSTRIAL SAFETY } \\
\text { PROBLEM WILL BE ADEQUATELY CLOSED OFF. }\end{array}$ & & & \\
\hline
\end{tabular}




\begin{tabular}{|c|c|c|c|c|}
\hline \multicolumn{5}{|c|}{ END POINT TABLE } \\
\hline \multirow[t]{2}{*}{ ITEM \# } & \multirow[t]{2}{*}{ END POINT ACCEPTANCE CRITERIA - M0-052 TRAILER } & \multicolumn{2}{|c|}{ ACCEPTANCE INITIALS } & \multirow{2}{*}{$\begin{array}{l}\text { IMPLEMENTATION/ } \\
\text { VERIF ICATION } \\
\text { DOCUMENT }\end{array}$} \\
\hline & & WHC & BHI & \\
\hline 30. & Roof leaks repaired and suspect areas reviewed. & & & \\
\hline $30 A$. & $\begin{array}{l}\text { ROOF WILL BE INSPECTED WITHIN SIX MONTHS OF TURNOVER DATE AND } \\
\text { NECESSARY REPAIRS MADE SO THAT FURTHER MAINTENANCE WILL NOT BE } \\
\text { EXPECTED FOR } 5 \text { YEARS. }\end{array}$ & & & \\
\hline 31. & Office furniture and supplies removed. & & & \\
\hline $31 \mathrm{~A}$. & OFFICE FURNITURE AND SUPPLIES WILL BE REMOVED. & & & \\
\hline 32. & Unnecessary parts, tools, and portable equipment removed. & $\begin{array}{l}\text { NOT } \\
\text { APPLICABLE }\end{array}$ & & \\
\hline 33. & Janitorial supplies and unattached hazardous materials removed. & & & \\
\hline 33A. & $\begin{array}{l}\text { JANITORIAL SUPPLIES AND UNATTACHED HAZARDOUS MATERIALS WILL BE } \\
\text { REMOVED. }\end{array}$ & & & \\
\hline 34. & Loose and \or damaged asbestos removed or stabilized. & & & \\
\hline $34 A$. & $\begin{array}{l}\text { LOOSE AND YOR DAMAGED ASBESTOS WILL BE REMOVED OR STABILIZED. ASBESTOS } \\
\text { CONDITION ASSESSMENT WILL BE UPDATED WITHIN THREE MONTHS OF } \\
\text { TURNOVER. }\end{array}$ & & & \\
\hline 35. & $\begin{array}{l}\text { Perform good housekeeping in all zones and areas in and around the } \\
\text { facility. }\end{array}$ & & & \\
\hline $35 A$. & $\begin{array}{l}\text { GOOD HOUSEKEEPING WILL BE PERFORMED IN ALL ZONES AND AREAS IN AND } \\
\text { AROUND FACILITY. }\end{array}$ & & & \\
\hline 36. & $\begin{array}{l}\text { Any required Environmental Monitoring Systems maintained by the ERC } \\
\text { are identified and in serviceable condition. }\end{array}$ & $\begin{array}{l}\text { NOT } \\
\text { APPLICABLE }\end{array}$ & & \\
\hline 37. & $\begin{array}{l}\text { Any seal pits, dry or wet, must be identified and their radiological } \\
\text { status documented and of file. }\end{array}$ & $\begin{array}{l}\text { NOT } \\
\text { APPLICABLE }\end{array}$ & & \\
\hline
\end{tabular}

g 
$n-5-5-12-73-006$

Rev. 0

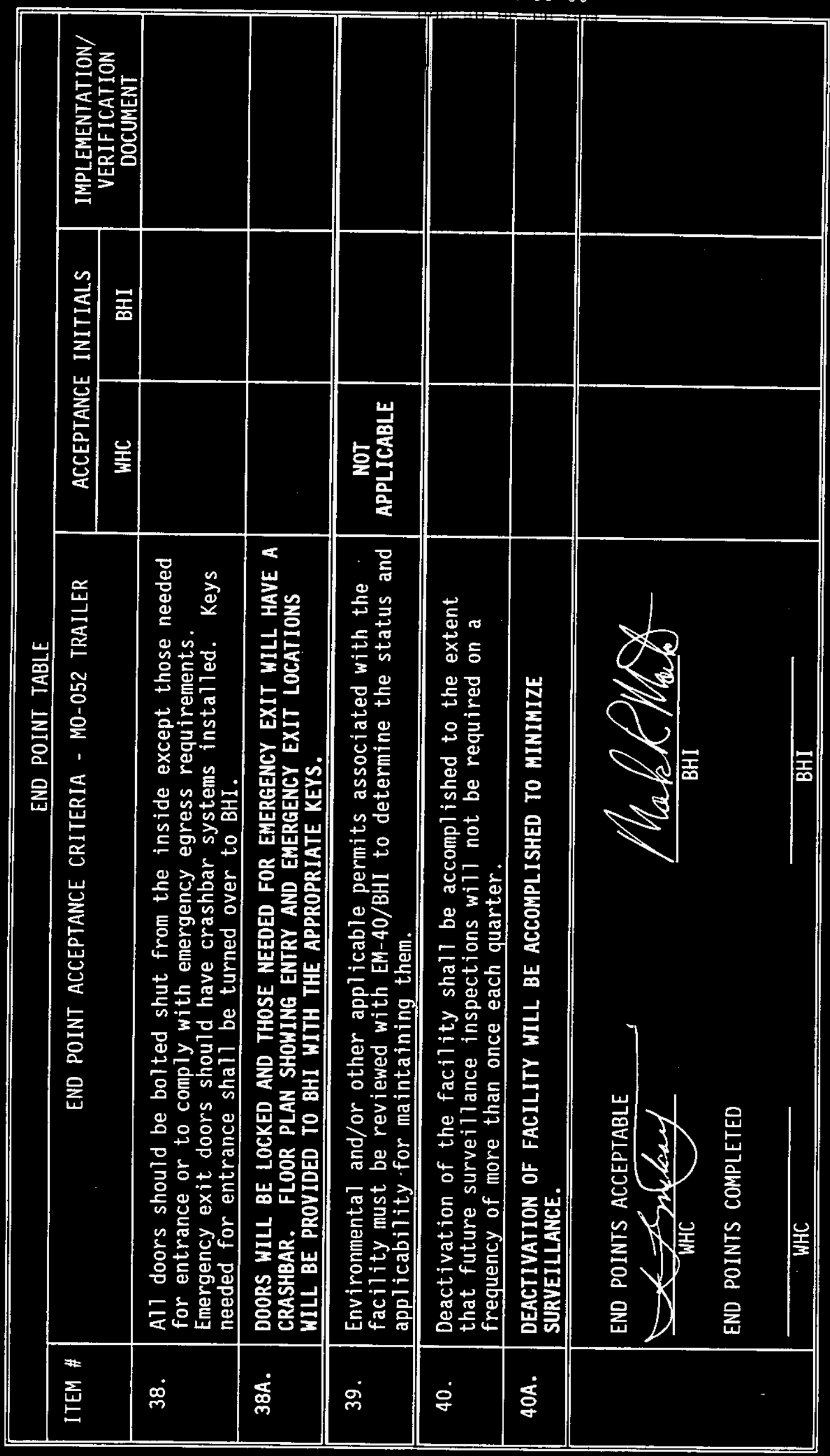




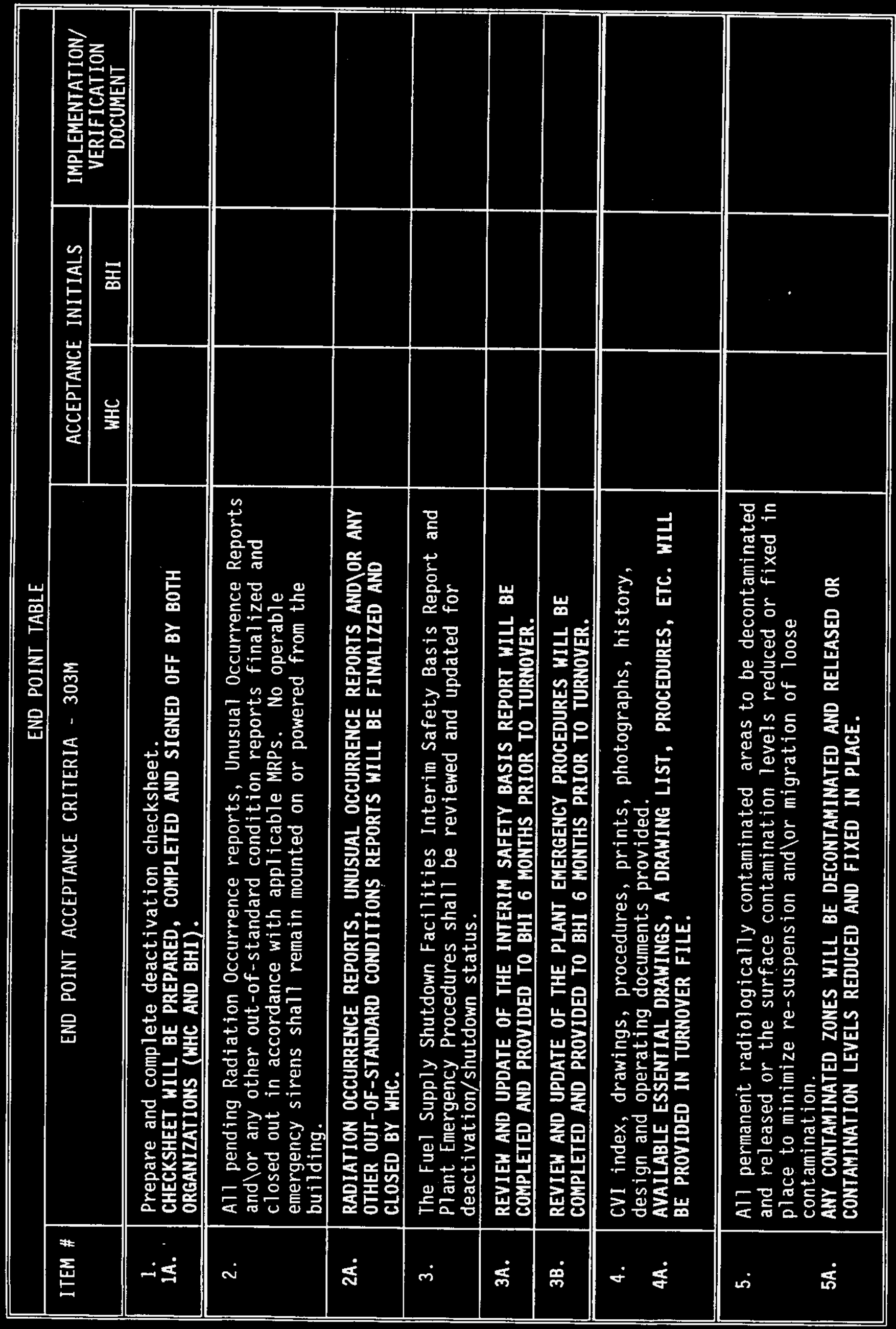




\begin{tabular}{|c|c|c|c|c|}
\hline \multicolumn{5}{|c|}{ END POINT TABLE } \\
\hline \multirow[t]{2}{*}{ ITEM \# } & \multirow[t]{2}{*}{ END POINT ACCEPTANCE CRITERIA - 303M } & \multicolumn{2}{|c|}{ ACCEPTANCE INITIALS } & \multirow{2}{*}{$\begin{array}{l}\text { IMPLEMENTATION } \\
\text { VERIFICATION } \\
\text { DOCUMENT }\end{array}$} \\
\hline & & WHC & BHI & \\
\hline 6 . & $\begin{array}{l}\text { Ful1 compliance with Hanford Site Radiological Control Manual must } \\
\text { be assured, especially as it pertains to radiological posting. } \\
\text { CONPLIANCE WITH HANFORD SITE RADIOLOGICAL CONTROL MANUAL WILL BE } \\
\text { CONFIRMED BY HAVING 300 AREA RADIOLOGICAL CONTROL PERFORM AN } \\
\text { ASSESSMENT AND WHC WILL CORRECT ALL NOTED DISCREPANCIES. }\end{array}$ & & & \\
\hline 7 . & $\begin{array}{l}\text { Temporary radiologically posted areas are cleaned up and } \\
\text { radiologically released. } \\
\text { TEMPORARY RADIOLOGICALLY POSTED AREAS WILL BE CLEANED UP AND } \\
\text { RELEASED. }\end{array}$ & & & \\
\hline 8. & A11 stored radioactive and mixed waste removed. & & & \\
\hline $8 A$. & RADIOACTIVE AND MIXED WASTE WILL BE REMOVED. & & & \\
\hline $8 B$. & HEPA FILTER WILL BE REMOVED. & & & \\
\hline 8C. & DUST COLLECTOR BAGS WILL BE REMOVED. & & & \\
\hline 9. & $\begin{array}{l}\text { A11 hazardous materials used for deactivation and cleanup work must } \\
\text { be collected and disposed of in accordance with requirements. } \\
\text { ALL HAZARDOUS MATERIALS USED FOR DEACTIVATION AND CLEANUP WILL BE } \\
\text { REMOVED. }\end{array}$ & & & \\
\hline 10. & $\begin{array}{l}\text { All unattached hazardous materials (i.e., loose friable asbestos, } \\
\text { lead. mercury, etc.) must be removed from the facility and disposed } \\
\text { of in accordance with requirements. } \\
\text { ALL UNATTACHED HAZARDOUS MATERIALS WILL BE REMOVED. }\end{array}$ & & & \\
\hline 11. & $\begin{array}{l}\text { Attached hazardous materiais located, identified, quantified, } \\
\text { labelled as necessary, and recorded. } \\
\text { ATTACHED HAZARDOUS MATERIALS WILL BE LOCATED, IDENT IFIED, } \\
\text { QUANTIFIED, LABELLED AS NECESSARY AND RECORDED. }\end{array}$ & & & \\
\hline 12. & $\begin{array}{l}\text { A11 reactor fuel elements and lor other source and special materials } \\
\text { must be removed from the building. } \\
\text { ALL SPECIAL NUCLEAR MATERIAL WILL BE REMOVED FROM THE BUILDING. }\end{array}$ & & & \\
\hline
\end{tabular}




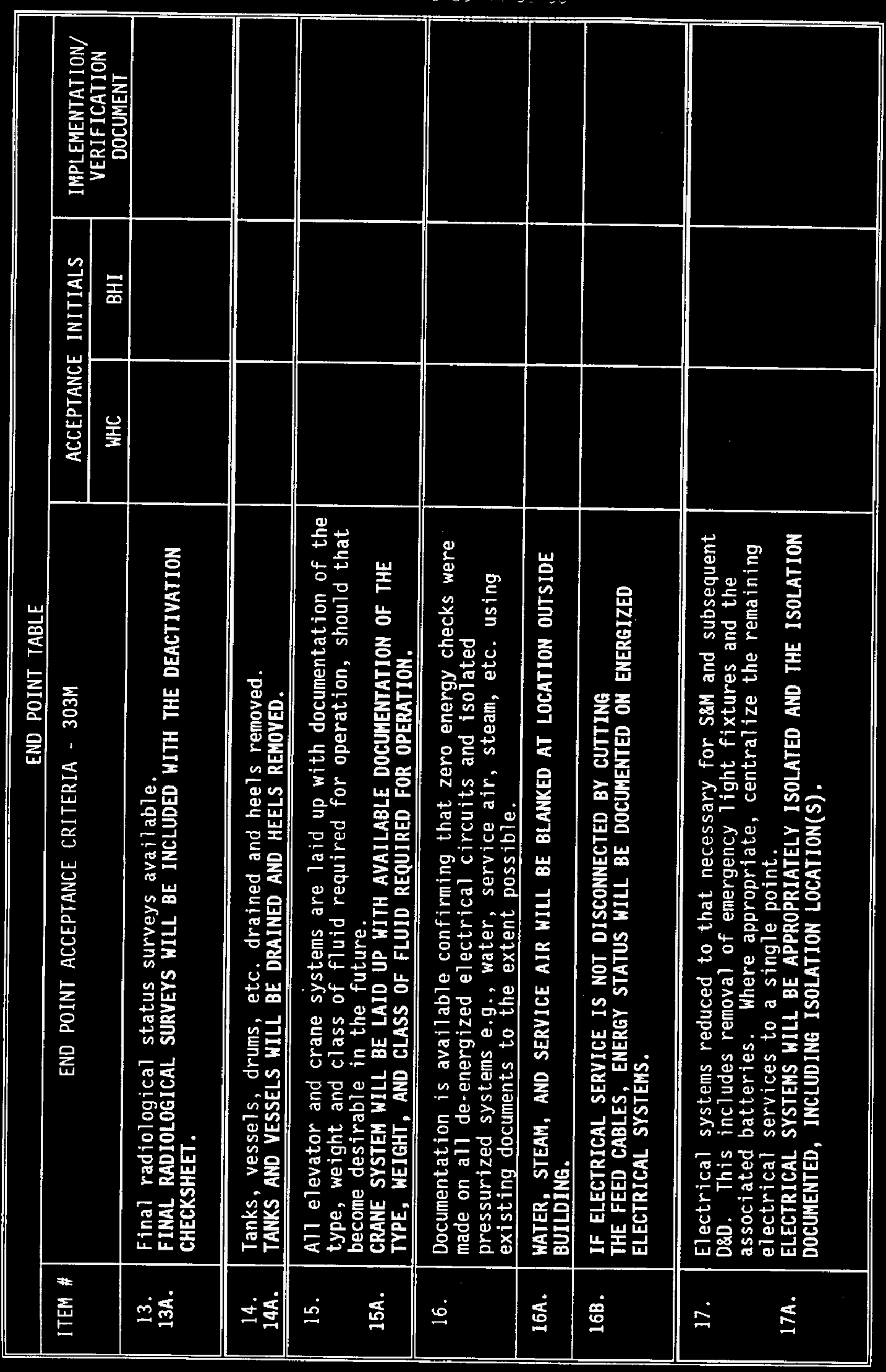

Rev. 0 
REV.

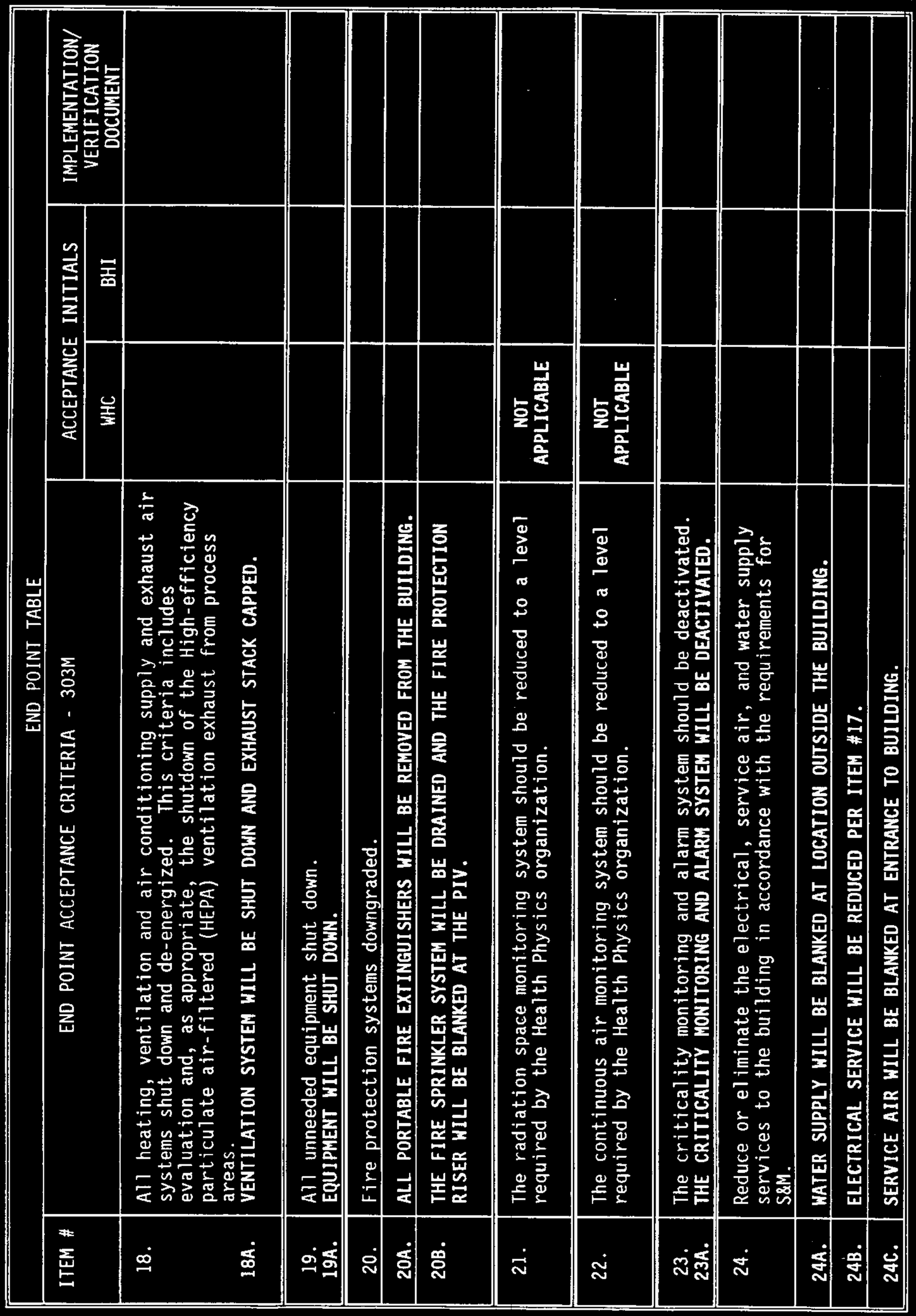




\begin{tabular}{|c|c|c|c|c|}
\hline \multicolumn{5}{|c|}{ END POINT TABLE } \\
\hline \multirow[t]{2}{*}{ ITEM \# } & \multirow[t]{2}{*}{ END POINT ACCEPTANCE CRITERIA - 303M } & \multicolumn{2}{|c|}{ ACCEPTANCE INITIALS } & \multirow{2}{*}{$\begin{array}{l}\text { IMPLEMENTATION/ } \\
\text { VERIF ICATION } \\
\text { DOCUMENT }\end{array}$} \\
\hline & & WHC & BHI & \\
\hline 25 & $\begin{array}{l}\text { Remove all emergency lighting and dispose of associated batteries. } \\
\text { ALL EMERGENCY LIGHTING WILL BE REMOVED AND ASSOCIATED BATTERIES } \\
\text { DISPOSED. }\end{array}$ & & & \\
\hline 26. & The building steam system should be deactivated and tagged. & $\begin{array}{l}\text { NOT } \\
\text { APPLICABLE }\end{array}$ & & \\
\hline 27 . & $\begin{array}{l}\text { All effluent flow routes to disposal sites are isolated by sealing } \\
\text { at the building. }\end{array}$ & & & \\
\hline $27 A$ & $\begin{array}{l}\text { EFFLUENT FLOW ROUTES TO THE PROCESS SEWER WILL BE PLUGGED AT THE } \\
\text { BUILDING. }\end{array}$ & & & \\
\hline $27 \mathrm{~B}$. & EFFLUENT FLOW ROUTES TO THE SANITARY SEWER UILL BE PLUGGED. & & & \\
\hline 28. & $\begin{array}{l}\text { All building penetrations, i.e., louvers, open piping, etc. shall be } \\
\text { closed off to prevent bird and animal intrusion. } \\
\text { BUILDING PENETRATIONS WILL BE CLOSED OFF TO PREVENT BIRD AND ANIMAL } \\
\text { INTRUSION. }\end{array}$ & & & \\
\hline $\begin{array}{l}29 . \\
29 A .\end{array}$ & $\begin{array}{l}\text { All systems that were open to facilitate deactivation and could } \\
\text { present a radiological and/or an industrial safety problem if left } \\
\text { open must be adequately closed off. } \\
\text { ALL OPEN SYSTEMS PRESENTING RADIOLOGICAL AND/OR INDUSTRIAL SAFETY } \\
\text { PROBLEM WILL BE ADEQUATELY CLOSED OFF. }\end{array}$ & & & \\
\hline 30 & $\begin{array}{l}\text { Roof leaks repaired and suspect areas reviewed. } \\
\text { ROOF WILL BE INSPECTED WITHIN SIX MONTHS OF TURNOVER DATE AND } \\
\text { NECESSARY REPAIRS MADE SO THAT FURTHER MAINTENANCE WILL NOT BE } \\
\text { EXPECTED FOR } 5 \text { YEARS. }\end{array}$ & & & \\
\hline 31. & Office furniture and supplies removed. & $\begin{array}{l}\text { NOT } \\
\text { APPLICABLE }\end{array}$ & & \\
\hline $\begin{array}{l}32 \text {. } \\
32 \mathrm{~A} \text {. }\end{array}$ & $\begin{array}{l}\text { Unnecessary parts, tools, and portable equipment removed. } \\
\text { ALL WILL BE REMOVED. }\end{array}$ & & & \\
\hline
\end{tabular}


$n^{-}-3-3-1+-2=-20$

Rev.

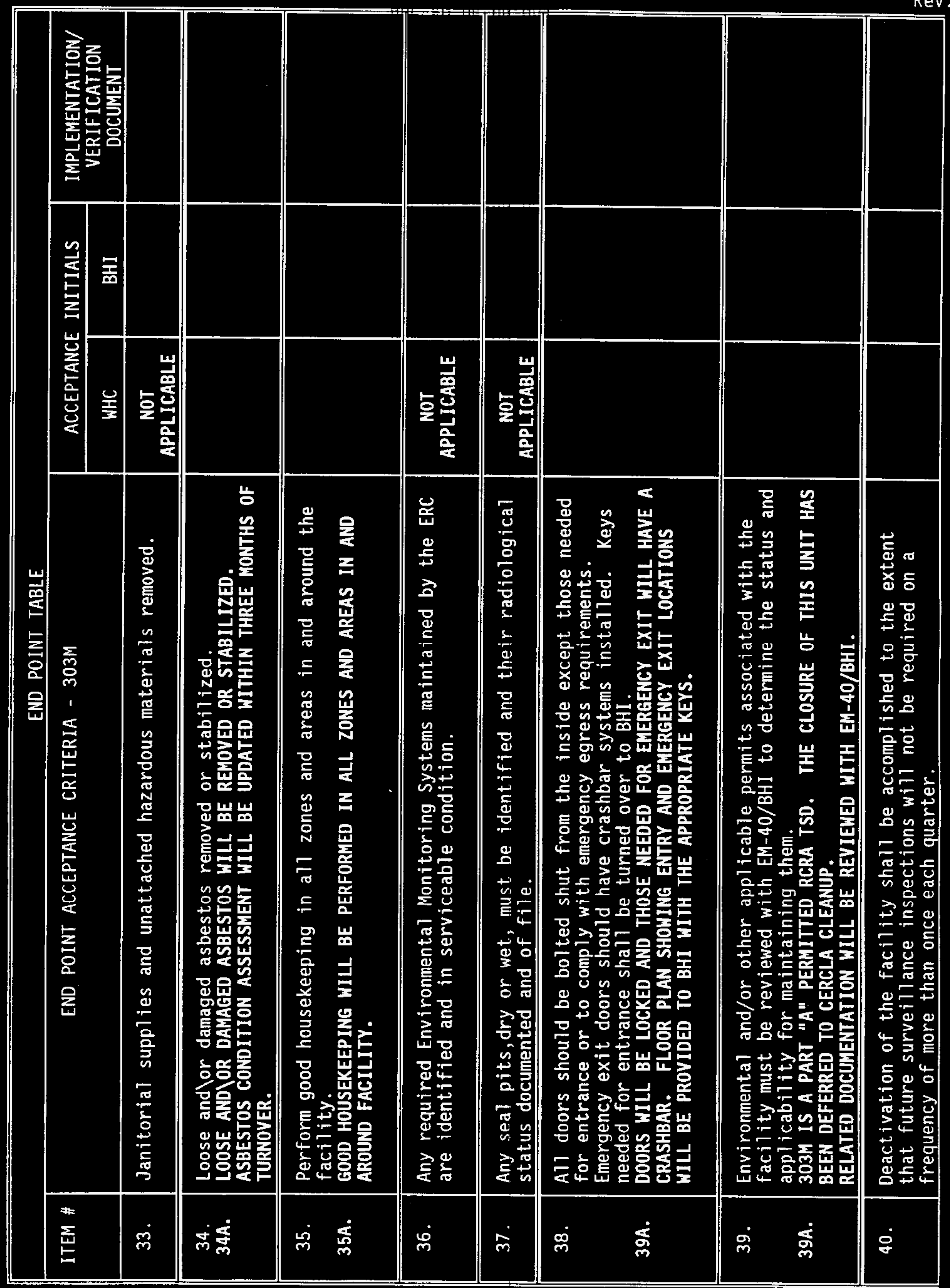




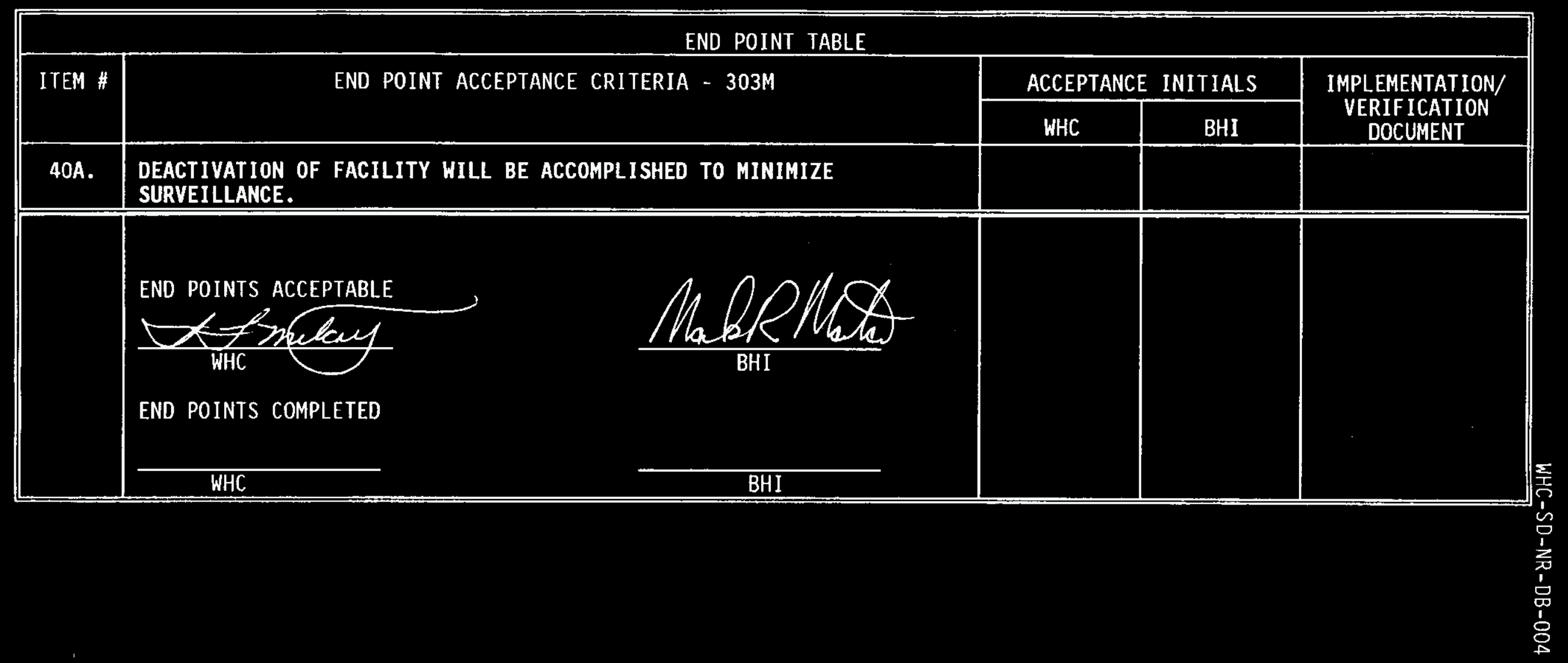




\begin{tabular}{|c|c|c|c|c|}
\hline \multicolumn{5}{|c|}{ END POINT TABLE } \\
\hline \multirow[t]{2}{*}{ ITEM \# } & \multirow[t]{2}{*}{ END POINT ACCEPTANCE CRITERIA - 303K, 3707G } & \multicolumn{2}{|c|}{ ACCEPTANCE INITIALS } & \multirow{2}{*}{$\begin{array}{l}\text { IMPLEMENTATION/ } \\
\text { VERIFICATION } \\
\text { DOCUMENT }\end{array}$} \\
\hline & & WHC & BHI & \\
\hline $\begin{array}{l}1 . \\
1 \mathrm{~A} .\end{array}$ & $\begin{array}{l}\text { Prepare and complete deactivation checksheet. } \\
\text { CHECKSHEET WILL BE PREPARED, COMPLETED AND SIGNED OFF BY BOTH } \\
\text { ORGANIZATIONS (WHC AND BHI). }\end{array}$ & & & \\
\hline 2. & $\begin{array}{l}\text { Al1 pending Radiation Occurrence reports, Unusual Occurrence Reports } \\
\text { and } \backslash \text { or any other out-of-standard condition reports finalized and } \\
\text { closed out in accordance with applicable MRPs. No operable } \\
\text { emergency sirens shall remain mounted on or powered from the } \\
\text { building. }\end{array}$ & & & \\
\hline $2 A$. & $\begin{array}{l}\text { RADIATION OCCURRENCE REPORTS, UNUSUAL OCCURRENCE REPORTS AND OR ANY } \\
\text { OTHER OUT-OF-STANDARO CONDITIONS REPORTS WILL BE FINALIZED AND } \\
\text { CLOSED BY WHC. }\end{array}$ & & & \\
\hline 3. & $\begin{array}{l}\text { The Fuel Supply Shutdown Facilities Interim Safety Basis Report and } \\
\text { Plant Emergency Procedures shall be reviewed and updated for } \\
\text { deactivation/shutdown status. }\end{array}$ & & & \\
\hline 3A. & $\begin{array}{l}\text { REVIEW AND UPDATE OF THE INTERIM SAFETY BASIS REPORT WILL BE } \\
\text { COMPLETED AND PROVIDED TO BHI } 6 \text { MONTHS PRIOR TO TURNOVER. }\end{array}$ & & & \\
\hline 3B. & $\begin{array}{l}\text { REVIEN AND UPDATE OF THE PLANT ENERGENCY PROCEDURES WILL BE } \\
\text { COMPLETED AND PROVIDED TO BHI } 6 \text { NONTHS PRIOR TO TURNOVER. }\end{array}$ & & & \\
\hline $\begin{array}{l}4 . \\
4 A .\end{array}$ & $\begin{array}{l}\text { CVI index, drawings, procedures, prints, photographs, history, } \\
\text { design and operating documents provided. } \\
\text { AVAILABLE ESSENTIAL DRAWINGS, A DRAWING LIST, PROCEDURES, ETC. WILL } \\
\text { BE PROVIDED IN TURNOVER FILE. }\end{array}$ & & & \\
\hline 5A. & $\begin{array}{l}\text { A11 permanent radiologically contaminated areas to be decontaminated } \\
\text { and released or the surface contamination levels reduced or fixed in } \\
\text { place to minimize re-suspension and } \backslash \text { or migration of loose } \\
\text { contamination. } \\
\text { CONTANINATED ZONES WILL BE DECONTAMINATED AND RELEASED OR } \\
\text { CONTAMINATION LEVELS REDUCED AND FIXED IN PLACE. }\end{array}$ & & & \\
\hline
\end{tabular}


$\because$

\begin{tabular}{|c|c|c|c|c|}
\hline \multicolumn{5}{|c|}{ END POINT TABLE } \\
\hline \multirow[t]{2}{*}{ ITEM \# } & \multirow[t]{2}{*}{ END POINT ACCEPTANCE CRITERIA - 303K, 3707G } & \multicolumn{2}{|c|}{ ACCEPTANCE INITIALS } & \multirow{2}{*}{$\begin{array}{l}\text { IMPLEMENTATION/ } \\
\text { VERIF ICATION } \\
\text { DOCUMENT }\end{array}$} \\
\hline & & WHC & BHI & \\
\hline 6. & $\begin{array}{l}\text { Full compliance with Hanford Site Radiological Control Manual must } \\
\text { be assured, especially as it pertains to radiological post ing. } \\
\text { COMPLIANCE WITH HANFORD SITE RADIOLOGICAL CONTROL MANUAL WILL BE } \\
\text { CONFIRMED BY HAVING } 300 \text { AREA RADIOLOGICAL CONTROL PERFORH AN } \\
\text { ASSESSMENT AND WHC WILL CORRECT ALL NOTED DISCREPANCIES. }\end{array}$ & & & \\
\hline 7. & $\begin{array}{l}\text { Temporary radiologically posted areas are cleaned up and } \\
\text { radiologically released. } \\
\text { TEMPORARY RADIOLOGICALLY POSTED AREAS WILL BE CLEANED UP AND } \\
\text { RELEASED. }\end{array}$ & & & \\
\hline 8. & $\begin{array}{l}\text { All stored radioactive and mixed waste removed. } \\
\text { RADIOACTIVE AND MIXED WASTE WILL BE REMOVED. }\end{array}$ & & & \\
\hline 9. & $\begin{array}{l}\text { A1l hazardous materials used for deactivation and cleanup work must } \\
\text { be collected and disposed of in accordance with requirements. } \\
\text { ALL HAZARDOUS MATERIALS USED FOR DEACTIVATION AND CLEANUP WILL BE } \\
\text { REMOVED. }\end{array}$ & & & \\
\hline 10. & $\begin{array}{l}\text { All unattached hazardous materials (i.e., loose friable asbestos, } \\
\text { lead. mercury, etc.) must be removed from the facility and disposed } \\
\text { of in accordance with requirements. } \\
\text { ALL UNATTACHED HAZARDOUS MATERIALS WILL BE REMOVED. }\end{array}$ & & & \\
\hline 11. & $\begin{array}{l}\text { Attached hazardous materials located, identified, quantified, } \\
\text { labelled as necessary, and recorded. } \\
\text { ATTACHED HAZARDOUS MATERIALS WILL BE LOCATED, IDENTIFIED, } \\
\text { QUANT IFIED, LABELLED AS NECESSARY AND RECORDED. }\end{array}$ & & & \\
\hline 12. & $\begin{array}{l}\text { All reactor fuel elements and } \backslash \text { or other source and special materials } \\
\text { must be removed from the building. } \\
\text { ALL SPECIAL NUCLEAR MATERIAL WILL BE REHOVED FROM THE BUILDING. }\end{array}$ & & & \\
\hline 13 & $\begin{array}{l}\text { Final radiological status surveys available. } \\
\text { FINAL RADIOLOGICAL SURVEYS WILL BE INCLUDED WITH THE DEACTIVATION } \\
\text { CHECKSHEET. }\end{array}$ & & & \\
\hline
\end{tabular}




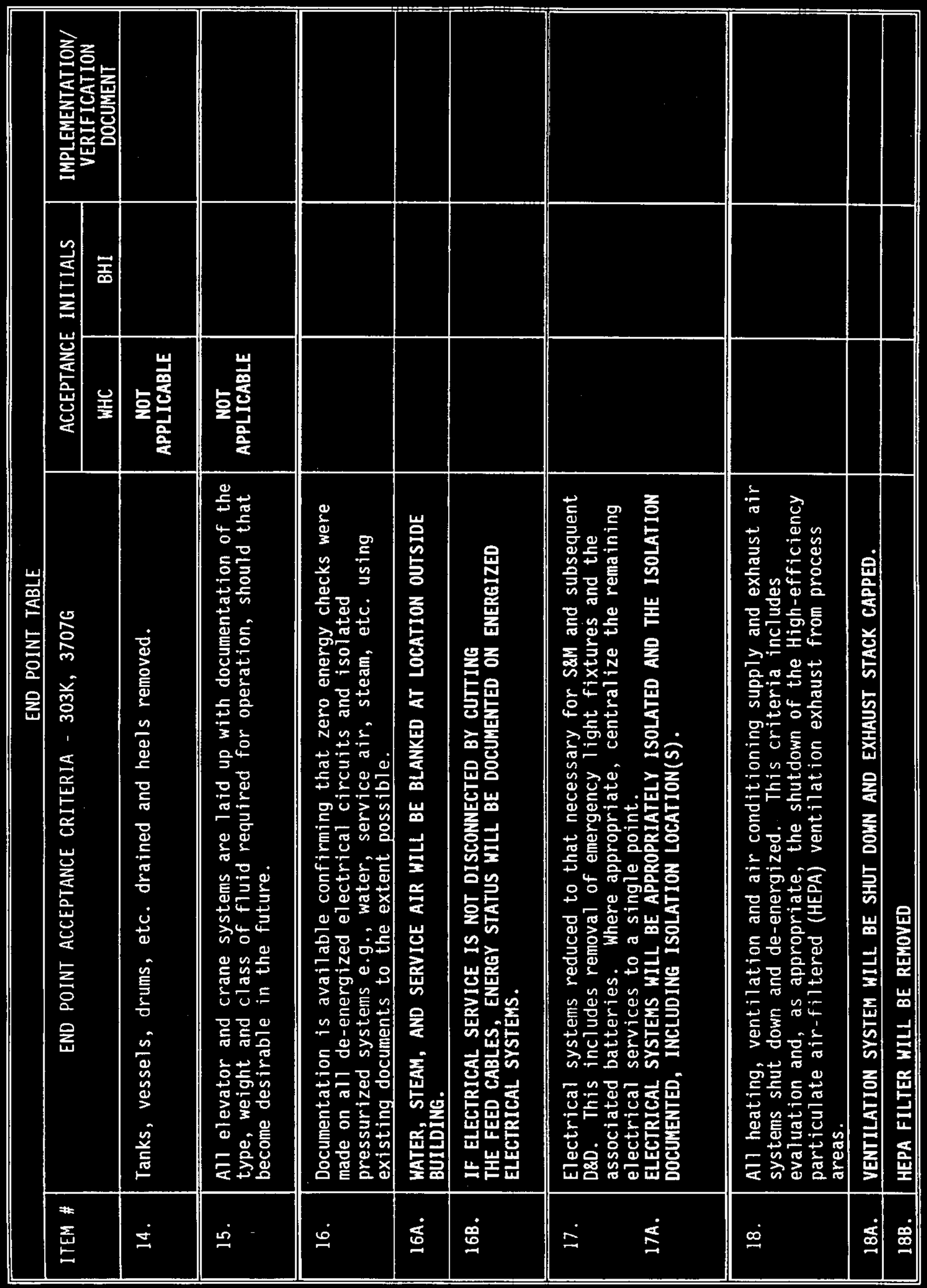




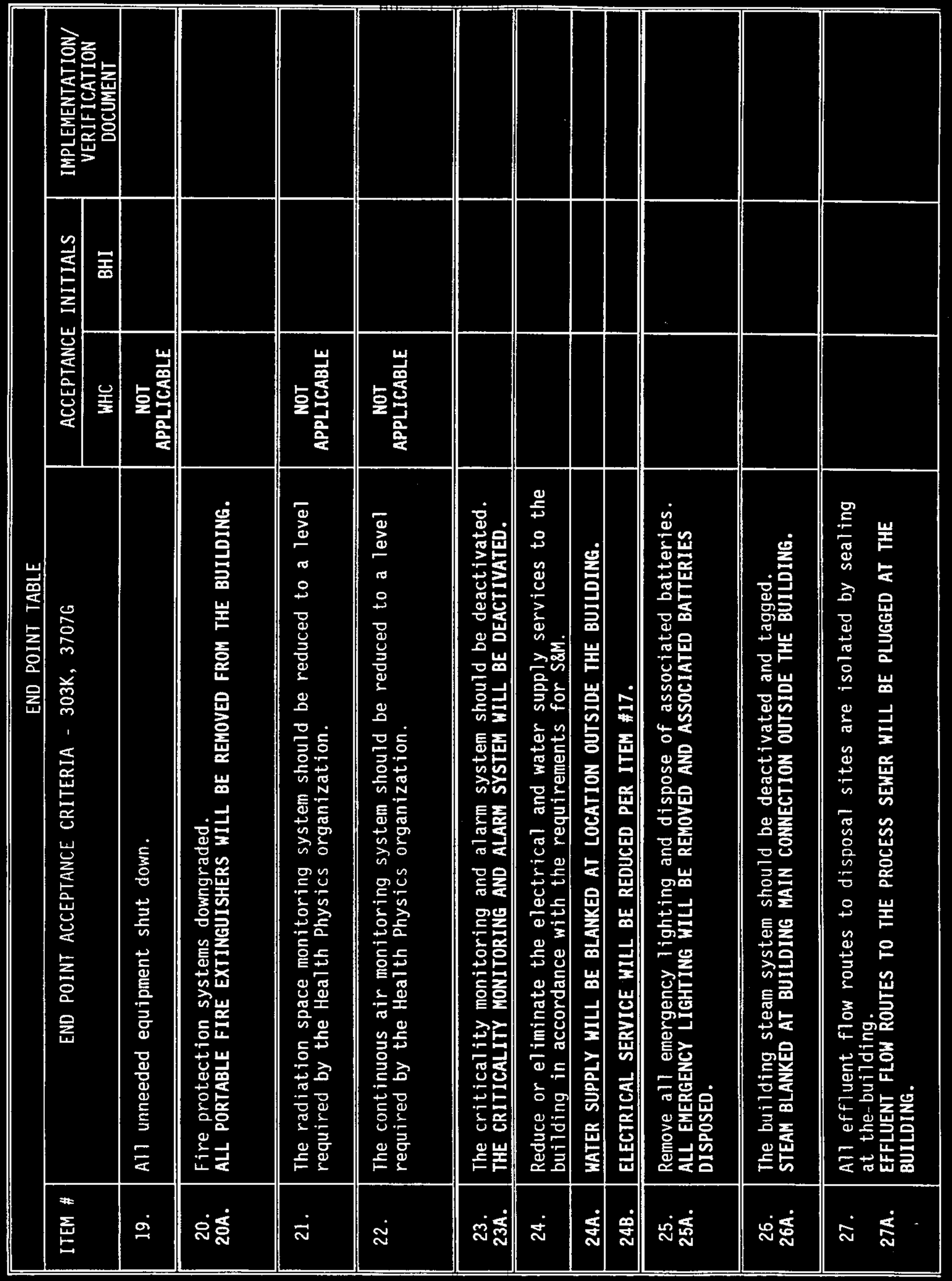




\begin{tabular}{|c|c|c|c|c|}
\hline \multicolumn{5}{|c|}{ END POINT TABLE } \\
\hline \multirow[t]{2}{*}{ ITEM \# } & \multirow[t]{2}{*}{ END POINT ACCEPTANCE CRITERIA - 303K, 3707G } & \multicolumn{2}{|c|}{ ACCEPTANCE INITIALS } & \multirow{2}{*}{$\begin{array}{l}\text { IMPLEMENTATION/ } \\
\text { VERIF ICATION } \\
\text { DOCUMENT }\end{array}$} \\
\hline & & WHC & BHI & \\
\hline 28 . & $\begin{array}{l}\text { A11 building penetrations, i.e., louvers, open piping, etc. shall be } \\
\text { closed off to prevent bird and animal intrusion. } \\
\text { BUILDING PENETRATIONS WILL BE CLOSED OFF TO PREVENT BIRD AND ANIMAL } \\
\text { INTRUSION. }\end{array}$ & & & \\
\hline 29. & $\begin{array}{l}\text { A11 systems that were open to facilitate deactivation and could } \\
\text { present a radiological and/or an industrial safety problem if left } \\
\text { open must be adequately closed off. } \\
\text { ALL OPEN SYSTEMS PRESENTING RADIOLOGICAL AND/OR INDUSTRIAL. SAFETY } \\
\text { PROBLEM WILL BE ADEQUATELY CLOSED OFF. }\end{array}$ & & & \\
\hline 30. & $\begin{array}{l}\text { Roof leaks repaired and suspect areas reviewed. } \\
\text { ROOF WILL BE INSPECTED WITHIN SIX MONTHS OF TURNOVER DATE AND } \\
\text { NECESSARY REPAIRS MADE SO THAT FURTHER MAINTENANCE WILL NOT BE } \\
\text { EXPECTED FOR } 5 \text { YEARS. }\end{array}$ & & & \\
\hline 31. & Office furniture and supplies removed. & $\begin{array}{c}\text { NOT } \\
\text { APPLICABLE }\end{array}$ & & \\
\hline 32. & $\begin{array}{l}\text { Unnecessary parts, tools, and portable equipment removed. } \\
\text { ALL WILL BE REMOVED. }\end{array}$ & & & \\
\hline 33. & Janitorial supplies and unattached hazardous materials removed. & $\begin{array}{l}\text { NOT } \\
\text { APPLICABLE }\end{array}$ & & \\
\hline $\begin{array}{l}34 . \\
344 .\end{array}$ & $\begin{array}{l}\text { Loose and } \backslash \text { or damaged asbestos removed or stabilized. } \\
\text { LOOSE AND YOR DAMAGED ASBESTOS WILL BE REMOVED OR STABILIZED. } \\
\text { ASBESTOS CONDITION ASSESSWENT WILL BE UPDATED WITHIN THREE MONTHS OF } \\
\text { TURNOVER. }\end{array}$ & & & \\
\hline 35. & $\begin{array}{l}\text { Perform good housekeeping in all zones and areas in and around the } \\
\text { facility. } \\
\text { GOOD HOUSEKEEPING WILL BE PERFORMED IN ALL ZONES AND AREAS IN AND } \\
\text { AROUND FACILITY. }\end{array}$ & & & \\
\hline
\end{tabular}




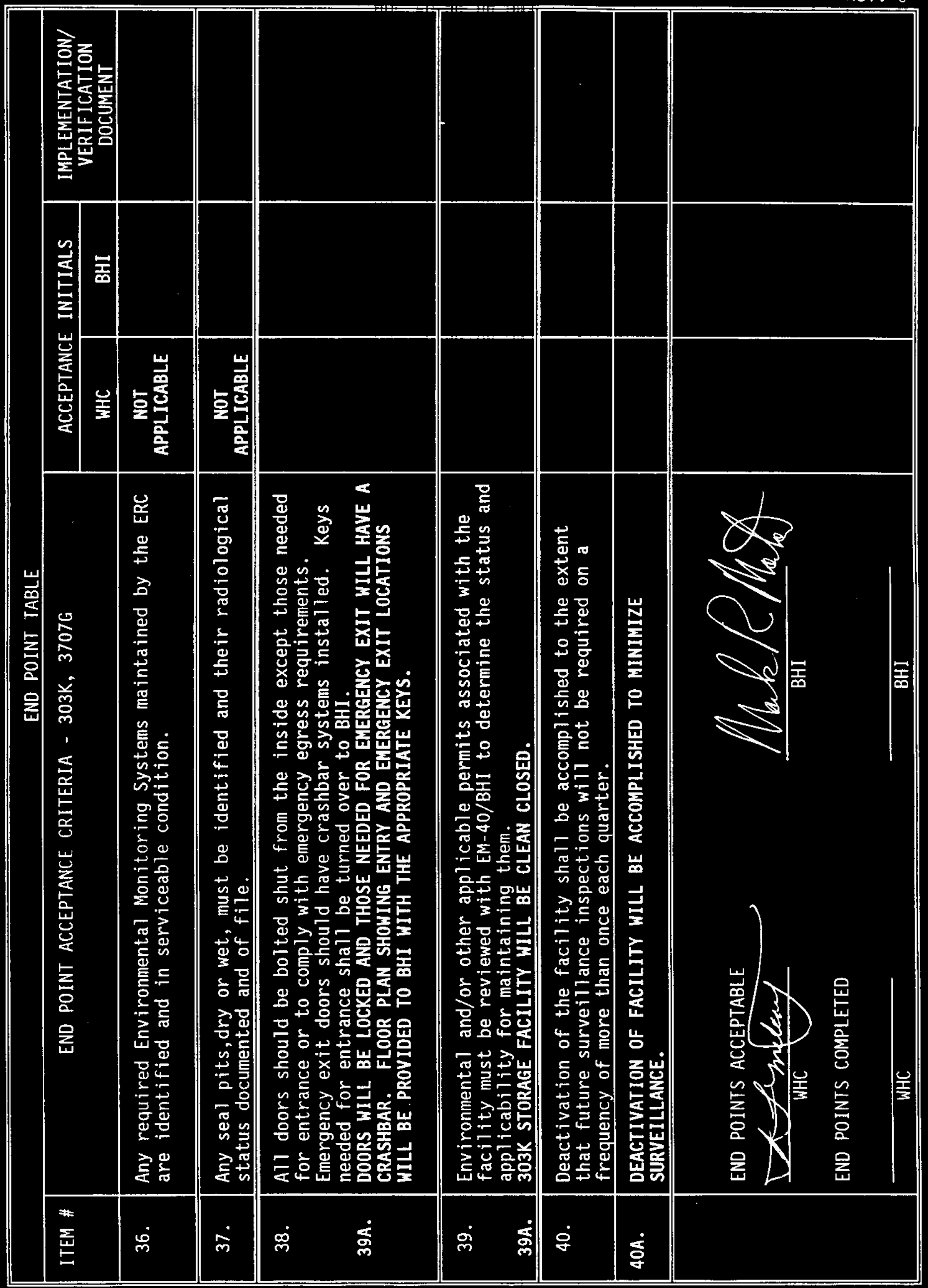




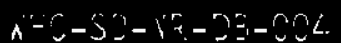

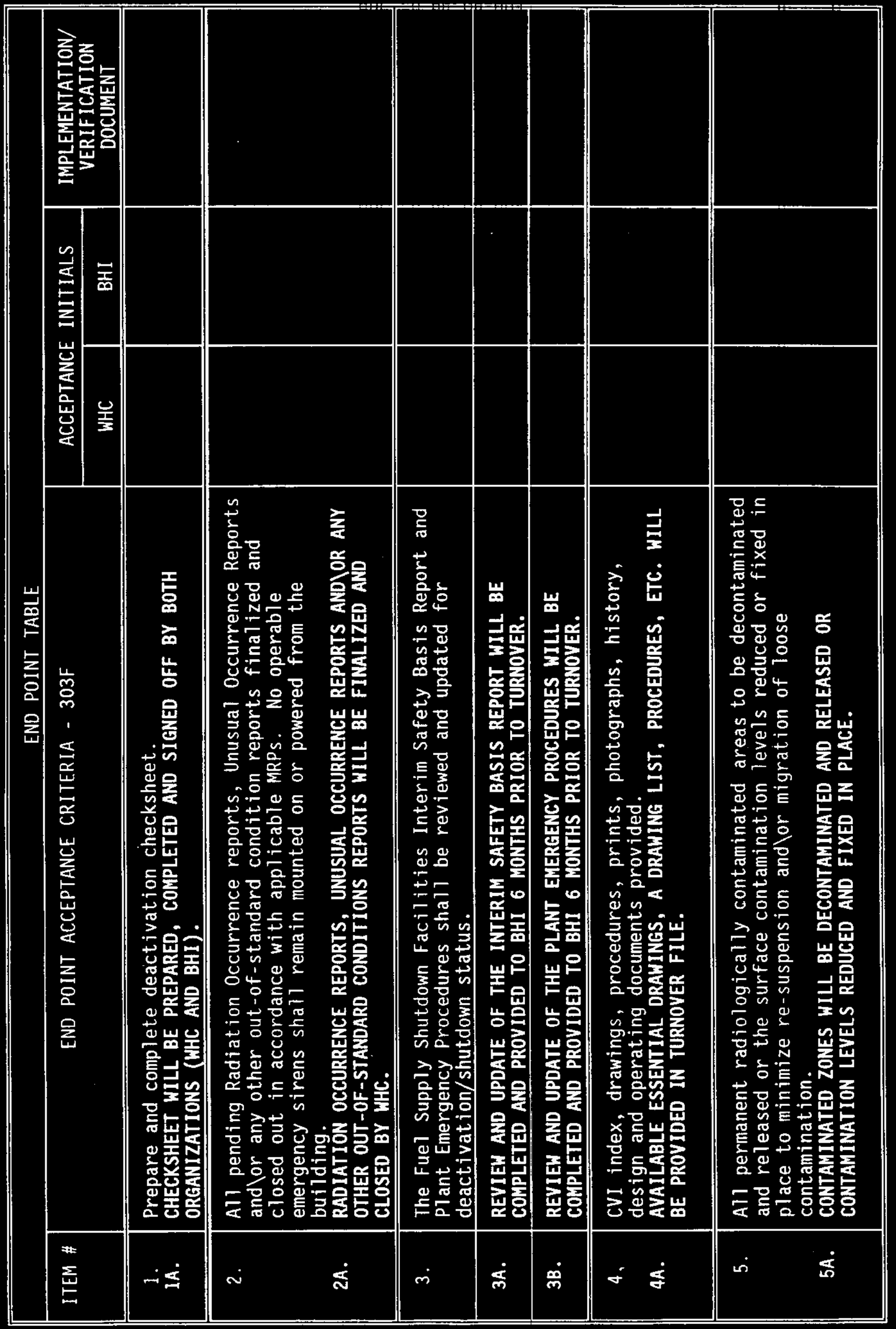




\begin{tabular}{|c|c|c|c|c|}
\hline \multicolumn{5}{|c|}{ END POINT TABLE } \\
\hline \multirow[t]{2}{*}{ ITEM \# } & \multirow[t]{2}{*}{ END POINT ACCEPTANCE CRITERIA - 303F } & \multicolumn{2}{|c|}{ ACCEPTANCE INITIALS } & \multirow{2}{*}{$\begin{array}{l}\text { IMPLEMENTATION/ } \\
\text { VERIFICATION } \\
\text { DOCUMENT }\end{array}$} \\
\hline & & WHC & $\mathrm{BHI}$ & \\
\hline 6. & $\begin{array}{l}\text { Full compliance with Hanford Site Radiological Control Manual must } \\
\text { be assured, especially as it pertains to radiological post ing. } \\
\text { COMPLIANCE WITH HANFORD SITE RADIOLOGICAL CONTROL MANUAL WILL BE } \\
\text { CONFIRMED BY HAVING } 300 \text { AREA RADIOLOGICAL CONTROL PERFORM AN } \\
\text { ASSESSMENT AND WHC WILL CORRECT ALL NOTED DISCREPANCIES. }\end{array}$ & & & \\
\hline 7 . & $\begin{array}{l}\text { Temporary radiologically posted areas are cleaned up and } \\
\text { radiologically released. } \\
\text { TEMPORARY RADIOLOGICALLY POSTED AREAS WILL BE CLEANED UP AND } \\
\text { RELEASED. }\end{array}$ & & & \\
\hline 8. & $\begin{array}{l}\text { A1l stored radioactive and mixed waste removed. } \\
\text { RADIOACTIVE AND MIXED WASTE WILL BE REMOVED. }\end{array}$ & & & \\
\hline 9. & $\begin{array}{l}\text { Al1 hazardous materials used for deactivation and cleanup work must } \\
\text { be collected and disposed of in accordance with requirements. } \\
\text { ALL HAZARDOUS MATERIALS USED FOR DEACTIVATION AND CLEANUP WILL BE } \\
\text { REMOVED. }\end{array}$ & & & \\
\hline 10. & $\begin{array}{l}\text { A11 unattached hazardous materials(i.e., loose friable asbestos, } \\
\text { lead. mercury, etc.) must be removed from the facility and disposed } \\
\text { of in accordance with requirements. } \\
\text { ALL UNATTACHED HAZARDOUS MATERIALS WILL BE REMOVED. }\end{array}$ & & & \\
\hline 11 & $\begin{array}{l}\text { Attached hazardous materials located, ident ified, quant if ied, } \\
\text { labelled as necessary, and recorded. } \\
\text { ATTACHED HAZARDOUS MATERIALS WILL BE LOCATED, IDENTIFIED, } \\
\text { QUANTIFIED, LABELLED AS NECESSARY AND RECORDED. }\end{array}$ & & & \\
\hline 12. & $\begin{array}{l}\text { Al1 reactor fuel elements and } \backslash \text { or other source and special materials } \\
\text { must be removed from the building. }\end{array}$ & $\begin{array}{l}\text { NOT } \\
\text { APPLICABLE }\end{array}$ & & \\
\hline 13 & $\begin{array}{l}\text { Final radiological status surveys available. } \\
\text { FINAL RADIOLOGICAL SURVEYS WILL BE INCLUDED WITH THE DEACTIVATION } \\
\text { CHECKSHEET. }\end{array}$ & & & \\
\hline 14. & Tanks, vessels, drums, etc. drained and heels removed. & & & \\
\hline
\end{tabular}




\begin{tabular}{|c|c|c|c|c|}
\hline \multicolumn{5}{|c|}{ END POINT TABLE } \\
\hline \multirow[t]{2}{*}{ ITEM \# } & \multirow[t]{2}{*}{ END POINT ACCEPTANCE CRITERIA - 303F } & \multicolumn{2}{|c|}{ ACCEPTANCE INITIALS } & \multirow{2}{*}{$\begin{array}{l}\text { IMPLEMENTATION/ } \\
\text { VERIFICATION } \\
\text { DOCUMENT }\end{array}$} \\
\hline & & WHC & BHI & \\
\hline $14 A$. & ALL TANKS, VESSELS, DRUMS, ETC. WILL BE DRAINED AND HEELS REMOVED. & & & \\
\hline 148. & WATS AREA WITHIN THE BUILDING WILL BE CLEAN CLOSED. & & & \\
\hline 15. & $\begin{array}{l}\text { All elevator and crane systems are laid up with documentation of the } \\
\text { type, weight and class of fluid required for operation, should that } \\
\text { become desirable in the future. }\end{array}$ & $\begin{array}{l}\text { NOT } \\
\text { APPLICABLE }\end{array}$ & & \\
\hline 16. & $\begin{array}{l}\text { Documentation is available confirming that zero energy checks were } \\
\text { made on all de-energized electrical circuits and isolated } \\
\text { pressurized systems e.g., water, service air, steam, etc. using } \\
\text { existing documents to the extent possible. }\end{array}$ & & & \\
\hline $16 \mathrm{~A}$. & $\begin{array}{l}\text { WATER, STEAM, AND SERVICE AIR WILL BE BLANKED AT LOCATION OUTSIDE } \\
\text { BUILDING. }\end{array}$ & & & \\
\hline 168. & $\begin{array}{l}\text { IF ELECTRICAL SERVICE IS NOT DISCONNECTED BY CUTTING THE FEED } \\
\text { CABLES, ENERGY STATUS WILL BE DOCUMENTED ON ENERGIZED ELECTRICAL } \\
\text { SYSTEMS. }\end{array}$ & & & \\
\hline $17 \mathrm{~A}$. & $\begin{array}{l}\text { Electrical systems reduced to that necessary for S\&M and subsequent } \\
\text { D\&D. This includes removal of emergency light fixtures and the } \\
\text { associated batteries. Where appropriate, centralize the remaining } \\
\text { electrical services to a single point. } \\
\text { ELECTRICAL SYSTEMS WILL BE APPROPRIATELY ISOLATED AND THE ISOLATION } \\
\text { DOCUMENTED, INCLUDING ISOLATION LOCATION(S). }\end{array}$ & & & \\
\hline 18. & $\begin{array}{l}\text { A11 heating, ventilation and air conditioning supply and exhaust air } \\
\text { systems shut down and de-energized. This criteria includes } \\
\text { evaluation and, as appropriate, the shutdown of the High-efficiency } \\
\text { particulate air-filtered (HEPA) ventilation exhaust from process } \\
\text { areas. }\end{array}$ & $\begin{array}{l}\text { NOT } \\
\text { APPLICABLE }\end{array}$ & & \\
\hline 19. & All unneeded equipment shut down. & $\begin{array}{c}\text { NOT } \\
\text { APPLICABLE }\end{array}$ & & \\
\hline
\end{tabular}




\begin{tabular}{|c|c|c|c|c|}
\hline \multicolumn{5}{|c|}{ END POINT TABLE } \\
\hline \multirow[t]{2}{*}{ ITEM \# } & \multirow[t]{2}{*}{ END POINT ACCEPTANCE CRITERIA - 303F } & \multicolumn{2}{|c|}{ ACCEPTANCE INITIALS } & \multirow{2}{*}{$\begin{array}{l}\text { IMPLEMENTATION/ } \\
\text { VERIFICATION } \\
\text { DOCUMENT }\end{array}$} \\
\hline & & WHC & BHI & \\
\hline 20 & $\begin{array}{l}\text { Fire protection systems downgraded. } \\
\text { ALL PORTABLE FIRE EXTINGUISHERS WILL BE REWOVED FRON THE BUILDING. }\end{array}$ & & & \\
\hline 21. & $\begin{array}{l}\text { The radiation space monitoring system should be reduced to a level } \\
\text { required by the Health Physics organization. }\end{array}$ & $\begin{array}{l}\text { NOT } \\
\text { APPLICABLE }\end{array}$ & & \\
\hline 22. & $\begin{array}{l}\text { The continuous air monitoring system should be reduced to a level } \\
\text { required by the Health Physics organization. }\end{array}$ & $\begin{array}{l}\text { NOT } \\
\text { APPLICABLE }\end{array}$ & & \\
\hline 23. & The criticality monitoring and alarm system should be deactivated. & $\begin{array}{l}\text { NOT } \\
\text { APPLICABLE }\end{array}$ & & \\
\hline 24. & $\begin{array}{l}\text { Reduce or eliminate the electrical, service air, and water supply } \\
\text { services to the building in accordance with the requirements for } \\
\text { S\&M. }\end{array}$ & & & \\
\hline $24 A$. & WATER SUPPLY WILL BE BLANKED AT LOCATION OUTSIDE THE BUILDING. & & & \\
\hline 24B. & ELECTRICAL SERVICE WILL BE REDUCED PER ITEM \#17. & & & \\
\hline $24 C$. & SERVICE AIR WILL BE BLANKED AT BUILDING ENTRANCE. & & & \\
\hline $\begin{array}{l}25 \\
25 A\end{array}$ & $\begin{array}{l}\text { Remove all emergency } 1 \text { ighting and dispose of associated batteries. } \\
\text { ALL. EMERGENCY LIGHTING WILL BE REMOVED AND ASSOCIATED BATTERIES } \\
\text { DISPOSED. }\end{array}$ & & & \\
\hline $\begin{array}{l}26 . \\
26 A\end{array}$ & $\begin{array}{l}\text { The building steam system should be deactivated and tagged. } \\
\text { STEAM BLANKED AT BUILDING MAIN CONNECTION OUTSIDE THE BUILOING. }\end{array}$ & & & \\
\hline 27. & $\begin{array}{l}\text { All effluent flow routes to disposal sites are isolated by sealing } \\
\text { at the building. } \\
\text { EFFLUENT FLOW ROUTES TO THE PROCESS SENER WILL BE PLUGGED AT THE } \\
\text { BUILDING. }\end{array}$ & & & \\
\hline
\end{tabular}




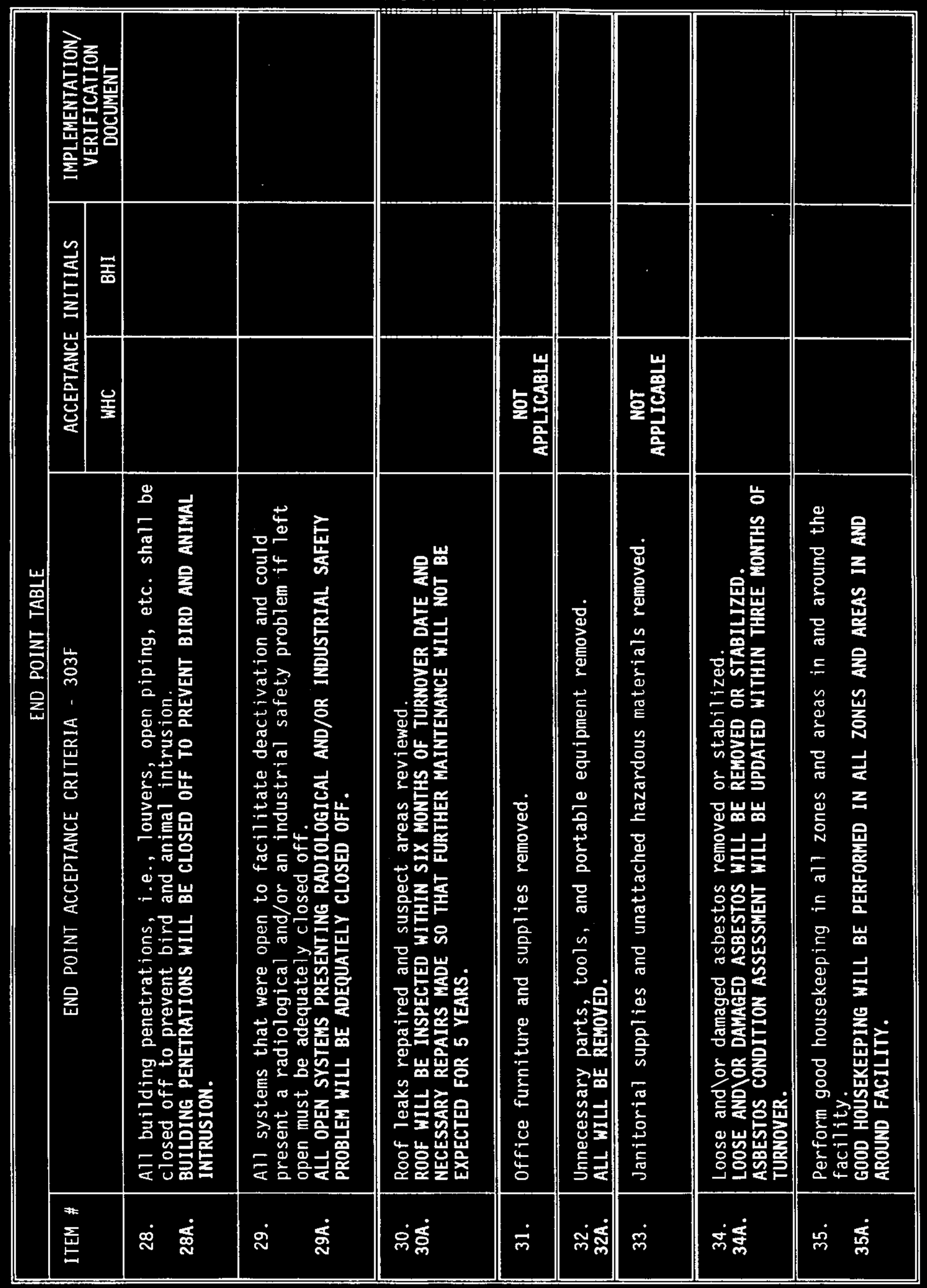


$A-2-j 2-1+23-0,0$

Rev. 0

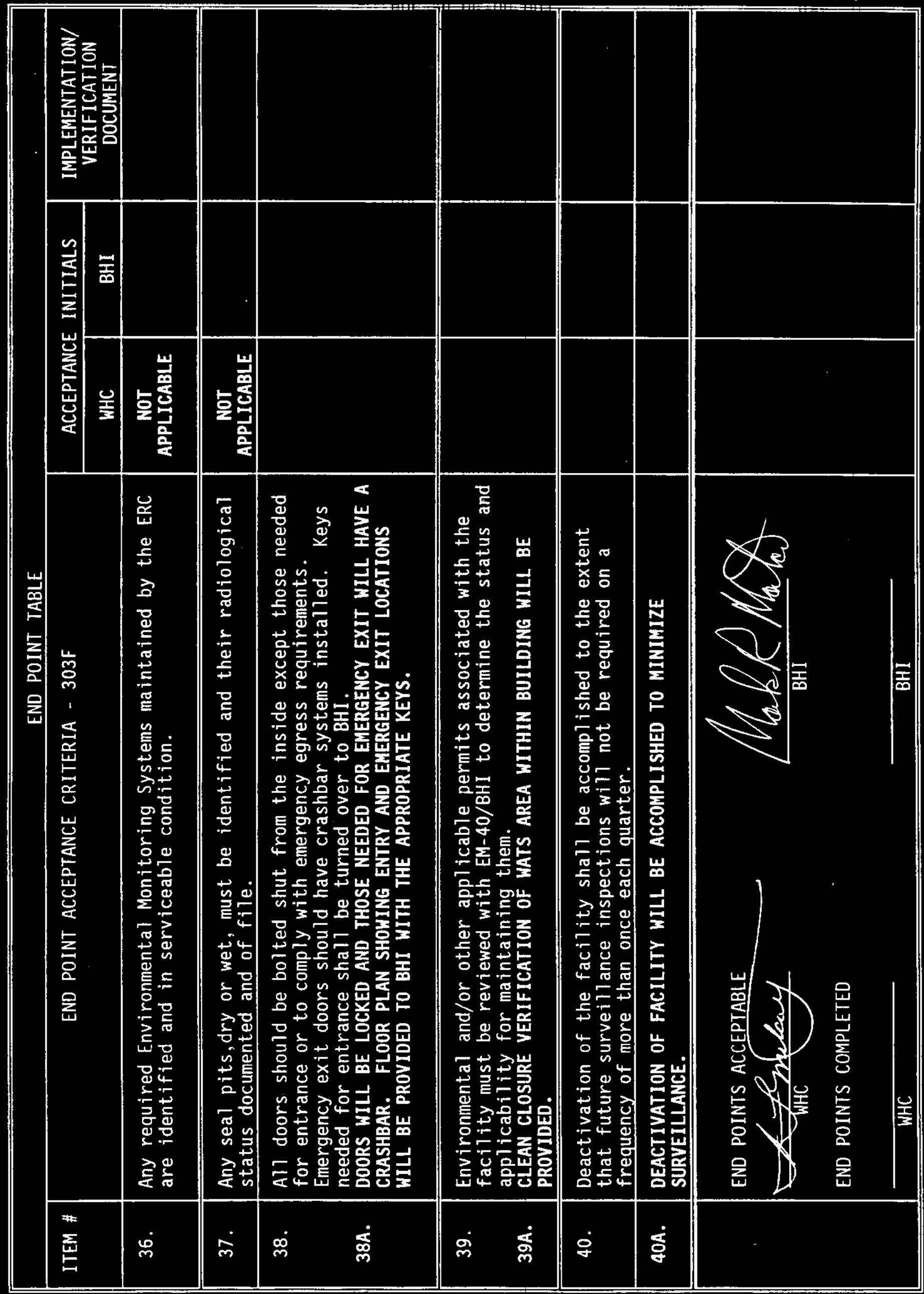


Rev. 0

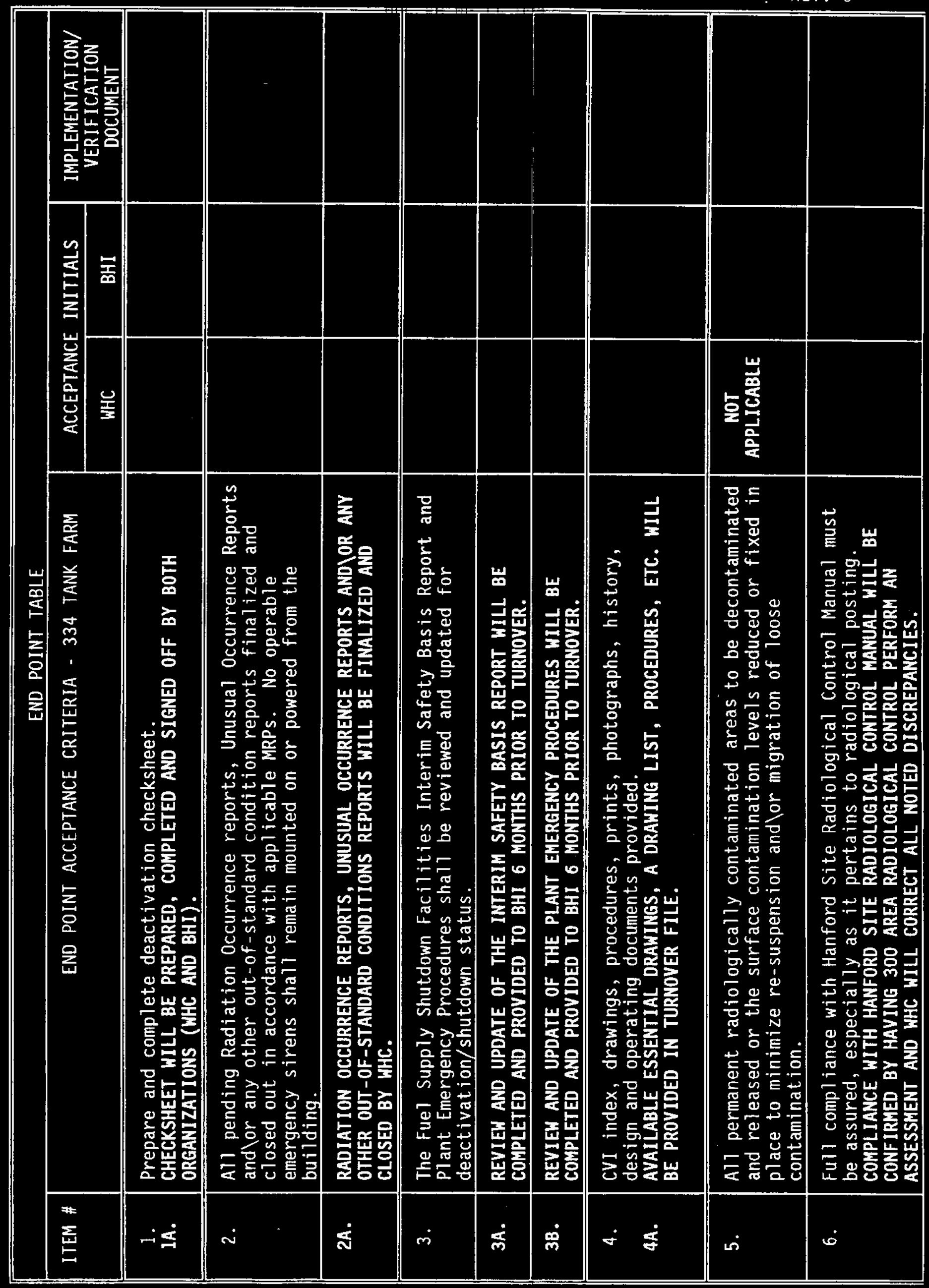




\begin{tabular}{|c|c|c|c|c|}
\hline \multicolumn{5}{|c|}{ END POINT TABLE } \\
\hline \multirow[t]{2}{*}{ ITEM \# } & \multirow[t]{2}{*}{ END POINT ACCEPTANCE CRITERIA - 334 TANK FARM } & \multicolumn{2}{|c|}{ ACCEPTANCE INITIALS } & \multirow{2}{*}{$\begin{array}{l}\text { IMPLEMENTATION } \\
\text { VERIFICATION } \\
\text { DOCUMENT }\end{array}$} \\
\hline & & WHC & BHI & \\
\hline 7. & $\begin{array}{l}\text { Temporary radiologically posted areas are cleaned up and } \\
\text { radiologically released. }\end{array}$ & $\begin{array}{l}\text { NOT } \\
\text { APPLICABLE }\end{array}$ & & \\
\hline 8. & A11 stored radioactive and mixed waste removed. & $\begin{array}{l}\text { NOT } \\
\text { APPLICABLE }\end{array}$ & & \\
\hline $\begin{array}{l}9 . \\
9 A .\end{array}$ & $\begin{array}{l}\text { A11 hazardous materials used for deactivation and cleanup work must } \\
\text { be collected and disposed of in accordance with requirements. } \\
\text { ALL. HAZARDOUS MATERIALS USED FOR DEACTIVATION AND CLEANUP WILL BE } \\
\text { REMOVED. }\end{array}$ & & & \\
\hline 10. & $\begin{array}{l}\text { All unattached hazardous materials(i.e., Toose friable asbestos, } \\
\text { lead. mercury, etc.) must be removed from the facility and disposed } \\
\text { of in accordance with requirements. }\end{array}$ & & & \\
\hline $10 A$. & ALL UNATTACHED HAZARDOUS MATERIALS WILL BE REMOVED. & & & \\
\hline 11 & $\begin{array}{l}\text { Attached hazardous materials located, identified, quantified, } \\
\text { labelled as necessary, and recorded. }\end{array}$ & & & \\
\hline $11 \mathrm{~A}$. & $\begin{array}{l}\text { ATTACHED HAZARDOUS MATERIALS WILL BE LOCATED, IDENTIFIED, } \\
\text { QUANTIFIED, LABELLED AS NECESSARY AND RECORDED. }\end{array}$ & & & \\
\hline 12. & $\begin{array}{l}\text { All reactor fuel elements and lor other source and special materials } \\
\text { must be removed from the building. }\end{array}$ & $\begin{array}{l}\text { NOT } \\
\text { APPLICABLE }\end{array}$ & & \\
\hline 13. & Final radiological status surveys available. & & & \\
\hline $13 A$. & $\begin{array}{l}\text { FINAL RADIOLOGICAL SURVEYS WILL BE INCLUDED WITH THE DEACTIVATION } \\
\text { CHECKSHEET. }\end{array}$ & & & \\
\hline \multirow[t]{2}{*}{14.} & Tanks, vessels, drums, etc. drained and heels removed. & & & \\
\hline & TANKS WILL BE DRAINED AND HEELS REMOVED: & & & \\
\hline $14 A$. & TANK 1 & & & \\
\hline $14 \mathrm{~B}$. & TANK 2 & & & \\
\hline $14 \mathrm{C}$. & TANK 3 & & & \\
\hline
\end{tabular}




\begin{tabular}{|c|c|c|c|c|}
\hline \multicolumn{5}{|c|}{ END POINT TABLE } \\
\hline \multirow[t]{2}{*}{ ITEM \# } & \multirow{2}{*}{ END POINT ACCEPTANCE CRITERIA - 334 TANK FARM } & \multicolumn{2}{|c|}{ ACCEPTANCE INITIALS } & \multirow{2}{*}{$\begin{array}{l}\text { IMPLEMENTATION/ } \\
\text { VERIF ICATION } \\
\text { DOCUMENT }\end{array}$} \\
\hline & & WHC & BHI & \\
\hline 15. & $\begin{array}{l}\text { A11 elevator and crane systems are laid up with documentation of the } \\
\text { type, weight and class of fluid required for operation, should that } \\
\text { become desirable in the future. }\end{array}$ & $\begin{array}{l}\text { NOT } \\
\text { APPLICABLE }\end{array}$ & & \\
\hline 16. & $\begin{array}{l}\text { Documentation is available confirming that zero energy checks were } \\
\text { made on all de-energized electrical circuits and isolated } \\
\text { pressurized systems e.g., water, service air, steam, etc. using } \\
\text { existing documents to the extent possible. }\end{array}$ & & & \\
\hline $16 \mathrm{~A}$. & WATER WILL BE BLANKED AT LOCATION OF MAIN SERVICE POINT. & & & \\
\hline 17. & $\begin{array}{l}\text { Electrical systems reduced to that necessary for } S \& M \text { and subsequent } \\
\text { D\&D. This includes removal of emergency light fixtures and the } \\
\text { associated batteries. Where appropriate, centralize the remaining } \\
\text { electrical services to a single point. }\end{array}$ & $\begin{array}{l}\text { NOT } \\
\text { APPLICABLE }\end{array}$ & & \\
\hline 18. & $\begin{array}{l}\text { All heating, ventilation and air conditioning supply and exhaust air } \\
\text { systems shut down and de-energized. This criteria includes } \\
\text { evaluation and, as appropriate, the shutdown of the High-efficiency } \\
\text { particulate air-filtered (HEPA) ventilation exhaust from process } \\
\text { areas. }\end{array}$ & $\begin{array}{l}\text { NOT } \\
\text { APPLICABLE }\end{array}$ & & \\
\hline 19. & A11 unneeded equipment shut down. & $\begin{array}{l}\text { NOT } \\
\text { APPLICABLE }\end{array}$ & & \\
\hline 20. & Fire protection systems downgraded. & $\begin{array}{l}\text { NOT } \\
\text { APPLICABLE }\end{array}$ & & \\
\hline 21. & $\begin{array}{l}\text { The radiation space monitoring system should be reduced to a level } \\
\text { required by the Health Physics organization. }\end{array}$ & $\begin{array}{l}\text { NOT } \\
\text { APPLICABLE }\end{array}$ & & \\
\hline 22. & $\begin{array}{l}\text { The continuous air monitoring system should be reduced to a level } \\
\text { required by the Health Physics organization. }\end{array}$ & $\begin{array}{l}\text { NOT } \\
\text { APPLICABLE }\end{array}$ & & \\
\hline
\end{tabular}




\begin{tabular}{|c|c|c|c|c|}
\hline \multicolumn{5}{|c|}{ END POINT TABLE } \\
\hline \multirow[t]{2}{*}{ ITEM \# } & \multirow[t]{2}{*}{ END POINT ACCEPTANCE CRITERIA - 334 TANK FARM } & \multicolumn{2}{|c|}{ ACCEPTANCE INITIALS } & \multirow{2}{*}{$\begin{array}{l}\text { IMPLEMENTATION } \\
\text { VERIFICATION } \\
\text { DOCUMENT }\end{array}$} \\
\hline & & WHC & BHI & \\
\hline 23. & The criticality monitoring and alarm system should be deactivated. & $\begin{array}{l}\text { NOT } \\
\text { APPLICABLE }\end{array}$ & & . \\
\hline 24. & $\begin{array}{l}\text { Reduce or eliminate the electrical, service air, and water supply } \\
\text { services to the building in accordance with the requirements for } \\
\text { S\&M. }\end{array}$ & & & \\
\hline 24A. & WATER SUPPLY WILL BE BLANKED AT LOCATION OF MAIN SERVICE POINT. & & & \\
\hline 25 . & Remove all emergency lighting and dispose of associated batteries. & $\begin{array}{l}\text { NOT } \\
\text { APPLICABLE }\end{array}$ & & \\
\hline 26. & The building steam system should be deactivated and tagged. & $\begin{array}{l}\text { NOT } \\
\text { APPLICABLE }\end{array}$ & & \\
\hline 27. & $\begin{array}{l}\text { All effluent flow routes to disposal sites are isolated by sealing } \\
\text { at the building. }\end{array}$ & & & \\
\hline $27 A$. & PROCESS SENER FLON ROUTE WILL BE ISOLATED BY SEALING. & & & \\
\hline 28. & $\begin{array}{l}\text { All building penetrations, i.e., louvers, open piping, etc. shall be } \\
\text { closed off to prevent bird and animal intrusion. }\end{array}$ & $\begin{array}{l}\text { NOT } \\
\text { APPLICABLE }\end{array}$ & & \\
\hline $\begin{array}{l}29 . \\
29 A .\end{array}$ & $\begin{array}{l}\text { A11 systems that were open to facilitate deactivation and could } \\
\text { present a radiological and/or an industrial safety problem if left } \\
\text { open must be adequately closed off. } \\
\text { ALL OPEN SYSTEMS PRESENTING RADIOLOGICAL AND/OR INDUSTRIAL SAFETY } \\
\text { PROBLEM WILL BE ADEQUATELY CLOSED OFF. }\end{array}$ & & & \\
\hline 30. & Roof leaks repaired and suspect areas reviewed. & $\begin{array}{l}\text { NOT } \\
\text { APPLICABLE }\end{array}$ & & \\
\hline 31. & Office furniture and supplies removed. & $\begin{array}{l}\text { NOT } \\
\text { APPLICABLE }\end{array}$ & & \\
\hline 32. & Unnecessary parts, tools, and portable equipment removed. & $\begin{array}{l}\text { NOT } \\
\text { APPLICABLE }\end{array}$ & & \\
\hline
\end{tabular}




\begin{tabular}{|c|c|c|c|c|}
\hline \multicolumn{5}{|c|}{ END POINT TABLE } \\
\hline \multirow[t]{2}{*}{ ITEM \# } & \multirow{2}{*}{ END POINT ACCEPTANCE CRITERIA - 334 TANK FARM } & \multicolumn{2}{|c|}{ ACCEPTANCE INITIALS } & \multirow{2}{*}{$\begin{array}{l}\text { IMPLEMENTATION } \\
\text { VERIF ICATION } \\
\text { DOCUMENT }\end{array}$} \\
\hline & & WHC & BHI & \\
\hline 33. & Janitorial supplies and unattached hazardous materials removed. & $\begin{array}{l}\text { NOT } \\
\text { APPLICABLE }\end{array}$ & & \\
\hline 34. & Loose and \or damaged asbestos removed or stabilized. & & & \\
\hline $34 A$. & $\begin{array}{l}\text { LOOSE AND \OR DAMAGED ASBESTOS WILL BE REMOVED OR STABILIZED. ASBESTOS } \\
\text { CONDITION ASSESSMENT WILL BE UPDATED WITHIN THREE MONTHS OF } \\
\text { TURNOVER. }\end{array}$ & & & \\
\hline 35 . & $\begin{array}{l}\text { Perform good housekeeping in all zones and areas in and around the } \\
\text { facility. }\end{array}$ & & & \\
\hline $35 A$. & $\begin{array}{l}\text { GOOD HOUSEKEEPING WILL BE PERFORMED IN ALL ZONES AND AREAS IN AND } \\
\text { AROUND FACILITY. }\end{array}$ & & & \\
\hline 36. & $\begin{array}{l}\text { Any required Environmental Monitoring Systems maintained by the ERC } \\
\text { are identified and in serviceable condition. }\end{array}$ & $\begin{array}{l}\text { NOT } \\
\text { APPLICABLE }\end{array}$ & & \\
\hline 37. & $\begin{array}{l}\text { Any seal pits, dry or wet, must be identified and their radiological } \\
\text { status documented and of file. }\end{array}$ & $\begin{array}{l}\text { NOT } \\
\text { APPLICABLE }\end{array}$ & & \\
\hline 38. & $\begin{array}{l}\text { A11 doors should be bolted shut from the inside except those needed } \\
\text { for entrance or to comply with emergency egress requirements. } \\
\text { Emergency exit doors should have crashbar systems installed. Keys } \\
\text { needed for entrance shall be turned over to BHI. }\end{array}$ & $\begin{array}{l}\text { NOT } \\
\text { APPLICABLE }\end{array}$ & & \\
\hline 39. & $\begin{array}{l}\text { Environmental and/or other applicable permits associated with the } \\
\text { facility must be reviewed with EM-40/BHI to determine the status and } \\
\text { applicability for maintaining them. } \\
\text { CLEAN CLOSURE VERIFICATION OF WATS AREA WILL BE PROVIOED. }\end{array}$ & $\begin{array}{l}\text { NOT } \\
\text { APPLICABLE }\end{array}$ & & \\
\hline 40. & $\begin{array}{l}\text { Deactivation of the facility shall be accomplished to the extent } \\
\text { that future surveillance inspections will not be required on a } \\
\text { frequency of more than once each quarter. }\end{array}$ & & & \\
\hline $40 A$. & $\begin{array}{l}\text { DEACTIVATION OF FACILITY WILL BE ACCOMPLISHED TO MINIMIZE } \\
\text { SURVEILLANCE. }\end{array}$ & & & \\
\hline
\end{tabular}




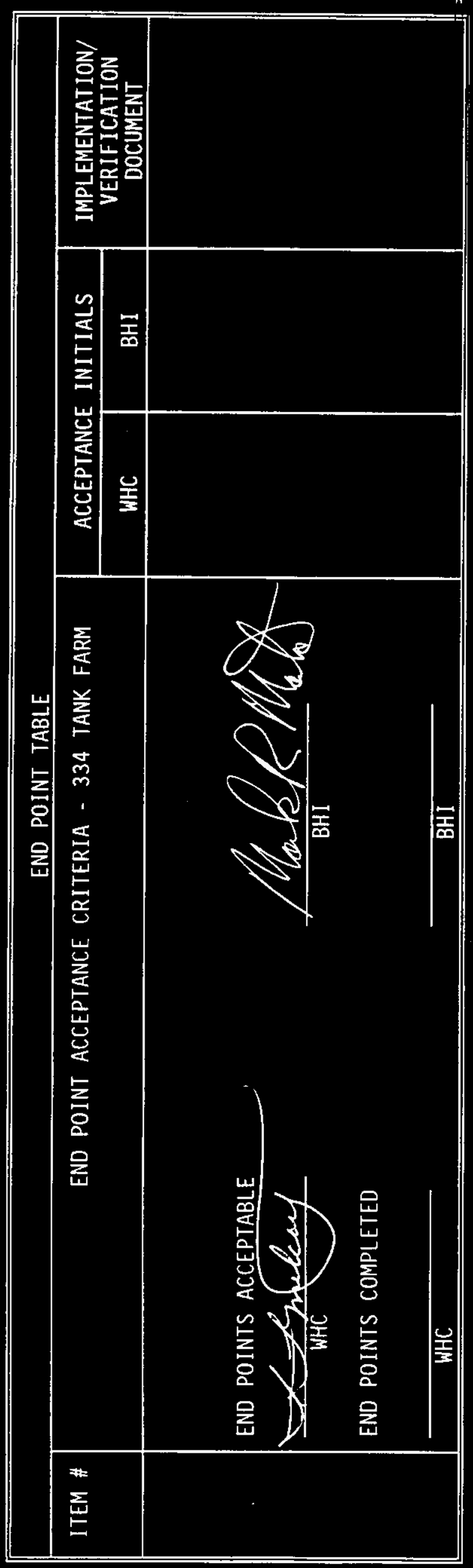

Rev. 0 


\begin{tabular}{|c|c|c|c|c|}
\hline \multicolumn{5}{|c|}{ END POINT TABLE } \\
\hline \multirow[t]{2}{*}{ ITEM \# } & \multirow[t]{2}{*}{ END POINT ACCEPTANCE CRITERIA - 311 TANK FARM } & \multicolumn{2}{|c|}{ ACCEPTANCE INITIALS } & \multirow{2}{*}{$\begin{array}{l}\text { IMPLEMENTATION/ } \\
\text { VERIFICATION } \\
\text { DOCUMENT }\end{array}$} \\
\hline & & WHC & BHI & \\
\hline $\begin{array}{l}1 . \\
1 A .\end{array}$ & $\begin{array}{l}\text { Prepare and complete deactivation checksheet. } \\
\text { CHECKSHEET WILL BE PREPARED, CONPLETED AND SIGNED OFF BY BOTH } \\
\text { ORGANIZATIONS (WHC AND BHI). }\end{array}$ & & & \\
\hline 2. & $\begin{array}{l}\text { Al1 pending Radiation Occurrence reports, Unusual Occurrence Reports } \\
\text { and } \backslash \text { or any other out-of-standard condition reports finalized and } \\
\text { closed out in accordance with applicable MRPs. No operable } \\
\text { emergency sirens shall remain mounted on or powered from the } \\
\text { building. }\end{array}$ & & & \\
\hline $2 A$. & $\begin{array}{l}\text { RADIATION OCCURRENCE REPORTS, UNUSUAL OCCURRENCE REPORTS AND } O \text { OR ANY } \\
\text { OTHER OUT-OF-STANDARD CONDITIONS REPORTS WILL BE FINALIZED AND } \\
\text { CLOSED BY WHC. }\end{array}$ & & & \\
\hline 3. & $\begin{array}{l}\text { The Fuel Supply Shutdown Facilities Interim Safety Basis Report and } \\
\text { Plant Emergency Procedures shall be reviewed and updated for } \\
\text { deactivation/shutdown status. }\end{array}$ & & & \\
\hline 3A. & $\begin{array}{l}\text { REVIEW AND UPDATE OF THE INTERIM SAFETY BASIS REPORT WILL BE } \\
\text { COMPLETED AND PROVIDED TO BHI } 6 \text { MONTHS PRIOR TO TURNOVER. }\end{array}$ & & & \\
\hline 3B. & $\begin{array}{l}\text { REVIEW AND UPDATE OF THE PLANT ENERGENCY PROCEDURES WILL BE } \\
\text { COMPLETED AND PROVIDED TO BHI } 6 \text { MONTHS PRIOR TO TURNOVER. }\end{array}$ & & & \\
\hline 4 . & $\begin{array}{l}\text { CVI index, drawings, procedures, prints, photographs, history, } \\
\text { design and operat ing documents provided. } \\
\text { AVAILABLE ESSENTIAL DRANINGS, A DRANING LIST, PROCEDURES, ETC. WILL } \\
\text { BE PROVIDED IN TURNOVER FILE. }\end{array}$ & & & \\
\hline 5. & $\begin{array}{l}\text { All permanent radiologically contaminated areas to be decontaminated } \\
\text { and released or the surface contamination levels reduced or fixed in } \\
\text { place to minimize re-suspension andlor migration of loose } \\
\text { contamination. }\end{array}$ & $\begin{array}{c}\text { NOT } \\
\text { APPLICABLE }\end{array}$ & & \\
\hline 6. & $\begin{array}{l}\text { Full compliance with Hanford Site Radiological Control Manual must } \\
\text { be assured, especially as it pertains to radiological post ing. } \\
\text { COMPLIANCE WITH HANFORD SITE RADIOLOGICAL CONTROL MANUAL WILL BE } \\
\text { CONF IRMED BY HAVING } 300 \text { AREA RADIOLOGICAL CONTROL PERFORM AN } \\
\text { ASSESSMENT AND WHC WILL CORRECT ALL NOTED DISCREPANCIES. }\end{array}$ & & & \\
\hline
\end{tabular}




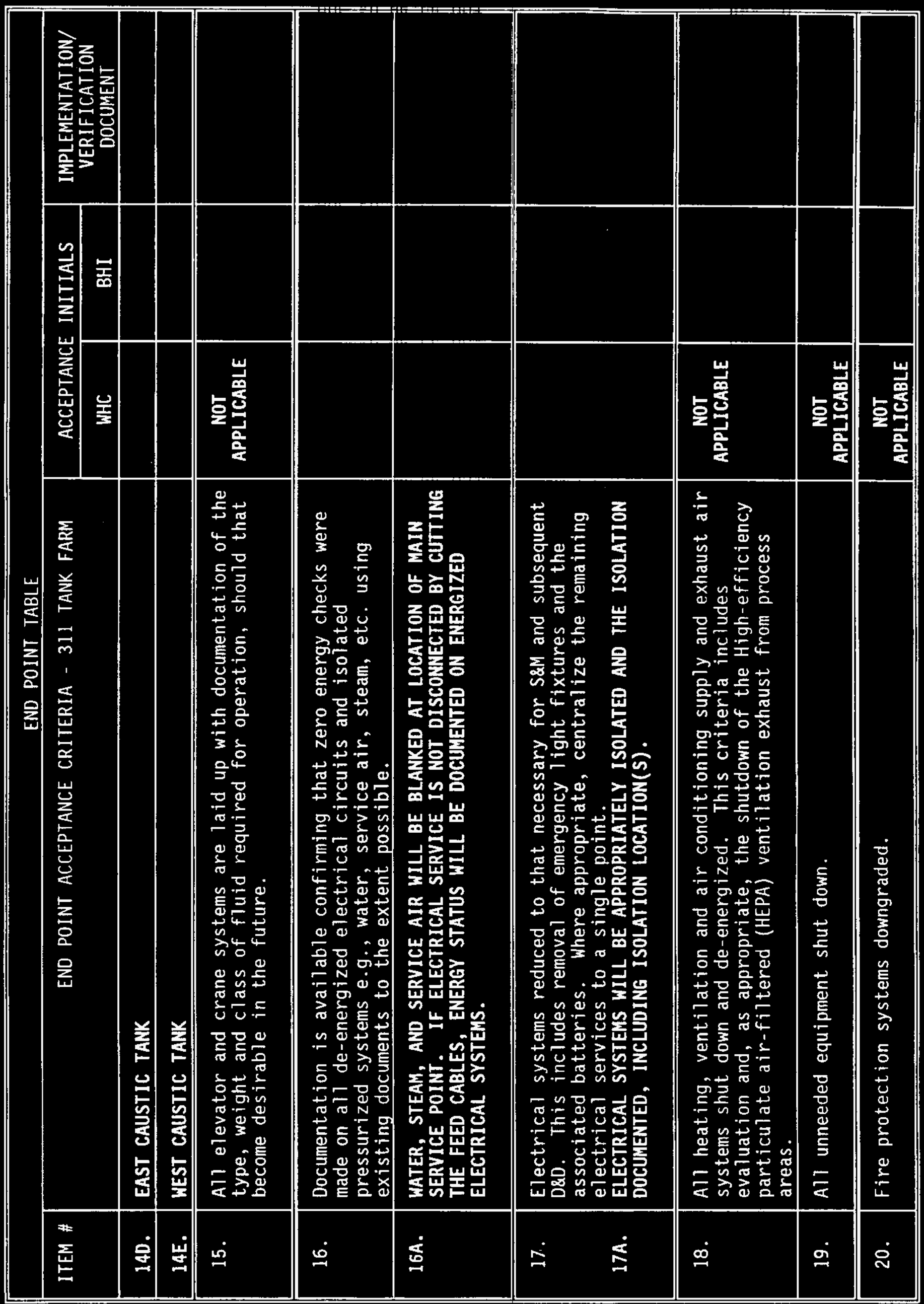




\begin{tabular}{|c|c|c|c|c|}
\hline \multicolumn{5}{|c|}{ END POINT TABLE } \\
\hline \multirow[t]{2}{*}{ ITEM \# } & \multirow[t]{2}{*}{ END POINT ACCEPTANCE CRITERIA - 311 TANK FARM } & \multicolumn{2}{|c|}{ ACCEPTANCE INITIALS } & \multirow{2}{*}{$\begin{array}{l}\text { IMPLEMENTATION/ } \\
\text { VERIFICATION } \\
\text { DOCUMENT }\end{array}$} \\
\hline & & WHC & BHI & \\
\hline 21. & $\begin{array}{l}\text { The radiation space monitoring system should be reduced to a level } \\
\text { required by the Health Physics organization. }\end{array}$ & $\begin{array}{l}\text { NOT } \\
\text { APPLICABLE }\end{array}$ & & \\
\hline 22. & $\begin{array}{l}\text { The continuous air monitoring system should be reduced to a level } \\
\text { required by the Health Physics organization. }\end{array}$ & $\begin{array}{l}\text { NOT } \\
\text { APPLICABLE }\end{array}$ & & \\
\hline 23. & The criticality monitoring and alarm system should be deactivated. & $\begin{array}{l}\text { NOT } \\
\text { APPLICABLE }\end{array}$ & & \\
\hline 24. & $\begin{array}{l}\text { Reduce or eliminate the electrical, service air, and water supply } \\
\text { services to the building in accordance with the requirements for } \\
\text { S\&M. }\end{array}$ & & & \\
\hline $24 \mathrm{~A}$. & WATER SUPPLY WILL BE BLANKED AT LOCATION OF MAIN SERVICE POINT. & & & \\
\hline 248. & ELECTRICAL SERVICE WILL BE REDUCED PER ITEM \#17. & & & \\
\hline 25. & Remove all emergency lighting and dispose of associated batteries. & $\begin{array}{c}\text { NOT } \\
\text { APPLICABLE }\end{array}$ & & \\
\hline 26. & The building steam system should be deactivated and tagged. & & & \\
\hline $26 A$. & THE STEAM SYSTEM WILL BE BLANKED AT MAIN SERVICE POINT. & & & \\
\hline 27. & $\begin{array}{l}\text { All effluent flow routes to disposal sites are isolated by sealing } \\
\text { at the building. }\end{array}$ & & & \\
\hline 27A. & PROCESS SEWER FLOW ROUTE WILL BE ISOLATED BY SEALING. & & & \\
\hline 28. & $\begin{array}{l}\text { All building penetrations, i.e., louvers, open piping, etc. shall be } \\
\text { closed off to prevent bird and animal intrusion. }\end{array}$ & $\begin{array}{l}\text { NOT } \\
\text { APPLICABLE }\end{array}$ & & \\
\hline
\end{tabular}

$\mathscr{8}$ 


\begin{tabular}{|c|c|c|c|c|}
\hline & & $\begin{array}{l}\text { 378YjI Tddy } \\
10 \mathrm{~N}\end{array}$ & 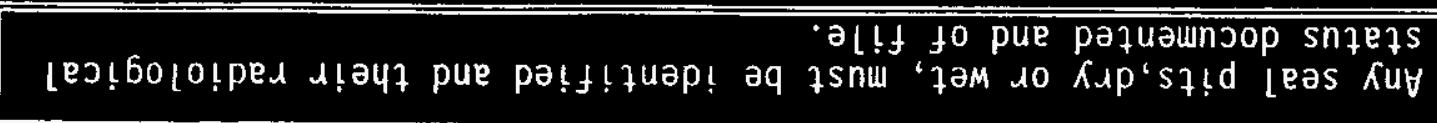 & $\cdot L \varepsilon$ \\
\hline & & $\begin{array}{l}378 y 917 d d y \\
10 N\end{array}$ & 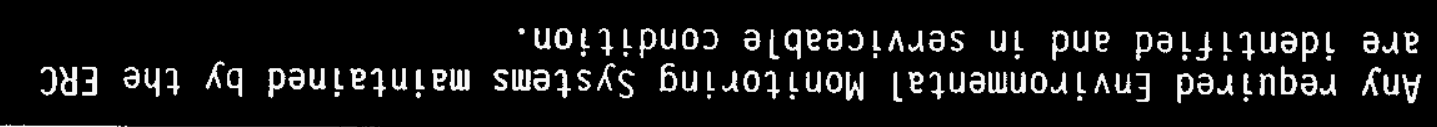 & $\cdot 9 \varepsilon$ \\
\hline & & & 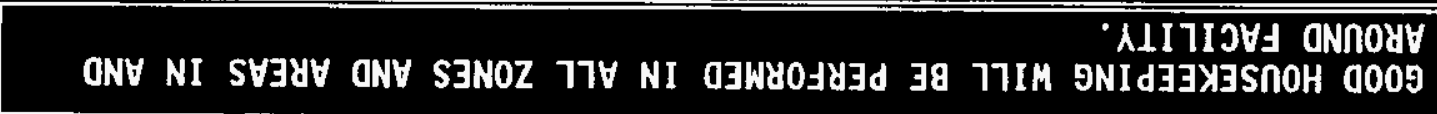 & • עa \\
\hline & & & 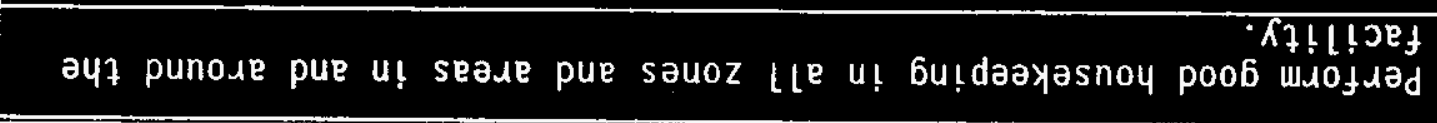 & $\cdot 9 \varepsilon$ \\
\hline & & & 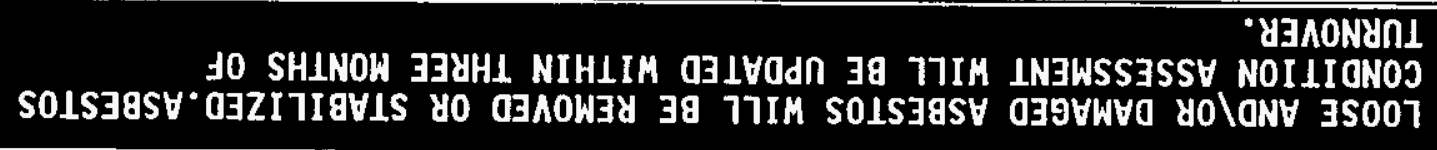 & $\cdot \forall \forall \varepsilon$ \\
\hline & & & -paz!l!qezs 10 panowad sozsaqse pafeurep $10 \backslash$ pue as007 & $\cdot \boldsymbol{\cdot}$ \\
\hline & & $\begin{array}{l}379 y 317 d d y \\
10 \mathrm{~N}\end{array}$ & 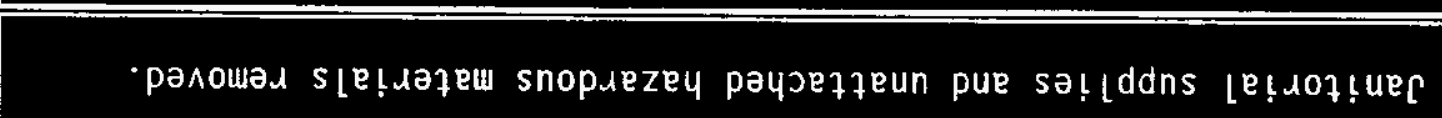 & • $\varepsilon \varepsilon$ \\
\hline & & 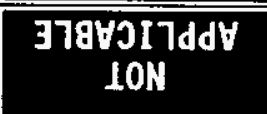 & 'panowal quawd!nba әlqequad pue 's[007 'squed kuessajauun & $\cdot 2 \varepsilon$ \\
\hline & & $\begin{array}{l}\text { 378VII } \\
\text { ION }\end{array}$ & 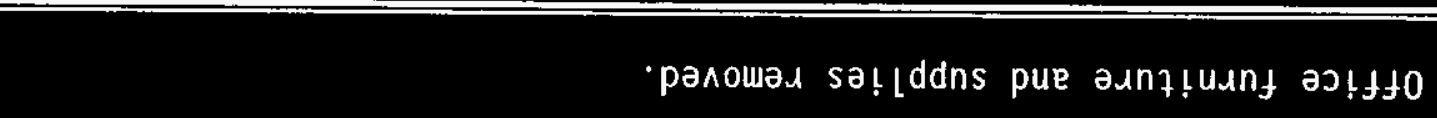 & - $[\varepsilon$ \\
\hline & & $\begin{array}{l}378 v 317 d d y \\
10 \mathrm{~N}\end{array}$ & 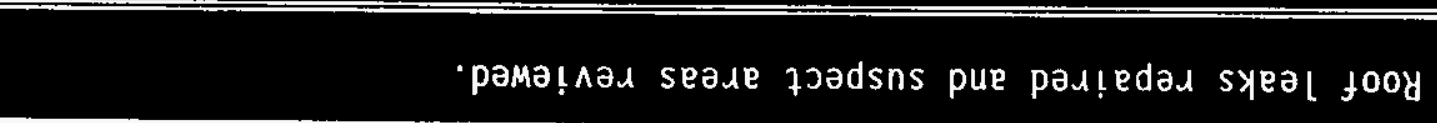 & . $0 \varepsilon$ \\
\hline & & & 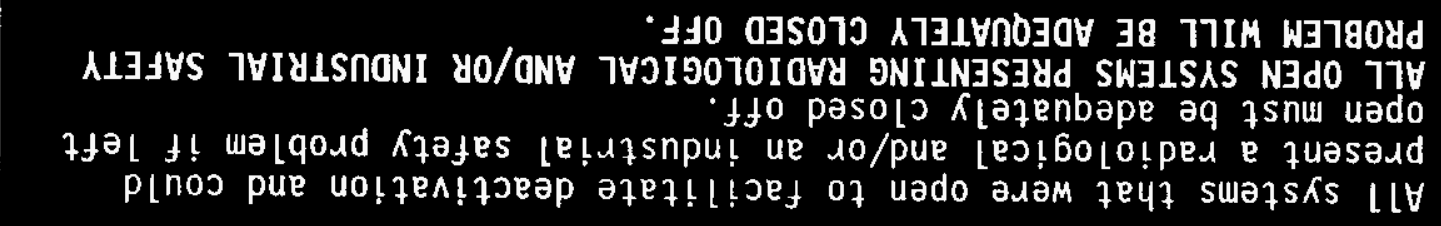 & $\begin{array}{l}\cdot \forall 62 \\
\cdot 62\end{array}$ \\
\hline \multirow{2}{*}{ 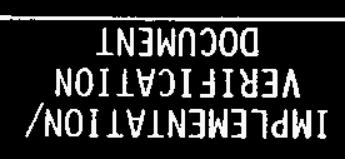 } & IH8 & JHM & \multirow[b]{2}{*}{ 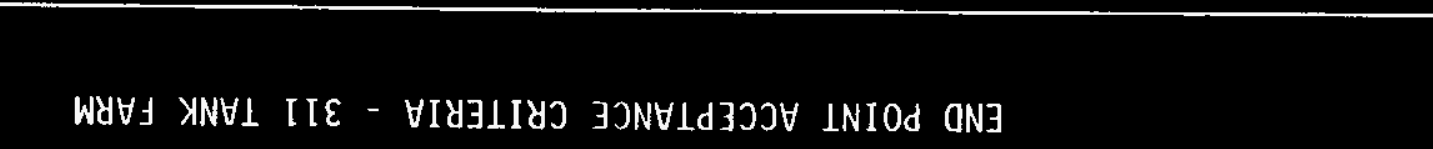 } & \multirow[b]{2}{*}{ \# WHL1 } \\
\hline & STHILINI & $\exists J N Y \perp d \exists J J Y$ & & \\
\hline
\end{tabular}




\begin{tabular}{|c|c|c|c|c|}
\hline \multicolumn{5}{|c|}{ END POINT TABLE } \\
\hline \multirow[t]{2}{*}{ ITEM \# } & \multirow[t]{2}{*}{ END POINT ACCEPTANCE CRITERIA - 311 TANK FARM } & \multicolumn{2}{|c|}{ ACCEPTANCE INITIALS } & \multirow{2}{*}{$\begin{array}{l}\text { IMPLEMENTATION/ } \\
\text { VERIFICATION } \\
\text { DOCUMENT }\end{array}$} \\
\hline & & WHC & BHI & \\
\hline 38. & $\begin{array}{l}\text { All doors should be bolted shut from the inside except those needed } \\
\text { for entrance or to comply with emergency egress requirements. } \\
\text { Emergency exit doors should have crashbar systems installed. Keys } \\
\text { needed for entrance shall be turned over to BHI. }\end{array}$ & $\begin{array}{c}\text { NoT } \\
\text { APPLICABLE }\end{array}$ & & \\
\hline $\begin{array}{l}39 . \\
39 A .\end{array}$ & $\begin{array}{l}\text { Environmental and/or other applicable permits associated with the } \\
\text { facility must be reviewed with EM-40/BHI to determine the status and } \\
\text { applicability for maintaining them. } \\
\text { CLEAN CLOSURE OF MATS SYSTEM TANKS WILL BE PROVIDED. }\end{array}$ & & & \\
\hline 40. & $\begin{array}{l}\text { Deactivation of the facility shall be accomplished to the extent } \\
\text { that future surveillance inspections will not be required on a } \\
\text { frequency of more than once each quarter. }\end{array}$ & & & \\
\hline \multirow[t]{3}{*}{ 40A. } & $\begin{array}{l}\text { DEACTIVATION OF FACILITY WILL BE ACCOMPLISHED TO MINIMIZE } \\
\text { SURVELEILLANCE. }\end{array}$ & & & \\
\hline & END POINTS ACCEPTABIE & & & \\
\hline & WHC & & & \\
\hline
\end{tabular}




\begin{tabular}{|c|c|c|c|c|}
\hline \multicolumn{5}{|c|}{ END POINT TABLE } \\
\hline \multirow[t]{2}{*}{ ITEM \# } & \multirow[t]{2}{*}{ END POINT ACCEPTANCE CRITERIA - OUTSIDE TRENCH SYSTEM } & \multicolumn{2}{|c|}{ ACCEPTANCE INITIALS } & \multirow{2}{*}{$\begin{array}{l}\text { IMPLEMENTATION/ } \\
\text { VERIFICATION } \\
\text { DOCUMENT } \\
\end{array}$} \\
\hline & & WHC & BHI & \\
\hline 1. & $\begin{array}{l}\text { Prepare and complete deactivation checksheet. } \\
\text { CHECASHEET WILL BE PREPARED, COMPLETED AND SIGNED OFF BY BOTH } \\
\text { ORGANIZATIONS (WHC AND BHI). }\end{array}$ & & & \\
\hline 2. & $\begin{array}{l}\text { All pending Radiation Occurrence reports, Unusual occurrence Reports } \\
\text { and } \backslash \text { or any other out-of-standard condition reports finalized and } \\
\text { closed out in accordance with applicable MRPs. No operable } \\
\text { emergency sirens shall remain mounted on or powered from the } \\
\text { building. }\end{array}$ & & & \\
\hline $2 \mathrm{~A}$. & $\begin{array}{l}\text { RADIATION OCCURRENCE REPORTS, UNUSUAL OCCURRENCE REPORTS ANDJOR ANY } \\
\text { OTHER OUT-OF-STANDARD CONDITIONS REPORTS WILL BE FINALIZED AND } \\
\text { CLOSED BY WHC. }\end{array}$ & & & \\
\hline 3. & $\begin{array}{l}\text { The Fuel Supply Shutdown Facilities Interim Safety Basis Report and } \\
\text { Plant Emergency Procedures shall be reviewed and updated for } \\
\text { deactivation/shutdown status. }\end{array}$ & & & \\
\hline $3 A$. & $\begin{array}{l}\text { REVIEW AND UPDATE OF THE INTERIM SAFETY BASIS REPORT WILL BE } \\
\text { COMPLETED AND PROVIDED TO BHI } 6 \text { MONTHS PRIOR TO TURNOVER. }\end{array}$ & & & \\
\hline 3B. & $\begin{array}{l}\text { REVIEW AND UPDATE OF THE PLANT EMERGENCY PROCEDURES WILL BE } \\
\text { COMPLETED AND PROVIDED TO BHI } 6 \text { MONTHS PRIOR TO TURNOVER. }\end{array}$ & & & \\
\hline 4 A. & $\begin{array}{l}\text { CVI index, drawings, procedures, prints, photographs, history, } \\
\text { design and operating documents provided. } \\
\text { ANAILABLE ESSEFIIAL DRAWINGS, A DRAMING LIST, PROCEDURES, ETC. WILL } \\
\text { BE PROVIDED IN TURNOVER FILE. }\end{array}$ & & & \\
\hline 5. & $\begin{array}{l}\text { All permanent radiologically contaminated areas to be decontaminated } \\
\text { and released or the surface contamination levels reduced or fixed in } \\
\text { place to minimize re-suspension and \or migration of loose } \\
\text { contamination. }\end{array}$ & $\begin{array}{l}\text { NOT } \\
\text { APPLICABLE }\end{array}$ & & \\
\hline 6. & $\begin{array}{l}\text { Full compliance with Hanford Site Radiological Control Manual must } \\
\text { be assured, especially as it pertains to radiological post ing. } \\
\text { COMPLIANCE WITH HANFORD SITE RADIOLOGICAL CONTROL MANUAL WILL BE } \\
\text { CONFIRMED BY HAVING } 300 \text { AREA RADIOLOGICAL CONTROL PERFORM AN } \\
\text { ASSESSMENT AND WHC WILL CORRECT ALL NOTED DISCREPANCIES. }\end{array}$ & & & \\
\hline
\end{tabular}

6 


\begin{tabular}{|c|c|c|c|c|}
\hline & & $\begin{array}{l}379 \forall 9 I 7 d d Y \\
10 \mathrm{~N}\end{array}$ & 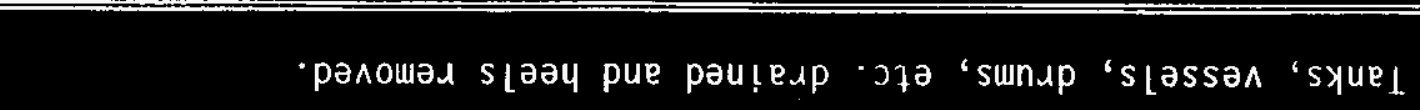 & $\cdot b l$ \\
\hline & & & 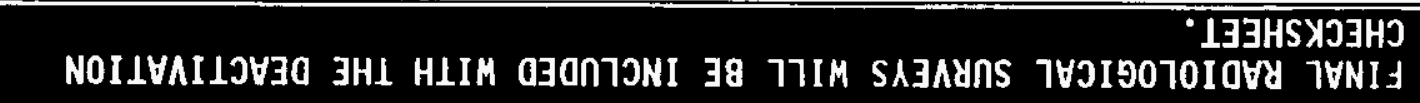 & •VEI \\
\hline & & & -alqel!ene skandns snfezs lej!bolo!ped [eu!j & $\bar{\varepsilon} \mathrm{EI}$ \\
\hline & & $\begin{array}{l}\text { 378 } 10 \mathrm{I} 7 \mathrm{ddV} \\
10 \mathrm{~N}\end{array}$ & 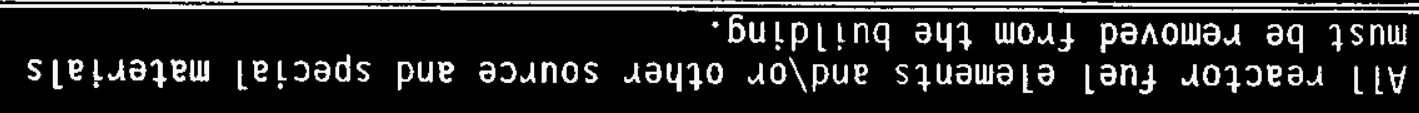 & $\cdot 2 I$ \\
\hline & & $\begin{array}{l}\text { 379V3I 7ddY } \\
\text { LON }\end{array}$ & 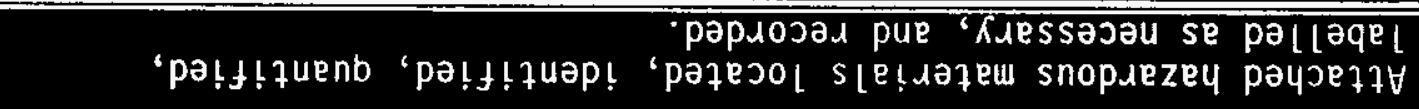 & $\cdot[\mathrm{I}$ \\
\hline & & $\begin{array}{l}\text { 378VJI Idd } \\
\text { LON }\end{array}$ & 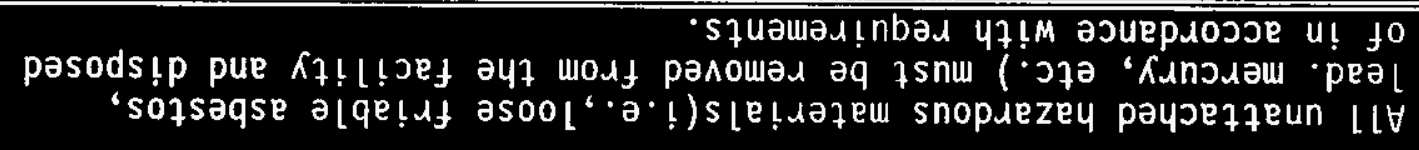 & $\cdot \mathrm{OI}$ \\
\hline & & & 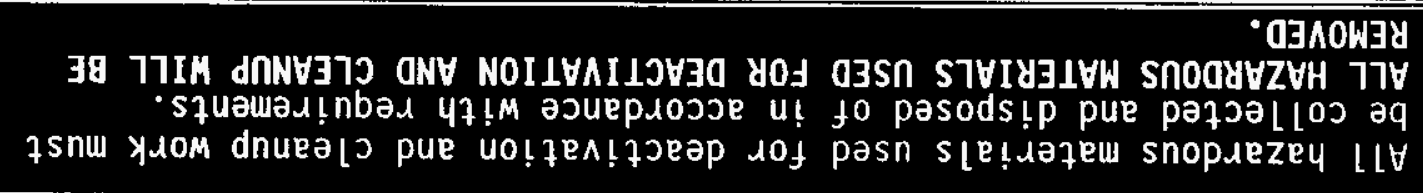 & $\begin{array}{l}\bullet 6 \\
: 6\end{array}$ \\
\hline & & & 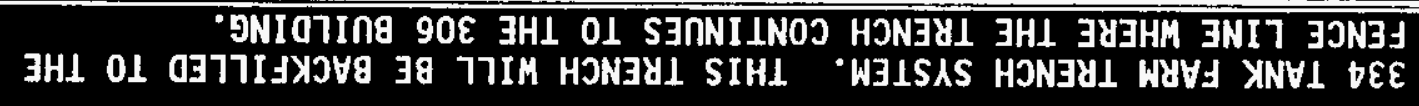 & $\cdot 08$ \\
\hline & & & 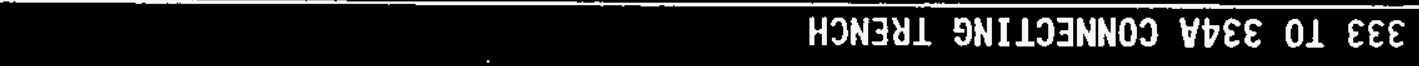 & $\cdot 98$ \\
\hline & & & 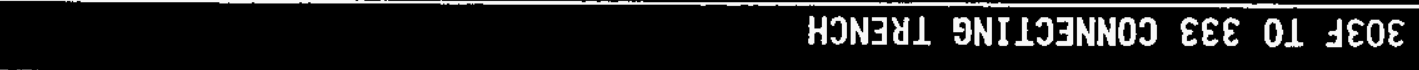 & $\cdot 88$ \\
\hline & & & 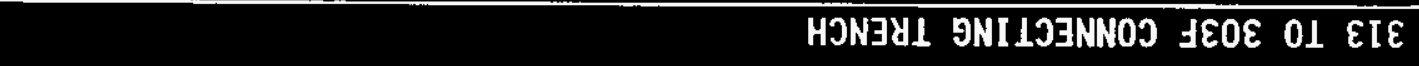 & $\cdot \mathrm{\cdot} 8$ \\
\hline & & & 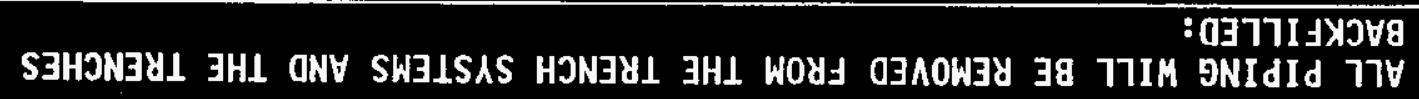 & \\
\hline & & & - panouad afsem pax!ul pue an!foeo!ped pajozs $I I \forall$ & $\cdot 8$ \\
\hline & & $\begin{array}{c}37949 I 7 d d y \\
\text { LON }\end{array}$ & 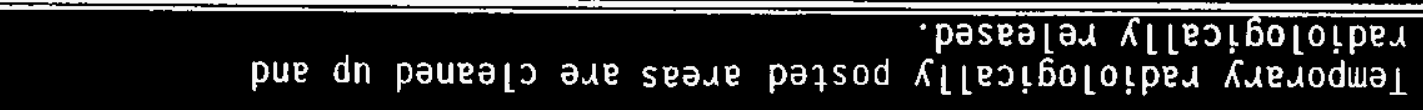 & $\cdot L$ \\
\hline $\begin{array}{c}1 \text { INJWnJ00 } \\
\text { NoI }\end{array}$ & IHg & ЭHM & & \\
\hline /NOIIVINJWJרdWI & \multicolumn{2}{|c|}{ 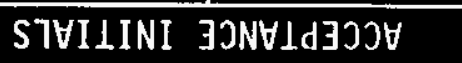 } & 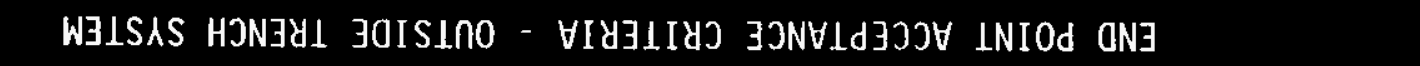 & $\#$ WJ1I \\
\hline
\end{tabular}




\begin{tabular}{|c|c|c|c|c|}
\hline \multicolumn{5}{|c|}{ END POINT TABLE } \\
\hline \multirow[t]{2}{*}{ ITEM \# } & \multirow[t]{2}{*}{ END POINT ACCEPTANCE CRITERIA - OUTSIDE TRENCH SYSTEM } & \multicolumn{2}{|c|}{ ACCEPTANCE INITIALS } & \multirow{2}{*}{$\begin{array}{l}\text { IMPLEMENTATION/ } \\
\text { VERIF ICATION } \\
\text { DOCUMENT }\end{array}$} \\
\hline & & WHC & BHI & \\
\hline 15. & $\begin{array}{l}\text { All elevator and crane systems are laid up with documentation of the } \\
\text { type, weight and class of fluid required for operation, should that } \\
\text { become desirable in the future. }\end{array}$ & $\begin{array}{l}\text { NOT } \\
\text { APPLICABLE }\end{array}$ & & \\
\hline 16. & $\begin{array}{l}\text { Documentation is available confirming that zero energy checks were } \\
\text { made on all de-energized electrical circuits and isolated } \\
\text { pressurized systems e.g., water, service air, steam, etc. using } \\
\text { existing documents to the extent possible. }\end{array}$ & $\begin{array}{l}\text { NOT } \\
\text { APPLICABLE }\end{array}$ & & \\
\hline 17 . & $\begin{array}{l}\text { Electrical systems reduced to that necessary for } S \& M \text { and subsequent } \\
\text { D\&D. This includes removal of emergency } 1 \text { ight fixtures and the } \\
\text { associated batteries. Where appropriate, centralize the remaining } \\
\text { electrical services to a single point. }\end{array}$ & $\begin{array}{l}\text { NOT } \\
\text { APPLICABLE }\end{array}$ & & \\
\hline 18. & $\begin{array}{l}\text { All heating, ventilation and air conditioning supply and exhaust air } \\
\text { systems shut down and de-energized. This criteria includes } \\
\text { evaluation and, as appropriate, the shutdown of the High-efficiency } \\
\text { particulate air-filtered (HEPA) ventilation exhaust from process } \\
\text { areas. }\end{array}$ & $\begin{array}{l}\text { NOT } \\
\text { APPLICABLE }\end{array}$ & & \\
\hline 19 . & All unneeded equipment shut down. & $\begin{array}{l}\text { NOT } \\
\text { APPLICABLE }\end{array}$ & & \\
\hline 20. & Fire protection systems downgraded. & $\begin{array}{l}\text { NOT } \\
\text { APPLICABLE }\end{array}$ & & \\
\hline 21. & $\begin{array}{l}\text { The radiation space monitoring system should be reduced to a level } \\
\text { required by the Health Physics organization. }\end{array}$ & $\begin{array}{l}\text { NOT } \\
\text { APPLICABLE }\end{array}$ & & \\
\hline 22. & $\begin{array}{l}\text { The cont inuous air monitoring system should be reduced to a level } \\
\text { required by the Health Physics organization. }\end{array}$ & $\begin{array}{l}\text { NOT } \\
\text { APPLICABLE }\end{array}$ & & \\
\hline 23. & The criticality monitoring and alarm system should be deactivated. & $\begin{array}{l}\text { NOT } \\
\text { APPLICABLE }\end{array}$ & & \\
\hline 24. & $\begin{array}{l}\text { Reduce or eliminate the electrical, service air, and water supply } \\
\text { services to the building in accordance with the requirements for } \\
\text { S\&M. }\end{array}$ & $\begin{array}{l}\text { NOT } \\
\text { APPLICABLE }\end{array}$ & & \\
\hline
\end{tabular}




\begin{tabular}{|c|c|c|c|c|}
\hline \multicolumn{5}{|c|}{ END POINT TABLE } \\
\hline \multirow[t]{2}{*}{ ITEM \# } & \multirow[t]{2}{*}{ END POINT ACCEPTANCE CRITERIA - OUTSIDE TRENCH SYSTEM } & \multicolumn{2}{|c|}{ ACCEPTANCE INITIALS } & \multirow{2}{*}{$\begin{array}{l}\text { IMPLEMENTATION/ } \\
\text { VERIFICATION } \\
\text { DOCUMENT }\end{array}$} \\
\hline & & WHC & BHI & \\
\hline 25. & Remove all emergency lighting and dispose of associated batteries. & $\begin{array}{l}\text { NOT } \\
\text { APPLICABLE }\end{array}$ & & \\
\hline 26. & The building steam system should be deactivated and tagged. & $\begin{array}{l}\text { NOT } \\
\text { APPLICABLE }\end{array}$ & & \\
\hline 27. & $\begin{array}{l}\text { All effluent flow routes to disposal sites are isolated by sealing } \\
\text { at the building. }\end{array}$ & & & \\
\hline $27 \mathrm{~A}$. & PROCESS SENER FLOH ROUTE WILL BE ISOLATED BY SEALING. & & & \\
\hline 28. & $\begin{array}{l}\text { All building penetrations, i.e., louvers, open piping, etc. shall be } \\
\text { closed off to prevent bird and animal intrusion. }\end{array}$ & $\begin{array}{l}\text { NOT } \\
\text { APPLICABLE }\end{array}$ & & \\
\hline 29. & $\begin{array}{l}\text { Al1 systems that were open to facilitate deactivation and could } \\
\text { present a radiological and/or an industrial safety problem if left } \\
\text { open must be adequately closed off. } \\
\text { ALL OPEN SYSTEMS PRESENTING RADIOLOGICAL AND/OR IWDUSTRIAL SAFETY } \\
\text { PROBLEM HILL BE ADEQUATELY CLOSED OFF. }\end{array}$ & & & \\
\hline 30. & Roof leaks repaired and suspect areas reviewed. & $\begin{array}{l}\text { NOT } \\
\text { APPLICABLE }\end{array}$ & & \\
\hline 31 . & Office furniture and supplies removed. & $\begin{array}{l}\text { NOT } \\
\text { APPLICABLE }\end{array}$ & & \\
\hline 32. & Unnecessary parts, tools, and portable equipment removed. & $\begin{array}{l}\text { NOT } \\
\text { APPLICABLE }\end{array}$ & & \\
\hline 33. & Janitorial supplies and unattached hazardous materials removed. & $\begin{array}{l}\text { NOT } \\
\text { APPLICABLE }\end{array}$ & & \\
\hline 34 . & Loose and \or damaged asbestos removed or stabilized. & $\begin{array}{l}\text { NOT } \\
\text { APPLICABLE }\end{array}$ & & \\
\hline 35 . & $\begin{array}{l}\text { Perform good housekeeping in all zones and areas in and around the } \\
\text { facility. }\end{array}$ & & & \\
\hline $35 A$. & $\begin{array}{l}\text { GOOD HOUSEKEEPING WILL BE PERFORHED IN ALL ZONES AND AREAS IN AND } \\
\text { AROUND FACILITY. }\end{array}$ & & & \\
\hline
\end{tabular}




\begin{tabular}{|c|c|c|c|c|}
\hline \multicolumn{5}{|c|}{ END POINT TABLE } \\
\hline \multirow[t]{2}{*}{ ITEM \# } & \multirow[t]{2}{*}{ END POINT ACCEPTANCE CRITERIA - OUTSIDE TRENCH SYSTEM } & \multicolumn{2}{|c|}{ ACCEPTANCE INITIALS } & \multirow{2}{*}{$\begin{array}{l}\text { IMPLEMENTATION/ } \\
\text { VERIFICATION } \\
\text { DOCUMENT }\end{array}$} \\
\hline & & WHC & BHI & \\
\hline 36. & $\begin{array}{l}\text { Any required Environmental Monitoring Systems maintained by the ERC } \\
\text { are identified and in serviceable condition. }\end{array}$ & $\begin{array}{l}\text { NOT } \\
\text { APPLICABLE }\end{array}$ & & \\
\hline 37. & $\begin{array}{l}\text { Any seal pits, dry or wet, must be identified and their radiological } \\
\text { status documented and of file. }\end{array}$ & $\begin{array}{c}\text { NOT } \\
\text { APPLICABLE }\end{array}$ & & \\
\hline 38. & $\begin{array}{l}\text { All doors should be bolted shut from the inside except those needed } \\
\text { for entrance or to comply with emergency egress requirements. } \\
\text { Emergency exit doors should have crashbar systems installed. Keys } \\
\text { needed for entrance shall be turned over to BHI. }\end{array}$ & $\begin{array}{l}\text { NOT } \\
\text { APPLICABLE }\end{array}$ & & \\
\hline 39. & $\begin{array}{l}\text { Environmental and/or other applicable permits associated with the } \\
\text { facility must be reviewed with EM-40/BHI to determine the status and } \\
\text { applicability for maintaining them. } \\
\text { CLEAN CLOSURE VERIFICATION OF THE WATS SYSTEM WILL BE PROVIDED. }\end{array}$ & $\begin{array}{l}\text { NOT } \\
\text { APPLICABLE }\end{array}$ & & \\
\hline 40. & $\begin{array}{l}\text { Deactivation of the facility shall be accomplished to the extent } \\
\text { that future surveillance inspections will not be required on a } \\
\text { frequency of more than once each quarter. }\end{array}$ & & & \\
\hline 40A. & $\begin{array}{l}\text { DEACTIVATION OF FACILITY WILL BE ACCOMPLISHED TO MINIMIZE } \\
\text { SURVEILLANCE. }\end{array}$ & & & \\
\hline & END POINTS ACCEPIABLE & & & \\
\hline & WHC & & & \\
\hline
\end{tabular}




\section{DISTRIBUTION SHEET}

\begin{tabular}{|c|c|c|c|c|c|}
\hline \multirow{2}{*}{$\begin{array}{l}\text { To } \\
\text { Distribution }\end{array}$} & \multirow{2}{*}{\multicolumn{3}{|c|}{$\begin{array}{l}\text { From } \\
\text { J. A. Remaize }\end{array}$}} & \multicolumn{2}{|c|}{ Page 1 of 1} \\
\hline & & & & \multicolumn{2}{|c|}{ Date $3 / 20 / 96$} \\
\hline \multirow{2}{*}{\multicolumn{4}{|c|}{$\begin{array}{l}\text { Project Title/Work Order } \\
\text { End Point Criteria for Fuel Supply Shutdown Facilities }\end{array}$}} & \multicolumn{2}{|c|}{ EDT No. 606213} \\
\hline & & & & \multicolumn{2}{|c|}{ ECN No. } \\
\hline Name & MSIN & $\begin{array}{c}\text { Text } \\
\text { With All } \\
\text { Attach. }\end{array}$ & Text Only & $\begin{array}{l}\text { Attach./ } \\
\text { Appendix } \\
\text { Only }\end{array}$ & $\begin{array}{l}\text { EDT/ECN } \\
\text { Only }\end{array}$ \\
\hline $\begin{array}{l}\text { Central Files foriginat-2t- } \\
\text { A. M. Horner } \\
\text { I. L. Metcalf (5) } \\
\text { D. E. Rasmussen } \\
\text { J. A. Remaize } \\
\text { M. E. Riste } \\
\end{array}$ & $\begin{array}{r}A 3-89 \\
48-04 \\
L 6-57 \\
L 6-26 \\
N 1-47 \\
L 6-26 \\
N 2-11 \\
\end{array}$ & $\begin{array}{l}X \\
X \\
X \\
X \\
X \\
X \\
\end{array}$ & & & \\
\hline S.P. Hansen & $L 6-2$ & & & & $x$ \\
\hline
\end{tabular}

* ADVANCED 